\title{
Computationally Sound Mechanized Proofs of Correspondence Assertions
}

\author{
Bruno Blanchet \\ Inria, Paris \\ Bruno.Blancheteinria.fr
}

\begin{abstract}
We present a new mechanized prover for showing correspondence assertions for cryptographic protocols in the computational model. Correspondence assertions are useful in particular for establishing authentication. Our technique produces proofs by sequences of games, as standard in cryptography. These proofs are valid for a number of sessions polynomial in the security parameter, in the presence of an active adversary. Our technique can handle a wide variety of cryptographic primitives, including shared-and public-key encryption, signatures, message authentication codes, and hash functions. It has been implemented in the tool CryptoVerif and successfully tested on examples from the literature.
\end{abstract}

\section{Introduction}

Correspondence assertions on cryptographic protocols are properties of the form "if some events have been executed, then some other events have been executed", where each event corresponds to a certain point in the protocol, possibly with arguments. An event can be formalized by a special instruction event $e\left(M_{1}, \ldots, M_{m}\right)$, which simply records that the event $e\left(M_{1}, \ldots, M_{m}\right)$ has been executed. Woo and Lam [63] introduced correspondence assertions to express the authentication properties of cryptographic protocols, such as "if $B$ terminates a run of the protocol, apparently with $A$, then $A$ has started a run of the protocol, apparently with $B$." This property can be written more formally "if event $\operatorname{Bterminates}(A)$ has been executed, then event $A \operatorname{starts}(B)$ has been executed", where event Bterminates $(X)$ occurs at the point where $B$ terminates a run and he thinks he talks to $X$, and event $\operatorname{Astarts}(Y)$ occurs at the point where $A$ starts a run with $Y$. Correspondence assertions have become a standard tool for reasoning on cryptographic protocols.

The main novelty of our work lies in the model in which we prove correspondence assertions. Indeed, there are two main models for cryptographic protocols. In the compu- tational model, cryptographic primitives are functions on bitstrings and the adversary is a polynomial-time probabilistic Turing machine. In this realistic model, proofs are usually manual. In the formal, Dolev-Yao model, cryptographic primitives are considered as perfect blackboxes represented by function symbols, and the adversary is restricted to compute with these blackboxes. There already exist several techniques for proving correspondence assertions automatically in this abstract model, e.g. $[18,36]$. However, in general, these proofs are not sound with respect to the computational model.

Since the seminal paper by Abadi and Rogaway [6], there has been much interest in relating both models [4, $11,14,30,31,38,39,50,51]$, to show the soundness of the Dolev-Yao model with respect to the computational model, and thus obtain automatic proofs of protocols in the computational model. However, this approach has limitations: since the computational and Dolev-Yao models do not correspond exactly, additional hypotheses are necessary in order to guarantee soundness. (For example, for symmetric encryption, key cycles have to be excluded, or a specific security definition of encryption is needed [8].)

In this paper, we adopt a different approach: our tool proves correspondences directly in the computational model. In order to achieve such proofs, we extend our previous approach for secrecy [20,21]. We produce proofs by sequences of games, as used by cryptographers [17, 57-59]: the initial game represents the protocol, for which we want to prove that the probability of breaking a certain correspondence is negligible; intermediate games are obtained each from the previous one by transformations such that the difference of probability between consecutive games is negligible; the final game is such that the desired probability can directly be shown to be negligible from the form of the game. The desired probability is then negligible in the initial game.

In order to extend our approach to correspondence assertions, we slightly extend the calculus that we use to represent games, so that it can specify events. The game transformations that we used for secrecy can also be used for correspondences, without change. However, we still need 
to check that the correspondence holds on the final game. So, we introduce a rich language of correspondence assertions, and show how to check them automatically. This language allows one to specify both injective correspondences (if some event has been executed $n$ times, then some other events have been executed at least $n$ times) and noninjective correspondences (if some events have been executed, then some other events have been executed at least once), as well as properties of the form "if some events have been executed, then some formula holds".

Moreover, we also show how to use correspondences in order to prove mutual authentication and authenticated key exchange. Mutual authentication is an immediate consequence of correspondences. The situation is more subtle for authenticated key exchange: intuitively, we need to prove the secrecy of the key. Since the key is shared between two participants of the protocol, the secrecy of the key is not simply the secrecy of a single variable, as we could prove in $[20,21]$. However, we show that by combining correspondences with the secrecy of the variable that contains the key for one of the participants of the protocol, we can prove the standard notion of authenticated key exchange.

The prover succeeds in a fully automatic way for many examples. For delicate cases, our prover allows the user to indicate the main game transformations to perform, such as applying the security of a certain cryptographic primitive for a certain secret key. Importantly, the prover is always sound, whatever indications the user gives.

The verification of correspondences has been implemented in our prover CryptoVerif (19200 lines of Ocaml for version 1.06 of CryptoVerif), available at http:// cryptoverif.inria.fr.

Related Work Results that show the soundness of the Dolev-Yao model with respect to the computational model, e.g. $[31,39,51]$, make it possible to use Dolev-Yao provers in order to prove correspondences in the computational model. In particular, a tool [29] has been built based on [31] in order to make computational proofs using the Dolev-Yao prover AVISPA, for protocols that use public-key encryption and signatures. However, computational soundness results have limitations, in particular in terms of allowed cryptographic primitives (they must satisfy strong security properties so that they correspond to Dolev-Yao style primitives), and they require some restrictions on protocols (such as the absence of key cycles).

Several frameworks exist for formalizing proofs of protocols in the computational model. Backes, Pfitzmann, and Waidner [10-12] have designed an abstract cryptographic library including symmetric and public-key encryption, message authentication codes, signatures, and nonces and shown its soundness with respect to computational primitives, under arbitrary active attacks. This framework shares some limitations with the computational soundness results, for instance the exclusion of key cycles and the fact that symmetric encryption has to be authenticated. It relates the computational model to a non-standard version of the Dolev-Yao model, in which the length of messages is still present. It has been used for a computationally-sound machine-checked proof of the Needham-Schroeder-Lowe protocol [60].

Canetti and Herzog [26] show how a Dolev-Yao-style symbolic analysis can be used to prove security properties of protocols (including authentication) within the framework of universal composability [24], for a restricted class of protocols using public-key encryption as only cryptographic primitive. Then, they use the automatic Dolev-Yao verification tool ProVerif [19] for verifying protocols in this framework.

Canetti et al. [25] use the framework of timebounded task-PIOAs (Probabilistic Input/Output Automata) for proving cryptographic protocols in the computational model. This framework allows them to combine probabilistic and non-deterministic behaviors.

Lincoln et al. [46, 47, 49, 52, 56] developed a probabilistic polynomial-time calculus for the analysis of security protocols. This calculus comes with a notion of process equivalence, used in particular to prove authentication properties in [47]. This calculus resembles ours in that both are probabilistic polynomial-time variants of the pi calculus. (The restriction chooses a fresh random number. The replication is polynomially bounded.) However, it differs from our calculus since it uses an explicit probabilistic scheduler while, in our calculus, the adversary schedules the processes. Our calculus also adds arrays in order to store all values of variables, which is key to our proofs, as we shall see below.

Datta et al. [32,33] have designed a computationally sound logic that enables them to prove computational security properties using a logical deduction system.

Corin and Hartog [28] use a probabilistic Hoare-style logic for formalizing game-based cryptographic proofs.

All these frameworks can be used to prove security properties of protocols in the computational sense, but except for [26] which relies on a Dolev-Yao prover and for the machine-checked proofs of [60], they have not been mechanized up to now, as far as we know.

Other works provide proofs in the computational model, but only for secrecy. Laud [43] designed an automatic analysis for protocols using shared-key encryption, with passive adversaries. He extended it to active adversaries, but with only one session of the protocol [44]. The type system of $[9,45]$ handles shared-key and public-key encryption, with an unbounded number of sessions. This system relies on the Backes-Pfitzmann-Waidner library.

Barthe, Cerderquist, and Tarento [13,61] have formal- 
ized the generic model and the random oracle model in the interactive theorem prover Coq, and proved signature schemes in this framework. In contrast to our specialized prover, proofs in generic interactive theorem provers require a lot of human effort, to build a detailed enough proof for the theorem prover to check it.

Halevi [37] explains that implementing an automatic prover based on sequences of games would be useful, and suggests ideas in this direction, but does not actually implement one.

Outline The next section recalls the process calculus that we use to represent games and extends it with events. Section 3 defines the correspondence assertions that we prove. Section 4 recalls the definition of observational equivalence and extends it with events. Section 5 illustrates on an example the game transformations used in our proofs. Section 6 details how we prove correspondences. Section 7 shows how to prove standard notions of authentication and authenticated key exchange using correspondences. Finally, Section 8 summarizes our experimental results and Section 9 concludes. The appendix contains details on the semantics of the calculus, the proof engine we use for reasoning on games, the proofs of our results, and our experiments.

\section{A Calculus for Games}

In this section, we review the process calculus defined in $[20,21]$ in order to model games used in computational security proofs. This calculus has been carefully designed to make the automatic proof of cryptographic protocols easier. We extend this calculus with parametric events, which serve in the definition of correspondences.

We illustrate this calculus on the following example, inspired by the corrected Woo-Lam public key protocol [64]:

$$
\begin{aligned}
& B \rightarrow A:(N, B) \\
& A \rightarrow B:\left\{p k_{A}, B, N\right\}_{s k_{A}}
\end{aligned}
$$

This protocol is a simple nonce challenge: $B$ sends to $A$ a fresh nonce $N$ and its identity. $A$ replies by signing the nonce $N, B$ 's identity, and $A$ 's public key (which we use here instead of $A$ 's identity for simplicity: this avoids having to relate identities and keys; the prover can obviously also handle the version with $A$ 's identity). The signatures are assumed to be (existentially) unforgeable under chosen message attacks (UF-CMA) [35], so, when $B$ receives the signature, $B$ is convinced that $A$ is present. The signature cannot be a replay because the nonce $N$ is signed.

In our calculus, this protocol is encoded by the following process $G_{0}$, explained below:

$$
\begin{aligned}
& G_{0}= c_{0}() ; \text { new } r k_{A}: \text { keyseed; let } p k_{A}=\operatorname{pkgen}\left(r k_{A}\right) \text { in } \\
& \text { let } s k_{A}=\operatorname{skgen}\left(r k_{A}\right) \text { in } \overline{c_{1}}\left\langle p k_{A}\right\rangle ;\left(Q_{A} \mid Q_{B}\right)
\end{aligned}
$$

$$
\begin{aligned}
Q_{A}= & !^{i_{A} \leq n} c_{2}\left[i_{A}\right]\left(x_{N}: \text { nonce, } x_{B}: \text { host }\right) ; \\
& \text { event } e_{A}\left(p k_{A}, x_{B}, x_{N}\right) ; \text { new } r: \text { seed; } \\
& \overline{c_{3}\left[i_{A}\right]}\left\langle\operatorname{sign}\left(\operatorname{concat}\left(p k_{A}, x_{B}, x_{N}\right), \text { sk } k_{A}, r\right)\right\rangle \\
Q_{B}= & !^{i_{B} \leq n} c_{4}\left[i_{B}\right]\left(x_{p k_{A}}: \text { pkey }\right) ; \text { new } N: \text { nonce; } \\
& \overline{c_{5}\left[i_{B}\right]}\langle N, B\rangle ; c_{6}\left[i_{B}\right](s: \text { signature }) ; \\
& \text { if verify }\left(\operatorname{concat}\left(x_{p k_{A}}, B, N\right), x_{p k_{A}}, s\right) \text { then } \\
& \text { if } x_{p k_{A}}=p k_{A} \text { then event } e_{B}\left(x_{p k_{A}}, B, N\right)
\end{aligned}
$$

The process $G_{0}$ is assumed to run in interaction with an adversary, which also models the network. $G_{0}$ first receives an empty message on channel $c_{0}$, sent by the adversary. Then, it chooses randomly with uniform probability a bitstring $r k_{A}$ in the type keyseed, by the construct new $r k_{A}$ : keyseed. A type $T$, such as keyseed, aims at denoting a set of bitstrings. However, the considered set of bitstrings depends on the security parameter $\eta$, which determines the length of keys. So, more precisely, a type $T$ corresponds for each value of $\eta$ to a set of bitstrings denoted by $I_{\eta}(T)$. Then, $G_{0}$ generates the public key $p k_{A}$ corresponding to the coins $r k_{A}$, by calling the public-key generation algorithm pkgen. Similarly, $G_{0}$ generates the secret key $s k_{A}$ by calling skgen. It outputs the public key $p k_{A}$ on channel $c_{1}$, so that the adversary has this public key.

After outputting this message, the control passes to the receiving process, which is part of the adversary. Several processes are then made available, which represent the roles of $A$ and $B$ in the protocol: the process $Q_{A} \mid Q_{B}$ is the parallel composition of $Q_{A}$ and $Q_{B}$; it makes simultaneously available the processes defined in $Q_{A}$ and $Q_{B}$. Let $Q_{A}^{\prime}$ and $Q_{B}^{\prime}$ be such that $Q_{A}=!^{i_{A} \leq n} Q_{A}^{\prime}$ and $Q_{B}=!^{i_{B} \leq n} Q_{B}^{\prime}$. The replication $!^{i_{A}}{ }^{\leq n} Q_{A}^{\prime}$ represents $n$ copies of the process $Q_{A}^{\prime}$, indexed by the replication index $i_{A}$. (The symbol $n$ corresponds to an integer $I_{\eta}(n)$ for each value of the security parameter $\eta ; I_{\eta}(n)$ is required to be a polynomially bounded function of $\eta$.) The process $Q_{A}^{\prime}$ begins with an input on channel $c_{2}\left[i_{A}\right]$; the channel is indexed with $i_{A}$ so that the adversary can choose which copy of the process $Q_{A}^{\prime}$ receives the message by sending it on channel $c_{2}\left[i_{A}\right]$ for the appropriate value of $i_{A}$. The situation is similar for $Q_{B}^{\prime}$, which expects a message on channel $c_{4}\left[i_{B}\right]$. The adversary can then run each copy of $Q_{A}^{\prime}$ or $Q_{B}^{\prime}$ simply by sending a message on the appropriate channel $c_{2}\left[i_{A}\right]$ or $c_{4}\left[i_{B}\right]$.

The process $Q_{B}^{\prime}$ first expects on channel $c_{4}\left[i_{B}\right]$ a message $x_{p k_{A}}$ in the type pkey of public keys. This message is not really part of the protocol. It serves for starting a new session of the protocol, in which $B$ interacts with the participant of public key $x_{p k_{A}}$. For starting a session between $A$ and $B$, this message should be $p k_{A}$. Then, $Q_{B}^{\prime}$ chooses randomly with uniform probability a nonce $N$ in the type nonce. The type nonce is large: a type $T$ is large when the inverse of its cardinal $\frac{1}{\left|I_{\eta}(T)\right|}$ is negligible, so that collisions between independent random numbers chosen uniformly in 
a large type have negligible probability. (The probability $f(\eta)$ is negligible when for all polynomials $q$, there exists $\eta_{o} \in \mathbb{N}$ such that for all $\eta>\eta_{0}, f(\eta) \leq \frac{1}{q(\eta)}$. The probability $f(\eta)$ is overwhelming when $1-f(\eta)$ is negligible.) $Q_{B}^{\prime}$ sends the message $(N, B)$ on channel $c_{5}\left[i_{B}\right]$. The control then passes to the receiving process, included in the adversary. This process is expected to forward this message $(N, B)$ on channel $c_{2}\left[i_{A}\right]$, but may proceed differently in order to mount an attack against the protocol.

Upon receiving a message $\left(x_{N}, x_{B}\right)$ on channel $c_{2}\left[i_{A}\right]$, where the bitstring $x_{N}$ is in the type nonce and $x_{B}$ in the type host, the process $Q_{A}^{\prime}$ executes the event $e_{A}\left(p k_{A}, x_{B}\right.$, $\left.x_{N}\right)$. This event does not change the state of the system. Events just record that a certain program point has been reached, with certain values of the arguments of the event. Then, $Q_{A}^{\prime}$ chooses randomly with uniform probability a bitstring $r$ in the type seed; this random bitstring is next used as coins for the signature algorithm. Finally, $Q_{A}^{\prime}$ outputs the signed message $\left\{p k_{A}, x_{B}, x_{N}\right\}_{s k_{A}}$. (The function concat concatenates its arguments, with information on the length of these arguments, so that the arguments can be recovered from the concatenation.) The control then passes to the receiving process, which should forward this message on channel $c_{6}\left[i_{B}\right]$ if it wishes to run the protocol correctly.

Upon receiving a message $s$ on $c_{6}\left[i_{B}\right], Q_{B}^{\prime}$ verifies that the signature $s$ is correct and, if $x_{p k_{A}}=p k_{A}$, that is, if $B$ runs a session with $A$, it executes the event $e_{B}\left(x_{p k_{A}}, B, N\right)$. Our goal is to prove that, if event $e_{B}$ is executed, then event $e_{A}$ has also been executed. However, when $B$ runs a session with a participant other than $A$, it is perfectly correct that $B$ terminates without event $e_{A}$ being executed; that is why event $e_{B}$ is executed only when $B$ runs a session with $A$.

In our calculus, all variables defined under a replication are implicitly arrays. For example, the variable $x_{N}$ defined under $!^{i_{A}} \leq n$ is implicitly an array indexed by the replication index $i_{A}: x_{N}$ is an abbreviation for $x_{N}\left[i_{A}\right]$. Similarly, $x_{B}$ is an abbreviation for $x_{B}\left[i_{A}\right], r$ for $r\left[i_{A}\right], x_{p k_{A}}$ for $x_{p k_{A}}\left[i_{B}\right]$, $N$ for $N\left[i_{B}\right]$, and $s$ for $s\left[i_{B}\right]$. Using arrays allows us to remember the values of the variables in each copy of the processes, so that the whole state of the system is available. In our calculus, arrays replace lists often used by cryptographers in their proofs. For example, during the proof, all messages signed under $s k_{A}$ would be stored in a list, and by the unforgeability of signatures, when the verification of the signature of a message succeeds, we would be sure that this message occurs in the list. In our calculus, we will store messages in arrays instead. Arrays come with a lookup construct: find $u_{1} \leq n_{1}, \ldots, u_{m} \leq n_{m}$ suchthat defined $\left(M_{1}\right.$, $\left.\ldots, M_{l}\right) \wedge M$ then $P$ else $P^{\prime}$ looks for indices $u_{1}, \ldots, u_{m}$ such that $M_{1}, \ldots, M_{l}$ are defined and $M$ is true. When such indices are found, it executes $P$; otherwise, it executes $P^{\prime}$. When several values of indices are possible, each possible value is chosen with the same probability. For example, find $u \leq n$ suchthat defined $\left(x_{N}[u]\right) \wedge x_{N}[u]=N$ then $P$ looks for an index $u$ such that $x_{N}[u]$ is defined and equal to $N$. Here, the find construct does not occur in the initial game, but will be introduced by game transformations.

As detailed in [20,21], we require some well-formedness invariants to guarantee that bitstrings are of their expected type and that arrays are used properly (that each cell of an array is assigned at most once during execution and that variables are accessed only after being initialized).

All processes of our calculus run in probabilistic polynomial time. The semantics of the calculus is defined by a probabilistic reduction relation on semantic configurations $\mathbb{C}$. We denote by $\operatorname{init} \operatorname{Config}(Q)$ the initial configuration associated to process $Q$. We refer the reader to Appendix A and [20] for additional details on this calculus and its semantics. Given a mapping $\rho$ from variable names to bitstrings, we write $\rho, M \Downarrow a$ when the term $M$ (built from function symbols and variables, without array accesses) evaluates to bitstring $a$. We denote by $\mathcal{E}$ a sequence of events of the form $e\left(a_{1}, \ldots, a_{n}\right)$, where $e$ is an event symbol and $a_{1}, \ldots, a_{n}$ are bitstrings. We denote by $\operatorname{Pr}[\exists(\mathbb{C}, \mathcal{E})$, initConfig $(Q) \stackrel{\mathcal{E}}{\rightarrow} \mathbb{C} \wedge \phi(\mathbb{C}, \mathcal{E})]$ the probability that there exists a sequence of events $\mathcal{E}$ and a semantic configuration $\mathbb{C}$ such that $Q$ reduces to $\mathbb{C}$, executing events $\mathcal{E}$ on the trace, and the logical formula $\phi(\mathbb{C}, \mathcal{E})$ holds. We introduce an additional polynomialtime algorithm, a distinguisher $D$ that takes as input a sequence of events and returns true or false. An example of distinguisher is $D_{e}$ defined by $D_{e}(\mathcal{E})=$ true if and only if $e \in \mathcal{E}$ : this distinguisher detects the execution of event $e$. Given a distinguisher $D$, we denote by $\operatorname{Pr}[Q: D]=\operatorname{Pr}[\exists(\mathbb{C}, \mathcal{E})$, initConfig $(Q) \stackrel{\mathcal{E}}{\rightarrow} \mathbb{C} \wedge D(\mathcal{E}) \wedge$ $\mathbb{C}$ does not reduce] the probability that the process $Q$ executes a sequence of events $\mathcal{E}$ such that $D(\mathcal{E})=$ true. These probabilities depend on the security parameter $\eta$; we omit it to lighten notations.

We use an evaluation context $C$ to represent the adversary. An evaluation context is a process with a hole, of one of the following forms: a hole [], a process in parallel with an evaluation context $Q \mid C$, or a restriction newChannel $c ; C$, which limits the scope of the channel $c$ to the context $C$. We denote by $C[Q]$ the process obtained by replacing the hole of $C$ with $Q$. When $V$ is a set of variables defined in $Q$, an evaluation context $C$ is said to be acceptable for $Q$ with public variables $V$ if and only if the common variables of $C$ and $Q$ are in $V$, and $C[Q]$ satisfies the well-formedness invariants. The set $V$ contains the variables the context is allowed to access (using find).

When $P$ is under replications $!^{i_{1} \leq n_{1}} \ldots !^{i_{m} \leq n_{m}}$, we say that the replication indices at $P$ are $i_{1}, \ldots, i_{m}$. We denote by $\widetilde{i}$ a sequence of replication indices $i_{1}, \ldots, i_{m}$, and by $\widetilde{M}$ a sequence of terms $M_{1}, \ldots, M_{m}$. We denote by fc $(P)$ the set of free channels of $P$, and by $\operatorname{var}(P)$ the set of variables 
that occur in $P$. We also use the notation $\operatorname{var}(\cdot)$ for contexts, terms, and formulas.

\section{Definition of Correspondences}

In this section, we define non-injective and injective correspondences.

\subsection{Non-injective Correspondences}

A non-injective correspondence is a property of the form "if some events have been executed, then some other events have been executed at least once". Here, we generalize these correspondences to implications between logical formulae $\psi \Rightarrow \phi$, which may contain events. We use the following logical formulae:

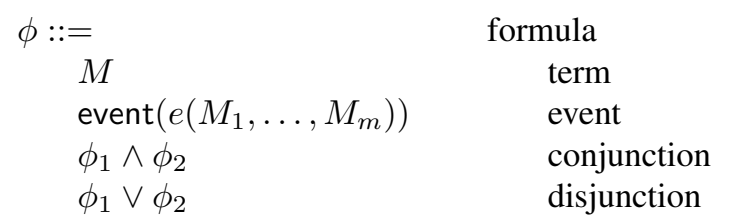

Terms $M, M_{1}, \ldots, M_{m}$ in formulae must not contain array accesses, and their variables are assumed to be distinct from variables of processes. The formula $M$ holds when $M$ evaluates to true. The formula event $\left(e\left(M_{1}, \ldots, M_{n}\right)\right)$ holds when the event $e\left(M_{1}, \ldots, M_{n}\right)$ has been executed. The conjunction and disjunction are defined as usual. More formally, we write $\rho, \mathcal{E} \vdash \phi$ when the sequence of events $\mathcal{E}$ satisfies the formula $\phi$, in the environment $\rho$ that maps variables to bitstrings. We define $\rho, \mathcal{E} \vdash \phi$ as follows:

$\rho, \mathcal{E} \vdash M$ if and only if $\rho, M \Downarrow$ true

$\rho, \mathcal{E} \vdash \operatorname{event}\left(e\left(M_{1}, \ldots, M_{m}\right)\right)$ if and only if

for all $j \leq m, \rho, M_{j} \Downarrow a_{j}$ and $e\left(a_{1}, \ldots, a_{m}\right) \in \mathcal{E}$

$\rho, \mathcal{E} \vdash \phi_{1} \wedge \phi_{2}$ if and only if $\rho, \mathcal{E} \vdash \phi_{1}$ and $\rho, \mathcal{E} \vdash \phi_{2}$

$\rho, \mathcal{E} \vdash \phi_{1} \vee \phi_{2}$ if and only if $\rho, \mathcal{E} \vdash \phi_{1}$ or $\rho, \mathcal{E} \vdash \phi_{2}$

Formulae denoted by $\psi$ are conjunctions of events.

Definition 1 The sequence of events $\mathcal{E}$ satisfies the correspondence $\psi \Rightarrow \phi$, written $\mathcal{E} \vdash \psi \Rightarrow \phi$, if and only if for all $\rho$ defined on $\operatorname{var}(\psi)$ such that $\rho, \mathcal{E} \vdash \psi$, there exists an extension $\rho^{\prime}$ of $\rho$ to $\operatorname{var}(\phi)$ such that $\rho^{\prime}, \mathcal{E} \vdash \phi$.

Intuitively, a sequence of events $\mathcal{E}$ satisfies $\psi \Rightarrow \phi$ when, if $\mathcal{E}$ satisfies $\psi$, then $\mathcal{E}$ satisfies $\phi$. The variables of $\psi$ are universally quantified; those of $\phi$ that do not occur in $\psi$ are existentially quantified.

Definition 2 We define a distinguisher $D$ such that $D(\mathcal{E})=$ true if and only if $\mathcal{E} \vdash \psi \Rightarrow \phi$. We denote this distinguisher $D$ simply by $\psi \Rightarrow \phi$ and write $\neg(\psi \Rightarrow \phi)$ for its negation.
The process $Q$ satisfies the correspondence $\psi \Rightarrow \phi$ with public variables $V$ if and only if for all evaluation contexts $C$ acceptable for $Q$ with public variables $V$ that do not contain events used by $\psi \Rightarrow \phi, \operatorname{Pr}[C[Q]: \neg(\psi \Rightarrow \phi)]$ is negligible.

A process satisfies $\psi \Rightarrow \phi$ when the probability that it generates a sequence of events $\mathcal{E}$ that does not satisfy $\psi \Rightarrow$ $\phi$ is negligible, in the presence of an adversary represented by the context $C$.

Example 1 Referring to the example $G_{0}$ of Section 2, the correspondence

$$
\operatorname{event}\left(e_{B}(x, y, z)\right) \Rightarrow \operatorname{event}\left(e_{A}(x, y, z)\right)
$$

means that, with overwhelming probability, for all $x, y, z$, if $e_{B}(x, y, z)$ has been executed, then $e_{A}(x, y, z)$ has been executed.

The correspondence

$$
\begin{aligned}
& \operatorname{event}\left(e_{1}(x)\right) \wedge \operatorname{event}\left(e_{2}(x)\right) \Rightarrow \\
& \quad \operatorname{event}\left(e_{3}(x)\right) \vee\left(\operatorname{event}\left(e_{4}(x, y)\right) \wedge \operatorname{event}\left(e_{5}(y, z)\right)\right)
\end{aligned}
$$

means that, with overwhelming probability, for all $x$, if $e_{1}(x)$ and $e_{2}(x)$ have been executed, then $e_{3}(x)$ has been executed or there exists $y$ such that both $e_{4}(x, y)$ and $e_{5}(x, y)$ have been executed.

\subsection{Injective Correspondences}

Injective correspondences are properties of the form "if some event has been executed $n$ times, then some other events have been executed at least $n$ times". In order to model them in our logical formulae, we extend the grammar of formulae $\phi$ with injective events inj-event $\left(e\left(M_{1}, \ldots, M_{m}\right)\right)$. The formula $\psi$ is a conjunction of (injective or non-injective) events. The conditions on the number of executions of events apply only to injective events.

The definition of formula satisfaction is also extended: we indicate at which step each injective event has been executed, by a "pseudo-formula" $\phi^{\tau}$ obtained from the formula $\phi$ by replacing terms and non-injective events with $\perp$ and injective events with the step $\tau$ at which they have been executed (that is, their index $\tau$ in the sequence of events $\mathcal{E}$ ) or $\perp$ when their execution is not required. For example, if $\phi=$ inj-event $\left(e_{1}(x)\right) \wedge\left(\operatorname{inj}\right.$-event $\left(e_{2}(x)\right) \vee$ inj-event $\left.\left(e_{3}(x)\right)\right)$, then $\phi^{\tau}$ is of the form $\tau_{1} \wedge\left(\tau_{2} \vee \tau_{3}\right)$ where $\tau_{1}$ is the execution step of $e_{1}(x)$ and either $\tau_{2}$ is the execution step of $e_{2}(x)$ or $\tau_{3}$ is the execution step of $e_{3}(x)$. (One of the steps $\tau_{2}$ and $\tau_{3}$ may be $\perp$, but not both.) We define formula satisfaction $\rho, \mathcal{E} \vdash^{\phi^{\tau}} \phi$ as follows: 
$\rho, \mathcal{E} \vdash^{\perp} M$ if and only if $\rho, M \Downarrow$ true

$\rho, \mathcal{E} \vdash^{\perp} \operatorname{event}\left(e\left(M_{1}, \ldots, M_{m}\right)\right)$ if and only if

for all $j \leq m, \rho, M_{j} \Downarrow a_{j}$ and $e\left(a_{1}, \ldots, a_{m}\right) \in \mathcal{E}$

$\rho, \mathcal{E} \vdash^{\tau}$ inj-event $\left(e\left(M_{1}, \ldots, M_{m}\right)\right)$ if and only if $\tau \neq \perp$,

for all $j \leq m, \rho, M_{j} \Downarrow a_{j}$, and $e\left(a_{1}, \ldots, a_{m}\right)=\mathcal{E}(\tau)$

$\rho, \mathcal{E} \vdash \phi_{1}^{\tau} \wedge \phi_{2}^{\tau} \phi_{1} \wedge \phi_{2}$ if and only if

$\rho, \mathcal{E} \vdash \phi_{1}^{\tau} \phi_{1}$ and $\rho, \mathcal{E} \vdash \phi_{2}^{\tau} \phi_{2}$

$\rho, \mathcal{E} \vdash \phi_{1}^{\tau} \vee \phi_{2}^{\tau} \phi_{1} \vee \phi_{2}$ if and only if

$\rho, \mathcal{E} \vdash^{\phi_{1}^{\tau}} \phi_{1}$ or $\rho, \mathcal{E} \vdash^{\phi_{2}^{\tau}} \phi_{2}$

This definition differs from the case of non-injective correspondences in that we propagate the pseudo-formula $\phi^{\tau}$ and, in the case of injective events, we make sure that the event has been executed at step $\tau$ by requiring that $\tau \neq \perp$ and $e\left(a_{1}, \ldots, a_{m}\right)=\mathcal{E}(\tau)$.

Given a function $\mathbb{F}$ that maps $\psi^{\tau}$ to $\phi^{\tau}$, the projection $f$ of $\mathbb{F}$ to the leaf at occurrence $o$ of $\phi$ is such that $f\left(\psi^{\tau}\right)$ is the leaf at occurrence $o$ of $\mathbb{F}\left(\psi^{\tau}\right)$. For example, if $\mathbb{F}$ maps $\psi^{\tau}$ to $\phi^{\tau}$ of the form $\tau_{1} \wedge\left(\tau_{2} \vee \tau_{3}\right)$, then $\mathbb{F}$ has three projections, which map $\psi^{\tau}$ to $\tau_{1}, \tau_{2}$, and $\tau_{3}$ respectively. We say that $\mathbb{F}$ is component-wise injective when each projection $f$ of $\mathbb{F}$ is such that $f\left(\psi_{1}^{\tau}\right)=f\left(\psi_{2}^{\tau}\right) \neq \perp$ implies $\psi_{1}^{\tau}=\psi_{2}^{\tau}$. (Ignoring the result $\perp, f$ is injective.)

Definition 3 The sequence of events $\mathcal{E}$ satisfies the correspondence $\psi \Rightarrow \phi$, written $\mathcal{E} \vdash \psi \Rightarrow \phi$, if and only if there exists a component-wise injective $\mathbb{F}$ such that for all $\rho$ defined on $\operatorname{var}(\psi)$, for all $\psi^{\tau}$ such that $\rho, \mathcal{E} \vdash^{\tau} \psi$, there exists an extension $\rho^{\prime}$ of $\rho$ to $\operatorname{var}(\phi)$ such that $\rho^{\prime}, \mathcal{E} \vdash^{\mathbb{F}\left(\psi^{\tau}\right)} \phi$.

Intuitively, a sequence of events $\mathcal{E}$ satisfies $\psi \Rightarrow \phi$ when, if $\mathcal{E}$ satisfies $\psi$ with execution steps defined by $\psi^{\tau}$, then $\mathcal{E}$ satisfies $\phi$ with execution steps defined by $\mathbb{F}\left(\psi^{\tau}\right)$. The injectivity is guaranteed because $\mathbb{F}$ is component-wise injective. Definition 2 is unchanged for injective correspondences.

Example 2 Referring to the example $G_{0}$ of Section 2, the correspondence

$$
\text { inj-event }\left(e_{B}(x, y, z)\right) \Rightarrow \operatorname{inj-event}\left(e_{A}(x, y, z)\right)
$$

means that, with overwhelming probability, each execution of $e_{B}(x, y, z)$ corresponds to a distinct execution of $e_{A}(x, y, z)$. In this case, $\psi^{\tau}$ is simply the execution step of $e_{B}(x, y, z)$ and $\phi^{\tau}$ the execution step of $e_{A}(x, y, z)$. The function $\mathbb{F}$ is an injective function that maps the execution step of $e_{B}(x, y, z)$ to the execution step of $e_{A}(x, y, z)$. (This step is never $\perp$.)

The correspondence

$$
\begin{gathered}
\operatorname{event}\left(e_{1}(x)\right) \wedge \operatorname{inj-event}\left(e_{2}(x)\right) \Rightarrow \operatorname{inj-event}\left(e_{3}(x)\right) \vee \\
\left(\operatorname{inj-event}\left(e_{4}(x, y)\right) \wedge \operatorname{inj-event}\left(e_{5}(x, y)\right)\right)
\end{gathered}
$$

means that, with overwhelming probability, for all $x$, if $e_{1}(x)$ has been executed, then each execution of $e_{2}(x)$ corresponds to distinct executions of $e_{3}(x)$ or to distinct executions of $e_{4}(x, y)$ and $e_{5}(x, y)$. The function $\mathbb{F}$ maps $\perp \wedge \tau_{2}$ to $\tau_{3} \vee\left(\tau_{4} \wedge \tau_{5}\right)$, where $\tau_{2}, \tau_{3}, \tau_{4}, \tau_{5}$ are the execution steps of $e_{2}(x), e_{3}(x), e_{4}(x, y), e_{5}(x, y)$ respectively (either $\tau_{3}$ or $\tau_{4}$ and $\tau_{5}$ may be $\perp$ ). The projections of $\mathbb{F}$ map $\perp \wedge \tau_{2}$ to $\tau_{3}, \tau_{4}$, and $\tau_{5}$ respectively.

When no injective event occurs in $\psi \Rightarrow \phi$, Definition 3 reduces to the definition of non-injective correspondences.

\subsection{Property}

The next lemma is straightforward. It shows that correspondences are preserved by adding a context.

Lemma 1 If $Q$ satisfies a correspondence $c$ with public variables $V$ and $C$ is an evaluation context acceptable for $Q$ with public variables $V$ that does not contain events used by $c$, then for all $V^{\prime} \subseteq V \cup \operatorname{var}(C), C[Q]$ satisfies $c$ with public variables $V^{\prime}$.

\section{Observational Equivalence}

The notion of computational indistinguishability is key to proofs by sequences of games. In this work, we name it observational equivalence as it can be seen as an adaptation to the computational model of the notion of observational equivalence used in the spi calculus [3] in the Dolev-Yao model. We adapt the definition observational equivalence to the presence of events and review its properties.

In the next definition, we use an evaluation context $C$ to represent an algorithm that tries to distinguish $Q$ from $Q^{\prime}$.

Definition 4 (Observational equivalence) Let $Q$ and $Q^{\prime}$ be two processes that satisfy the well-formedness invariants. Let $V$ be a set of variables defined in $Q$ and $Q^{\prime}$, with the same types.

We say that $Q$ and $Q^{\prime}$ are observationally equivalent with public variables $V$, written $Q \approx^{V} Q^{\prime}$, when for all evaluation contexts $C$ acceptable for $Q$ and $Q^{\prime}$ with public variables $V$, for all distinguishers $D, \mid \operatorname{Pr}[C[Q]: D]-$ $\operatorname{Pr}\left[C\left[Q^{\prime}\right]: D\right] \mid$ is negligible.

This definition formalizes that the probability that an algorithm $C$ distinguishes the games $Q$ and $Q^{\prime}$ is negligible. The context $C$ is allowed to access directly the variables in $V$ (using find). When $V$ is empty, we write $Q \approx Q^{\prime}$.

This definition makes events observable, so that observationally equivalent processes execute computationally indistinguishable sequences of events. In a previous definition [21], in a calculus without events, the observable actions were outputs on public channels. In this definition, they are indirectly observable, since the context $C$ can receive messages output on public channels and trigger an event when a particular message is sent on a particular channel. 
The following lemma is straightforward:

Lemma $21 . \approx^{V}$ is reflexive, symmetric, and transitive.

2. If $Q \approx^{V} Q^{\prime}$ and $C$ is an evaluation context acceptable for $Q$ and $Q^{\prime}$ with public variables $V$, then for all $V^{\prime} \subseteq V \cup \operatorname{var}(C), C[Q] \approx^{V^{\prime}} C\left[Q^{\prime}\right]$.

3. If $Q \approx^{V} Q^{\prime}$ and $Q$ satisfies a correspondence $c$ with public variables $V$, then so does $Q^{\prime}$.

The transitivity of $\approx^{V}$ and Property 3 of Lemma 2 are key to performing proofs by sequences of games. Indeed, our prover starts from a game $G_{0}$ corresponding to the real protocol, and builds a sequence of observationally equivalent games $G_{0} \approx^{V} G_{1} \approx^{V} \ldots \approx^{V} G_{m}$. By transitivity, we conclude that $G_{0} \approx^{V} G_{m}$. By Property 3, if $G_{m}$ satisfies a certain correspondence with public variables $V$, then so does $G_{0}$. The sequence $G_{0} \approx^{V} G_{1} \approx^{V} \ldots \approx^{V} G_{m}$ is built by game transformations. Some of these transformations rely on security assumptions of cryptographic primitives; others are syntactic transformations used to simplify games. Since these transformations are the same for correspondences as for secrecy, we do not detail them here, and refer the reader to $[20,21]$. (These transformations leave events unchanged.) Next, we illustrate them on an example.

\section{A Proof by a Sequence of Games}

In this section, we explain the transformations performed on the process $G_{0}$ of Section 2. By the unforgeability of signatures, the signature verification with $p k_{A}$ succeeds only for signatures generated with $s k_{A}$. So, when we verify that the signature is correct, we can furthermore check that it has been generated using $s k_{A}$. So, after game transformations explained below, we obtain the following final game:

$$
\begin{aligned}
& G_{1}=c_{0}() ; \text { new } r k_{A}: \text { keyseed; } \\
& \text { let } p k_{A}=\operatorname{pkgen}^{\prime}\left(r k_{A}\right) \text { in } \overline{c_{1}}\left\langle p k_{A}\right\rangle ;\left(Q_{1 A} \mid Q_{1 B}\right) \\
& Q_{1 A}=!^{i_{A} \leq n} c_{2}\left[i_{A}\right]\left(x_{N}: \text { nonce, } x_{B} \text { : host }\right) \text {; } \\
& \text { event } e_{A}\left(p k_{A}, x_{B}, x_{N}\right) \text {; } \\
& \text { let } m=\operatorname{concat}\left(p k_{A}, x_{B}, x_{N}\right) \text { in } \\
& \text { new } r \text { : seed; } \overline{c_{3}\left[i_{A}\right]}\left\langle\operatorname{sign}^{\prime}\left(m, \operatorname{skgen}^{\prime}\left(r k_{A}\right), r\right)\right\rangle \\
& Q_{1 B}=!^{i_{B} \leq n} c_{4}\left[i_{B}\right]\left(x_{p k_{A}}: \text { pkey }\right) ; \text { new } N \text { : nonce; } \\
& \overline{c_{5}\left[i_{B}\right]}\langle N, B\rangle ; c_{6}\left[i_{B}\right](s \text { : signature }) ; \\
& \text { find } u \leq n \text { suchthat defined }\left(m[u], x_{B}[u], x_{N}[u]\right) \\
& \wedge\left(x_{p k_{A}}=p k_{A}\right) \wedge\left(B=x_{B}[u]\right) \wedge\left(N=x_{N}[u]\right) \\
& \wedge \operatorname{verify}^{\prime}\left(\operatorname{concat}\left(x_{p k_{A}}, B, N\right), x_{p k_{A}}, s\right) \text { then } \\
& \text { event } \left.e_{B}\left(x_{p k_{A}}, B, N\right)\right)
\end{aligned}
$$

The assignment $s k_{A}=\operatorname{skgen}\left(r k_{A}\right)$ has been removed and $\operatorname{skgen}\left(r k_{A}\right)$ has been substituted for $s k_{A}$, in order to make the term $\operatorname{sign}\left(m, \operatorname{skgen}\left(r k_{A}\right), r\right)$ appear. This term is needed for the security of the signature scheme to apply.

In $Q_{1 A}$, the signed message is stored in variable $m$, and this variable is used when computing the signature.

Finally, using the unforgeability of signatures, the signature verification has been replaced with an array lookup: the signature verification can succeed only when $\operatorname{concat}\left(x_{p k_{A}}, B, N\right)$ has been signed with $s k_{A}$, so we look for the message concat $\left(x_{p k_{A}}, B, N\right)$ in the array $m$ and the event $e_{B}$ is executed only when this message is found. In other words, we look for an index $u \leq n$ such that $m[u]$ is defined and $m[u]=\operatorname{concat}\left(x_{p k_{A}}, B, N\right)$. By definition of $m, m[u]=\operatorname{concat}\left(p k_{A}, x_{B}[u], x_{N}[u]\right)$, so the equality $m[u]=\operatorname{concat}\left(x_{p k_{A}}, B, N\right)$ can be replaced with $\left(x_{p k_{A}}=p k_{A}\right) \wedge\left(B=x_{B}[u]\right) \wedge\left(N=x_{N}[u]\right)$. (Recall that the result of the concat function contains enough information to recover its arguments.) This transformation replaces the function symbols pkgen, skgen, sign, and verify with primed function symbols pkgen', skgen', sign', and verify' respectively, to avoid repeated applications of the unforgeability of signatures with the same key. (The unforgeability of signatures is applied only to unprimed symbols.)

The soundness of the game transformations shows that $G_{0} \approx G_{1}$. We will prove that $G_{1}$ satisfies the correspondences (1) and (2) with any public variables $V$, in particular with $V=\emptyset$. By Lemma 2, Property 3, $G_{0}$ also satisfies these correspondences with public variables $V=\emptyset$. Let us sketch how the proof of correspondence (1) for the game $G_{1}$ will proceed. Let $Q_{1 A}^{\prime}$ and $Q_{1 B}^{\prime}$ such that $Q_{1 A}=!^{i_{A} \leq n} Q_{1 A}^{\prime}$ and $Q_{1 B}=!^{i_{B} \leq n} Q_{1 B}^{\prime}$. Assume that event $e_{B}$ is executed in the copy of $Q_{1 B}^{\prime}$ of index $i_{B}$, that is, $e_{B}\left(x_{p k_{A}}\left[i_{B}\right], B, N\left[i_{B}\right]\right)$ is executed. (Recall that the variables $x_{p k_{A}}, N, u, \ldots$ are implicitly arrays.) Then the condition of the find above $e_{B}$ holds, that is, $m\left[u\left[i_{B}\right]\right]$, $x_{B}\left[u\left[i_{B}\right]\right]$, and $x_{N}\left[u\left[i_{B}\right]\right]$ are defined, $x_{p k_{A}}\left[i_{B}\right]=p k_{A}$, $B=x_{B}\left[u\left[i_{B}\right]\right]$, and $N\left[i_{B}\right]=x_{N}\left[u\left[i_{B}\right]\right]$. Moreover, since $m\left[u\left[i_{B}\right]\right]$ is defined, the assignment that defines $m$ has been executed in the copy of $Q_{1 A}^{\prime}$ of index $i_{A}=u\left[i_{B}\right]$. Then the event $e_{A}\left(p k_{A}, x_{B}, x_{N}\right)$, located above the definition of $m$, must have been executed in that copy of $Q_{1 A}^{\prime}$, that is, $e_{A}\left(p k_{A}, x_{B}\left[u\left[i_{B}\right]\right], x_{N}\left[u\left[i_{B}\right]\right]\right)$ has been executed. The equalities in the condition of the find imply that this event is also $e_{A}\left(x_{p k_{A}}\left[i_{B}\right], B, N\left[i_{B}\right]\right)$. To sum up, if $e_{B}\left(x_{p k_{A}}\left[i_{B}\right]\right.$, $\left.B, N\left[i_{B}\right]\right)$ has been executed, then $e_{A}\left(x_{p k_{A}}\left[i_{B}\right], B, N\left[i_{B}\right]\right)$ has been executed, so we have the correspondence (1). This reasoning is typical of the way the prover shows correspondences. In particular, the conditions of array lookups are key in these proofs, because they allow us to relate values in processes that run in parallel (here, the processes that represent $A$ and $B$ ), and interesting correspondences relate events that occur in such processes. In the next section, we detail and formalize this reasoning, both for non-injective and injective correspondences. 


\section{Proving Correspondences}

In this section, we explain how our prover shows that a game satisfies a correspondence. We first sketch the technique we use for collecting properties of games, then we handle the simpler case of non-injective correspondences, before generalizing to injective correspondences.

\subsection{Reasoning on Games}

The proof of correspondences relies on two techniques for reasoning on games. These techniques were already used for simplifying games, so we summarize them briefly and refer the reader to [20] or to Appendix B for details.

First, we collect facts that hold at each program point in the game. We use the following facts: the term $M$ means that $M$ is true, defined $(M)$ means that $M$ is defined, and event $\left(e\left(M_{1}, \ldots, M_{m}\right)\right)$ means that the event $e\left(M_{1}, \ldots, M_{m}\right)$ has been executed. The set of true facts collected at program point $P$ is denoted by $\mathcal{F}_{P}$. We collect these facts as follows:

- We take into account facts that come from assignments and tests above $P$. For example, in the process if $M$ then $P$, we have $M \in \mathcal{F}_{P}$, since $M$ is true when $P$ is executed.

In our running example $G_{1}$, at the program point $P$ just after the event $e_{B}, \mathcal{F}_{P}$ contains defined $\left(m\left[u\left[i_{B}\right]\right]\right)$, defined $\left(x_{B}\left[u\left[i_{B}\right]\right]\right)$, defined $\left(x_{N}\left[u\left[i_{B}\right]\right]\right), x_{p k_{A}}\left[i_{B}\right]=$ $p k_{A}, B=x_{B}\left[u\left[i_{B}\right]\right]$, and $N\left[i_{B}\right]=x_{N}\left[u\left[i_{B}\right]\right]$, because the condition of find holds when $P$ is executed. $\left(\mathcal{F}_{P}\right.$ also contains other facts, which are useless for proving the desired correspondences, so we do not list them.)

- When we already know that $x[\widetilde{M}]$ is defined at $P$ (that is, defined $(M) \in \mathcal{F}_{P}$ and $x[\widetilde{M}]$ is a subterm of $M$ ), some definition of $x \widetilde{[i]}$ must have been executed, with $\widetilde{i}=\widetilde{M}$, so the facts $F$ that hold at all definitions of $x$ also hold at $P$, for $\widetilde{i}=\widetilde{M}: F\{\widetilde{M} \widetilde{i}\} \in \mathcal{F}_{P}$.

In the example $G_{1}$, we have $\operatorname{defined}\left(m\left[u\left[i_{B}\right]\right]\right) \in$ $\mathcal{F}_{P}$, and, when $m\left[i_{A}\right]$ is defined, event $\left(e_{A}\left(p k_{A}\right.\right.$, $\left.\left.x_{B}\left[i_{A}\right], x_{N}\left[i_{A}\right]\right)\right)$ holds, so event $\left(e_{A}\left(p k_{A}, x_{B}\left[i_{A}\right]\right.\right.$, $\left.\left.x_{N}\left[i_{A}\right]\right)\right)\left\{u\left[i_{B}\right] / i_{A}\right\} \in \mathcal{F}_{P}$, that is, event $\left(e_{A}\left(p k_{A}\right.\right.$, $\left.\left.x_{B}\left[u\left[i_{B}\right]\right], x_{N}\left[u\left[i_{B}\right]\right]\right)\right) \in \mathcal{F}_{P}$. In order words, since $m$ is defined at index $u\left[i_{B}\right]$, event $e_{A}$ has been executed in the copy of $Q_{1 A}^{\prime}$ of index $u\left[i_{B}\right]$.

Second, we use an equational prover, inspired by the Knuth-Bendix completion algorithm [41]. From a set of facts $\mathcal{F}$, it generates rewrite rules by orienting equalities of $\mathcal{F}$, and uses these rewrite rules to infer new facts from the elements of $\mathcal{F}$. It also takes into account that collisions between uniformly distributed random elements of a large type have negligible probability, so it transforms an equality $x[\widetilde{M}]=x\left[\widetilde{M^{\prime}}\right]$ into $\widetilde{M}=\widetilde{M^{\prime}}$ when $x$ is defined only by restrictions new $x: T$ and $T$ is a large type. (If the indices were different, the considered cells of $x$ would contain independent random numbers chosen uniformly in the large type $T$, so the probability of equality would be negligible.)

We say that $\mathcal{F}$ yields a contradiction when the equational prover can derive false from $\mathcal{F}$ (for example, when $\mathcal{F}$ contains an inequality $M_{1} \neq M_{2}$, rewritten by the rewrite rules into $M \neq M$, which is then rewritten into false).

\subsection{Non-injective Correspondences}

Intuitively, in order to prove that a process $Q_{0}$ satisfies a non-injective correspondence $\psi \Rightarrow \phi$, we collect all facts that hold at events in $\psi$ and show that these facts imply $\phi$ using the equational prover.

We collect facts that hold when the event $F$ in $\psi$ has been executed, as follows.

Definition 5 ( $P$ follows $\left.F, \mathcal{F}_{F, P}\right)$ When $F=\operatorname{event}\left(e\left(M_{1}\right.\right.$, $\left.\left.\ldots, M_{m}\right)\right)$ and $P$ is such that event $e\left(M_{1}^{\prime}, \ldots, M_{m}^{\prime}\right) ; P$ occurs in $Q_{0}$, we say that $P$ follows $F$, and we define $\mathcal{F}_{F, P}=\theta^{\prime} \mathcal{F}_{P} \cup\left\{\theta^{\prime} M_{j}^{\prime}=M_{j} \mid j \leq m\right\}$ where the substitution $\theta^{\prime}$ is a renaming of the replication indices at $P$ to distinct fresh replication indices.

Intuitively, when the event $F$ in $\psi$ has been executed, it has been executed by some subprocess of $Q_{0}$, so there exists a subprocess event $e\left(M_{1}^{\prime}, \ldots, M_{m}^{\prime}\right) ; P$ in $Q_{0}$ such that, for some replication indices defined by $\theta^{\prime}$, the event $e\left(M_{1}^{\prime}, \ldots, M_{m}^{\prime}\right)$ has been executed and it is equal to the event $F$, hence $\theta^{\prime} M_{j}^{\prime}=M_{j}$ holds for $j \leq m$. Moreover, since the program point $P$, which follows $F$, has been reached, $\theta^{\prime} \mathcal{F}_{P}$ holds. Hence $\mathcal{F}_{F, P}=\theta^{\prime} \mathcal{F}_{P} \cup\left\{\theta^{\prime} M_{j}^{\prime}=\right.$ $\left.M_{j} \mid j \leq m\right\}$ holds.

Let $\theta$ be a substitution equal to the identity on the variables of $\psi$. This substitution gives values to existentially quantified variables of $\phi$. We say that $\mathcal{F} \Leftrightarrow_{\theta} \phi$ when we can show that $\mathcal{F}$ implies $\theta \phi$. Formally, we define:

$\mathcal{F} \Leftrightarrow_{\theta} M$ if and only if $\mathcal{F} \cup\{\neg \theta M\}$ yields a contradiction $\mathcal{F} \Leftrightarrow_{\theta}$ event $\left(e\left(M_{1}, \ldots, M_{m}\right)\right)$ if and only if there exist $M_{1}^{\prime}, \ldots, M_{m}^{\prime}$ such that event $\left(e\left(M_{1}^{\prime}, \ldots, M_{m}^{\prime}\right)\right) \in \mathcal{F}$ and $\mathcal{F} \cup\left\{\bigvee_{j=1}^{m} \theta M_{j} \neq M_{j}^{\prime}\right\}$ yields a contradiction

$\mathcal{F} \Leftrightarrow_{\theta} \phi_{1} \wedge \phi_{2}$ if and only if $\mathcal{F} \models_{\theta} \phi_{1}$ and $\mathcal{F} \models_{\theta} \phi_{2}$ $\mathcal{F} \Leftrightarrow_{\theta} \phi_{1} \vee \phi_{2}$ if and only if $\mathcal{F} \models_{\theta} \phi_{1}$ or $\mathcal{F} \models_{\theta} \phi_{2}$

Terms $\theta M$ are proved by contradiction, using the equational prover. Events $\theta F$ are proved by looking for some event $F^{\prime}$ in $\mathcal{F}$ and showing by contradiction that $\theta F=F^{\prime}$, using the equational prover.

Non-injective correspondences are proved as follows. 
Proposition 1 Let $\psi \Rightarrow \phi$ be a non-injective correspondence, with $\psi=F_{1} \wedge \ldots \wedge F_{m}$. If for every $P_{1}$ that follows $F_{1}, \ldots$, for every $P_{m}$ that follows $F_{m}$, there exists a substitution $\theta$ equal to the identity on the variables of $\psi$ and such that $\mathcal{F}_{F_{1}, P_{1}} \cup \ldots \cup \mathcal{F}_{F_{m}, P_{m}} \Rightarrow_{\theta} \phi$, then $Q_{0}$ satisfies $\psi \Rightarrow \phi$ with any public variables $V$.

Intuitively, when $\psi=F_{1} \wedge \ldots \wedge F_{m}$ holds, $\mathcal{F}_{F_{1}, P_{1}} \cup$ $\ldots \cup \mathcal{F}_{F_{m}, P_{m}}$ hold. For some $\theta$ equal to the identity on $\psi, \mathcal{F}_{F_{1}, P_{1}} \cup \ldots \cup \mathcal{F}_{F_{m}, P_{m}}$ implies $\theta \phi$, so $\theta \phi$ holds. Hence the correspondence is satisfied. This result is proved in Appendix C.1.

Example 3 Let us prove that the example $G_{1}$ satisfies (1). For $\psi=F=\operatorname{event}\left(e_{B}(x, y, z)\right)$, the only process $P$ that follows $F$ is the process after event $e_{B}\left(x_{p k_{A}}, B, N\right)$, so this event has been executed in some copy of $Q_{1 B}^{\prime}$ of index $i_{B}^{\prime}$, with $x_{p k_{A}}\left[i_{B}^{\prime}\right]=x, B=y, N\left[i_{B}^{\prime}\right]=z$. Then, when $\psi$ holds, the facts $\mathcal{F}_{F, P}=\mathcal{F}_{P}\left\{i_{B}^{\prime} / i_{B}\right\} \cup\left\{x_{p k_{A}}\left[i_{B}^{\prime}\right]=x, B=\right.$ $\left.y, N\left[i_{B}^{\prime}\right]=z\right\}$ hold for some value of $i_{B}^{\prime}$, where $\mathcal{F}_{P}$ has been studied in Section 6.1 and $\theta^{\prime}=\left\{i_{B}^{\prime} / i_{B}\right\}$.

Furthermore, the substitution $\theta$ is the identity since all variables of $\phi$ also occur in $\psi$. Then we just have to show that $\mathcal{F}_{F, P}$ implies $\phi=\operatorname{event}\left(e_{A}(x, y, z)\right)$, that is, $\mathcal{F}_{F, P} \Rightarrow_{\theta}$ event $\left(e_{A}(x, y, z)\right)$. Since event $\left(e_{A}\left(p k_{A}\right.\right.$, $\left.\left.x_{B}\left[u\left[i_{B}\right]\right], x_{N}\left[u\left[i_{B}\right]\right]\right)\right) \in \mathcal{F}_{P}$, we have event $\left(e_{A}\left(p k_{A}\right.\right.$, $\left.\left.x_{B}\left[u\left[i_{B}^{\prime}\right]\right], x_{N}\left[u\left[i_{B}^{\prime}\right]\right]\right)\right) \in \mathcal{F}_{F, P}$, so the equational prover just has to prove by contradiction that $e_{A}\left(p k_{A}, x_{B}\left[u\left[i_{B}^{\prime}\right]\right]\right.$, $\left.x_{N}\left[u\left[i_{B}^{\prime}\right]\right]\right)=e_{A}(x, y, z)$, that is, $p k_{A}=x, x_{B}\left[u\left[i_{B}^{\prime}\right]\right]=y$, and $x_{N}\left[u\left[i_{B}^{\prime}\right]\right]=z$. The proof succeeds using the following equalities of $\mathcal{F}_{F, P}: x_{p k_{A}}\left[i_{B}^{\prime}\right]=x, B=y, N\left[i_{B}^{\prime}\right]=z$, $x_{p k_{A}}\left[i_{B}\right]=p k_{A}, B=x_{B}\left[u\left[i_{B}^{\prime}\right]\right]$, and $N\left[i_{B}^{\prime}\right]=x_{N}\left[u\left[i_{B}^{\prime}\right]\right]$.

Hence, $G_{1}$ satisfies (1) with any public variables $V$ : if $\psi=\operatorname{event}\left(e_{B}(x, y, z)\right)$ has been executed, then $\phi=$ event $\left(e_{A}(x, y, z)\right)$ has been executed.

In the implementation, the substitution $\theta$ is initially defined as the identity on $\operatorname{var}(\psi)$. It is defined on other variables when checking $\mathcal{F} \models_{\theta} M$ by trying to find $\theta$ such that $\theta M \in \mathcal{F}$, and when checking $\mathcal{F} \Leftrightarrow_{\theta} \operatorname{event}\left(e\left(M_{1}\right.\right.$, $\left.\left.\ldots, M_{m}\right)\right)$ by trying to find $\theta$ such that $\theta$ event $\left(e\left(M_{1}, \ldots\right.\right.$, $\left.\left.M_{m}\right)\right) \in \mathcal{F}$. When we do not manage to find the image by $\theta$ of all variables of $M$, resp. $M_{1}, \ldots, M_{m}$, the check fails. When there are several suitable facts $\theta M \in \mathcal{F}$ or $\theta$ event $\left(e\left(M_{1}, \ldots, M_{m}\right)\right) \in \mathcal{F}$, the system tries all possibilities.

\subsection{Injective Correspondences}

Injective correspondences are more difficult to check than non-injective ones, because they require distinguishing between several executions of the same event. We achieve that as follows.
We require that in the initial game of the sequence, which represents the real protocol, if the event $e$ is used as injective event in a correspondence, then two occurrences of $e$ always occur in different branches of find or if. This property is preserved by the game transformations, so the game $Q_{0}$ on which we test the correspondences satisfies this property. This property guarantees that for each value of the replication indices, each injective event is executed at most once.

We add as first argument of every event in $Q_{0}$ the tuple $\left(i_{1}, \ldots, i_{m}\right)$ of replication indices at the program point at which the event is executed. We add as first argument of every event in $\psi \Rightarrow \phi$ a fresh variable. Then the initial process satisfies the initial correspondence if and only if the modified process satisfies the modified correspondence. The addition of replication indices to events allows us to distinguish executions of the same injective event: these executions always have distinct replication indices by the requirement of the previous paragraph.

We extend Definition 5 to injective events, with exactly the same definition as for non-injective events. We let $I_{P}$ be the image by $\theta^{\prime}$ of the tuple of replication indices at $P$, where $\theta^{\prime}$ is the renaming defined in Definition 5.

The proof of injective correspondences extends that for non-injective correspondences: for a correspondence $\psi \Rightarrow$ $\phi$, we additionally prove that distinct executions of the injective events of $\psi$ correspond to distinct executions of each injective event of $\phi$, that is, if the injective events of $\psi$ have different replication indices, then each injective event of $\phi$ has different replication indices. In order to achieve this proof, we collect information on the replication indices of events, for each injective event of $\phi$ :

- the set of facts $\mathcal{F}$ that are known to hold, which will be used to reason on replication indices of events;

- the replication indices of the considered injective event of $\phi$, stored in a tuple $M_{0}$; these indices are computed when we prove that this event is executed;

- the replication indices of the injective events of $\psi$, stored as a mapping $\mathcal{I}=\left\{j \mapsto I_{P_{j}} \mid F_{j}\right.$ is an injective event $\}$, where $\psi=F_{1} \wedge \ldots \wedge F_{m}$ and $P_{j}$ is the process that executes $F_{j}$, for $j \leq m$;

- the set $\mathcal{V}$ containing the replication indices in $\mathcal{F}$ and the variables of $\psi$; these variables will be renamed to fresh variables in order to avoid conflicts of variable names between different events.

This information is stored in a set $\mathcal{S}$, which contains quadruples $\left(\mathcal{F}, M_{0}, \mathcal{I}, \mathcal{V}\right)$. We will show that, if the replication indices of two executions of the injective events of $\psi$ are different, then the replication indices of the corresponding executions of the considered injective event of $\phi$ are also different. Formally, we consider $\left(\mathcal{F}, M_{0}, \mathcal{I}, \mathcal{V}\right)$ and 
$\left(\mathcal{F}^{\prime}, M_{0}^{\prime}, \mathcal{I}^{\prime}, \mathcal{V}^{\prime}\right)$ in $\mathcal{S}$. We rename the variables $\mathcal{V}^{\prime}$ of the second element to fresh variables by a substitution $\theta^{\prime \prime}$ and show that, if $\mathcal{I} \neq \theta^{\prime \prime} \mathcal{I}^{\prime}$, then $M_{0} \neq \theta^{\prime \prime} M_{0}^{\prime}$ (knowing $\mathcal{F}$ and $\theta^{\prime \prime} \mathcal{F}^{\prime}$ ). This property implies injectivity.

Since this reasoning is done for each injective event in $\phi$, we collect the associated sets $\mathcal{S}$ in a pseudo-formula $\mathcal{C}$, obtained by replacing each injective event of $\phi$ with a set $\mathcal{S}$ and all other leaves of $\phi$ with $\perp$.

We say that $\vdash \mathcal{C}$ when for all non-bottom leaves $\mathcal{S}$ of $\mathcal{C}$, for all $\left(\mathcal{F}, M_{0}, \mathcal{I}, \mathcal{V}\right),\left(\mathcal{F}^{\prime}, M_{0}^{\prime}, \mathcal{I}^{\prime}, \mathcal{V}^{\prime}\right)$ in $\mathcal{S}, \mathcal{F} \cup \theta^{\prime \prime} \mathcal{F}^{\prime} \cup$ $\left\{\bigvee_{j \in \operatorname{Dom}(\mathcal{I})} \mathcal{I}(j) \neq \theta^{\prime \prime} \mathcal{I}^{\prime}(j), M_{0}=\theta^{\prime \prime} M_{0}^{\prime}\right\}$ yields a contradiction where the substitution $\theta^{\prime \prime}$ is a renaming of variables in $\mathcal{V}^{\prime}$ to distinct fresh variables. As explained above, the condition $\vdash \mathcal{C}$ guarantees injectivity.

We extend the definition of $\mathcal{F} \Rightarrow_{\theta} \phi$ used for noninjective correspondences to $\mathcal{F} \models_{\theta}^{\mathcal{I}, \mathcal{V}, \mathcal{C}} \phi$, which means that $\mathcal{F}$ implies $\theta \phi$ and $\mathcal{C}$ correctly collects the tuples $\left(\mathcal{F}, M_{0}, \mathcal{I}, \mathcal{V}\right)$ associated to this proof. Formally, we define: $\mathcal{F} \Leftrightarrow{ }_{\theta}^{\mathcal{I}, \mathcal{V}, \perp} M$ if and only if

$\mathcal{F} \cup\{\neg \theta M\}$ yields a contradiction

$\mathcal{F} \Leftrightarrow_{\theta}^{\mathcal{I}, \mathcal{V}, \perp} \operatorname{event}\left(e\left(i, M_{1}, \ldots, M_{m}\right)\right)$ if and only if

there exist $M_{0}^{\prime}, M_{1}^{\prime}, \ldots, M_{m}^{\prime}$ such that

event $\left(e\left(M_{0}^{\prime}, M_{1}^{\prime}, \ldots, M_{m}^{\prime}\right)\right) \in \mathcal{F}$ and $\mathcal{F} \cup$

$\left\{\theta i \neq M_{0}^{\prime} \vee \bigvee_{j=1}^{m} \theta M_{j} \neq M_{j}^{\prime}\right\}$ yields a contradiction

$\mathcal{F} \models_{\theta}^{\mathcal{I}, \mathcal{V}, \mathcal{S}}$ inj-event $\left(e\left(i, M_{1}, \ldots, M_{m}\right)\right)$ if and only if

there exist $M_{0}^{\prime}, M_{1}^{\prime}, \ldots, M_{m}^{\prime}$ such that

event $\left(e\left(M_{0}^{\prime}, M_{1}^{\prime}, \ldots, M_{m}^{\prime}\right)\right) \in \mathcal{F}$,

$\mathcal{F} \cup\left\{\theta i \neq M_{0}^{\prime} \vee \bigvee_{j=1}^{m} \theta M_{j} \neq M_{j}^{\prime}\right\}$ yields a

contradiction, and $\left(\mathcal{F}, M_{0}^{\prime}, \mathcal{I}, \mathcal{V}\right) \in \mathcal{S}$.

$\mathcal{F} \Leftrightarrow_{\theta}^{\mathcal{I}, \mathcal{V}, \mathcal{C}_{1} \wedge \mathcal{C}_{2}} \phi_{1} \wedge \phi_{2}$ if and only if

$\mathcal{F} \Leftrightarrow_{\theta}^{\mathcal{I}, \mathcal{V}, \mathcal{C}_{1}} \phi_{1}$ and $\mathcal{F} \Leftrightarrow_{\theta}^{\mathcal{I}, \mathcal{V}, \mathcal{C}_{2}} \phi_{2}$

$\mathcal{F} \Leftrightarrow_{\theta}^{\mathcal{I}, \mathcal{V}, \mathcal{C}_{1} \vee \mathcal{C}_{2}} \phi_{1} \vee \phi_{2}$ if and only if

$\mathcal{F} \Leftrightarrow_{\theta}^{\mathcal{I}, \mathcal{V}, \mathcal{C}_{1}} \phi_{1}$ or $\mathcal{F} \Leftrightarrow_{\theta}^{\mathcal{I}, \mathcal{V}, \mathcal{C}_{2}} \phi_{2}$

These formulae differ from the non-injective case in that we propagate $\mathcal{I}, \mathcal{V}, \mathcal{C}$ and, in the case of injective events, we make sure that quadruples $\left(\mathcal{F}, M_{0}^{\prime}, \mathcal{I}, \mathcal{V}\right)$ are collected correctly by requiring that $\left(\mathcal{F}, M_{0}^{\prime}, \mathcal{I}, \mathcal{V}\right) \in \mathcal{S}$.

Injective correspondences are proved as follows.

Proposition 2 Let $\psi \Rightarrow \phi$ be a correspondence, with $\psi=$ $F_{1} \wedge \ldots \wedge F_{m}$.

Assume that, for all events e used as injective events in $\psi \Rightarrow \phi$, two occurrences of the event e always occur in different branches of find or if in $Q_{0}$.

Assume that there exists $\mathcal{C}$ such that $\vdash \mathcal{C}$ and for every $P_{1}$ that follows $F_{1}, \ldots$, for every $P_{m}$ that follows $F_{m}$, there exists a substitution $\theta$ equal to the identity on the variables of $\psi$ and such that $\mathcal{F}_{F_{1}, P_{1}} \cup \ldots \cup \mathcal{F}_{F_{m}, P_{m}} \mapsto_{\theta}^{\mathcal{I}, \mathcal{V}, \mathcal{C}} \phi$ where $\mathcal{I}=\left\{j \mapsto I_{P_{j}} \mid F_{j}\right.$ is an injective event $\}$ and $\mathcal{V}=\operatorname{var}\left(I_{P_{1}}\right) \cup \ldots \cup \operatorname{var}\left(I_{P_{m}}\right) \cup \operatorname{var}(\psi)$.

Then $Q_{0}$ satisfies $\psi \Rightarrow \phi$ with any public variables $V$.
This result is proved in Appendix C.2. In the implementation, the value of $\mathcal{C}$ is computed by adding $\left(\mathcal{F}, M_{0}^{\prime}, \mathcal{I}, \mathcal{V}\right)$ to $\mathcal{S}$ when handling injective events during the checking of $\mathcal{F}_{F_{1}, P_{1}} \cup \ldots \cup \mathcal{F}_{F_{m}, P_{m}} \Rightarrow_{\theta}^{\mathcal{I}, \mathcal{V}, \mathcal{C}} \phi$.

Example 4 Let us prove that the example $G_{1}$ satisfies (2). After adding replication indices to events, the process contains events $e_{A}\left(i_{A}, p k_{A}, x_{B}, x_{N}\right)$ and $e_{B}\left(i_{B}, x_{p k_{A}}, B, N\right)$, and we prove the correspondence $\psi \Rightarrow \phi=\operatorname{inj-event}\left(e_{B}(i\right.$, $x, y, z)) \Rightarrow \operatorname{inj-event}\left(e_{A}\left(i^{\prime}, x, y, z\right)\right)$. As in Section 6.1, we compute the set $\mathcal{F}_{P}$ of facts that hold at the program point $P$ just after event $e_{B}$. However, $m$ is defined at in$\operatorname{dex} i_{A}=u\left[i_{B}\right]$ now implies that event $\left(e_{A}\left(u\left[i_{B}\right], p k_{A}\right.\right.$, $\left.\left.x_{B}\left[u\left[i_{B}\right]\right], x_{N}\left[u\left[i_{B}\right]\right]\right)\right) \in \mathcal{F}_{P}$. The process $P$ follows $F=\operatorname{event}\left(e_{B}(i, x, y, z)\right)$ and $\mathcal{F}=\mathcal{F}_{F, P}=\mathcal{F}_{P}\left\{i_{B}^{\prime} / i_{B}\right\} \cup$ $\left\{i_{B}^{\prime}=i, x_{p k_{A}}\left[i_{B}^{\prime}\right]=x, B=y, N\left[i_{B}^{\prime}\right]=z\right\}$.

Similarly to the proof of $\mathcal{F} \Leftrightarrow_{\theta}$ event $\left(e_{A}(x, y, z)\right)$ in Example 3, we can show that $\mathcal{F} \Leftrightarrow_{\theta}^{\mathcal{I}, \mathcal{V}, \mathcal{C}}$ event $\left(e_{A}\left(i^{\prime}, x, y, z\right)\right)$ where $\mathcal{I}=\left\{1 \mapsto i_{B}^{\prime}\right\}$ encodes the replication indices of the events of $\psi, \mathcal{V}=\left\{i_{B}^{\prime}, i, x, y, z\right\}$ contains the replication indices of $\mathcal{F}$ and the variables of $\psi, \mathcal{C}=$ $\mathcal{S}=\left\{\left(\mathcal{F}, u\left[i_{B}^{\prime}\right], \mathcal{I}, \mathcal{V}\right)\right\} . \quad(\mathcal{C}=\mathcal{S}$ because the formula $\psi$ is reduced to a single event; $M_{0}^{\prime}=u\left[i_{B}\right]$ contains the replication indices of the event $e_{A}$ contained in $\mathcal{F}$ : $\operatorname{event}\left(e_{A}\left(u\left[i_{B}^{\prime}\right], p k_{A}, x_{B}\left[u\left[i_{B}^{\prime}\right]\right], x_{N}\left[u\left[i_{B}^{\prime}\right]\right]\right)\right) \in \mathcal{F}$.)

In order to prove injectivity, it remains to show that $\vdash$ $\mathcal{C}$. Let $\theta^{\prime \prime}=\left\{i_{B}^{\prime \prime} / i_{B}^{\prime}, i^{\prime \prime} / i, x^{\prime \prime} / x, y^{\prime \prime} / y, z^{\prime \prime} / z\right\}$. We need to show that $\mathcal{F} \cup \theta^{\prime \prime} \mathcal{F} \cup\left\{i_{B}^{\prime} \neq i_{B}^{\prime \prime}, u\left[i_{B}^{\prime}\right]=u\left[i_{B}^{\prime \prime}\right]\right\}$ yields a contradiction, that is, if the replication indices of the event $e_{B}$ in $\psi$ are distinct $\left(i_{B}^{\prime} \neq i_{B}^{\prime \prime}\right)$, then the replication indices of the event $e_{A}$ in $\phi$ are also distinct $\left(u\left[i_{B}^{\prime}\right] \neq u\left[i_{B}^{\prime \prime}\right]\right)$.

$\mathcal{F}$ contains $N\left[i_{B}^{\prime}\right]=x_{N}\left[u\left[i_{B}^{\prime}\right]\right]$, so $\theta^{\prime \prime} \mathcal{F}$ contains $N\left[i_{B}^{\prime \prime}\right]=x_{N}\left[u\left[i_{B}^{\prime \prime}\right]\right]$. These two equalities combined with $u\left[i_{B}^{\prime}\right]=u\left[i_{B}^{\prime \prime}\right]$ imply that $N\left[i_{B}^{\prime}\right]=x_{N}\left[u\left[i_{B}^{\prime}\right]\right]=$ $x_{N}\left[u\left[i_{B}^{\prime \prime}\right]\right]=N\left[i_{B}^{\prime \prime}\right]$. Since $N$ is defined by restrictions of the large type nonce, $N\left[i_{B}^{\prime}\right]=N\left[i_{B}^{\prime \prime}\right]$ implies $i_{B}^{\prime}=i_{B}^{\prime \prime}$ with overwhelming probability, by eliminating collisions. This equality contradicts $i_{B}^{\prime} \neq i_{B}^{\prime \prime}$, so we obtain the desired injectivity and $G_{1}$ satisfies (2) with any public variables $V$.

\section{Authentication and Key Exchange}

In this section, we show how correspondences can be used to prove mutual authentication and authenticated key exchange, as formalized in cryptography following the seminal paper by Bellare and Rogaway [16] and more recent formalizations [7, 27]. Additional discussion and comparisons between these models can be found in Appendix D.

\subsection{Mutual Authentication}

For simplicity, we consider a protocol that includes two roles, initiator and responder, played by two participants $A$ 
and $B$, respectively. Other participants are included in the adversary. The protocol consists of a sequence of messages exchanged alternatively from the initiator to the responder and from the responder to the initiator. Such a configuration can be represented by a process of the form

$$
Q_{0}=\text { Init } ;\left(!^{i_{A} \leq n} Q_{A}\left|!^{i_{B} \leq n} Q_{B}\right| Q_{S}\right)
$$

where Init is an initialization process (creating keys of $A$ and $B$ for instance), $Q_{A}$ and $Q_{B}$ represent respectively the initiator $A$ and the responder $B$, and $Q_{S}$ represents a process that allows the adversary to register keys of other (possibly dishonest) participants, so that they can take part in sessions of the protocol with $A$ and $B$. The processes $Q_{A}$ and $Q_{B}$ do not contain replications.

We assume that the protocol contains an odd number of rounds $r$, so that the first and last messages of the protocol are both from the initiator to the responder. (The other case can be handled similarly.) We assume that the process $Q_{A}$ stores the messages of the protocol in variables $x_{1}, \ldots, x_{r}$ and that $Q_{B}$ stores them in variables $y_{1}, \ldots, y_{r}$. The initiator process $Q_{A}$ starts by receiving a message that is not really part of the protocol, and which contains the identity $Y$ of the responder with which $A$ is supposed to run a session. The last ( $r$-th) message sent by process $Q_{A}$ is assumed to be a pair containing, in addition to the last message of the protocol $x_{r}$, either accept ${ }_{A}(Y)$, when the protocol ran as expected, or reject, when the protocol failed. The process $Q_{B}$ is assumed to send a $(r+1)$-th message containing either accept ${ }_{B}(X)$ or reject just after $B$ received and checked the last message of the protocol, where $X$ is the identity of its expected partner (inferred by $B$ from the protocol messages). We designate by $Q_{A}^{i}$ the copy of $Q_{A}$ of index $i_{A}=i$ and by $Q_{B}^{i}$ the copy of $Q_{B}$ of index $i_{B}=i$. We say that $Q_{A}^{i}$ accepts with $B$ when it sends $\operatorname{accept}_{A}(B)$ as second component of its last message; $Q_{B}^{i}$ accepts with $A$ when it sends $\operatorname{accept}_{B}(A)$ as $(r+1)$-th message.

A session identifier is a function sid of the protocol messages; $\operatorname{sid}\left(x_{1}, \ldots, x_{r}\right)$ is typically a subsequence of the messages $x_{1}, \ldots, x_{r}$, often the whole sequence. We also define a partial session identifier $\operatorname{sid}^{\prime}\left(x_{1}, \ldots, x_{r-1}\right)$, useful since the $r$-th message is not available to $B$ when $A$ accepts. We require that $\operatorname{sid}\left(x_{1}, \ldots, x_{r}\right)=\operatorname{sid}\left(y_{1}, \ldots, y_{r}\right)$ implies $\operatorname{sid}^{\prime}\left(x_{1}, \ldots, x_{r-1}\right)=\operatorname{sid}^{\prime}\left(y_{1}, \ldots, y_{r-1}\right)$. We say that $Q_{A}^{i}$ and $Q_{B}^{i^{\prime}}$ are (real) partners when they have the same session identifier: $\operatorname{sid}\left(x_{1}[i], \ldots, x_{r}[i]\right)=\operatorname{sid}\left(y_{1}\left[i^{\prime}\right], \ldots, y_{r}\left[i^{\prime}\right]\right)$.

Definition 6 We say that $Q_{0}$ is a secure mutual authentication protocol with session identifiers sid and sid ${ }^{\prime}$ if:

1. if the adversary just sends $B$ to $Q_{A}^{i}$ as first message and relays messages faithfully between $Q_{A}^{i}$ and $Q_{B}^{i^{\prime}}$, then $Q_{A}^{i}$ accepts with $B$ and $Q_{B}^{i^{\prime}}$ accepts with $A$;
2. with overwhelming probability, there exists an injective function that maps each index $i$ of a process $Q_{A}^{i}$ that accepts with $B$ to the index $i^{\prime}$ of a process $Q_{B}^{i^{\prime}}$ with expected partner $A$ such that $\operatorname{sid}^{\prime}\left(x_{1}[i], \ldots, x_{r-1}[i]\right)=\operatorname{sid}^{\prime}\left(y_{1}\left[i^{\prime}\right], \ldots, y_{r-1}\left[i^{\prime}\right]\right)$;

3. with overwhelming probability, there exists an injective function that maps each index $i^{\prime}$ of a process $Q_{B}^{i^{\prime}}$ that accepts with $A$ to the index $i$ of a process $Q_{A}^{i}$ that accepts with $B$ such that $\operatorname{sid}\left(x_{1}[i], \ldots, x_{r}[i]\right)=$ $\operatorname{sid}\left(y_{1}\left[i^{\prime}\right], \ldots, y_{r}\left[i^{\prime}\right]\right)$.

In item $2, Q_{B}^{i^{\prime}}$ has not accepted yet when $Q_{A}^{i}$ accepts, so we cannot require that $Q_{B}^{i^{\prime}}$ accepts with $A$; we only require that $Q_{B}^{i^{\prime}}$ has expected partner $A$ (so that, if it accepts later, it accepts with $A$ ). The first condition is easy to check manually, as already noticed in [16]: it expresses that the protocol works when $A$ and $B$ interact without adversary. The last two conditions mean that each session of $A$ corresponds to a distinct session of $B$, and conversely, with overwhelming probability. They can be verified using correspondences, as shown by the following proposition.

\section{Proposition 3 Let $Q_{0}^{\prime}$ be obtained from $Q_{0}$ by adding}

- event $\operatorname{part}_{A}\left(Y, \operatorname{sid}^{\prime}\left(x_{1}, \ldots, x_{r-1}\right)\right)$; event $f_{u l l}(Y$, $\left.\operatorname{sid}\left(x_{1}, \ldots, x_{r}\right)\right)$ just before $A$ sends $x_{r}, \operatorname{accept}_{A}(Y)$;

- event $\operatorname{full}_{B}\left(X, \operatorname{sid}\left(y_{1}, \ldots, y_{r}\right)\right)$ just before $B$ sends $\operatorname{accept}_{B}(X)$;

- event $\operatorname{part}_{B}\left(X, \operatorname{sid}^{\prime}\left(y_{1}, \ldots, y_{r-1}\right)\right)$ just before $B$ sends $y_{r-1}$.

If $Q_{0}$ satisfies the first condition of Definition 6 and $Q_{0}^{\prime}$ satisfies the correspondences

$$
\begin{aligned}
& \operatorname{inj-event}\left(\operatorname{part}_{A}(B, x)\right) \Rightarrow \operatorname{inj-event}\left(\operatorname{part}_{B}(A, x)\right) \\
& \text { inj-event }\left(\operatorname{full}_{B}(A, x)\right) \Rightarrow \operatorname{inj-event}\left(\operatorname{full}_{A}(B, x)\right)
\end{aligned}
$$

with public variables $V=\emptyset$, then $Q_{0}$ is a secure mutual authentication protocol with session identifiers sid and $\mathrm{sid}^{\prime}$.

The proof of this proposition is straightforward from the definitions. Obviously, many other versions of authentication can be verified using correspondences, for example by requiring non-injective properties instead of injective ones or by requiring authentication in one direction only instead of mutual authentication.

\subsection{Authenticated Key Exchange}

We adopt the same hypotheses as for mutual authentication. Furthermore, we assume that $Q_{A}$ sends or receives the $j$-th message of the protocol on channel $c_{A j}\left[i_{A}\right]$, and similarly $Q_{B}$ on channel $c_{B j}\left[i_{B}\right]$. The channels $c_{A j}\left[i_{A}\right]$ 
and $c_{B j}\left[i_{B}\right]$ are not used for other purposes. We assume that, just before $Q_{A}$ ends accepting, it stores the established key in variable $k_{A}$ of type $T$, and sends $x_{r}$, accept ${ }_{A}(Y)$ on channel $c_{A r}\left[i_{A}\right]$. We assume that, just before $Q_{B}$ ends accepting, it stores the established key in variable $k_{B}$ of type $T$ and sends accept ${ }_{B}(X)$ on channel $c_{B r+1}\left[i_{B}\right]$.

We consider here the Real-Or-Random model [7]: the adversary is allowed to ask several test queries, which either all return the session key (real) or all return a random key (random). Our goal is to show that the adversary has a negligible probability of distinguishing these two situations. As shown in [7], the Real-Or-Random model is stronger than the Find-Then-Guess model of [16]. When the test queries return the real session key, they are defined by the process $Q_{T}=Q_{T A} \mid Q_{T B}$, where

$$
\begin{aligned}
& Q_{T A}=!^{i \leq n_{T}} \text { test }_{A}[i]\left(u_{A}\right) \\
& \quad \text { if } \operatorname{defined}\left(k_{A}\left[u_{A}\right]\right) \text { then } \overline{\text { test }_{A}[i]}\left\langle k_{A}\left[u_{A}\right]\right\rangle
\end{aligned}
$$

and $Q_{T B}$ is defined symmetrically. When the test queries return a random key, they are defined by the process $Q_{T}^{\prime}=$ $Q_{T A}^{\prime} \mid Q_{T B}^{\prime}$, where

$$
\begin{aligned}
& Q_{T A}^{\prime}=!^{i \leq n_{T}} \text { test }_{A}[i]\left(u_{A}\right) \\
& \text { if defined }\left(k_{A}\left[u_{A}\right], Y\left[u_{A}\right]\right) \text { then } \\
& \text { if } Y\left[u_{A}\right] \neq B \text { then } \overline{\text { test }_{A}[i]}\left\langle k_{A}\left[u_{A}\right]\right\rangle \text { else } \\
& \text { find } u \leq n_{T} \text { suchthat } \operatorname{defined}\left(u_{A}[u], r_{A}[u]\right) \wedge \\
& \quad u_{A}[u]=u_{A} \text { then } \overline{\operatorname{test}_{A}[i]}\left\langle r_{A}[u]\right\rangle \text { else } \\
& \text { find } u \leq n_{T} \text { suchthat } \operatorname{defined}\left(u_{B}[u], r_{B}[u],\right. \\
& \left.\quad x_{1}\left[u_{A}\right], \ldots, x_{r}\left[u_{A}\right], y_{1}\left[u_{B}[u]\right], \ldots, y_{r}\left[u_{B}[u]\right]\right) \wedge \\
& \quad \operatorname{sid}\left(x_{1}\left[u_{A}\right], \ldots, x_{r}\left[u_{A}\right]\right)=\operatorname{sid}\left(y_{1}\left[u_{B}[u]\right], \ldots,\right. \\
& \left.\quad y_{r}\left[u_{B}[u]\right]\right) \text { then } \overline{\operatorname{test}_{A}[i]}\left\langle r_{B}[u]\right\rangle \text { else } \\
& \text { new } r_{A}: T ; \overline{\text { test }_{A}[i]}\left\langle r_{A}\right\rangle
\end{aligned}
$$

and $Q_{T B}^{\prime}$ is defined symmetrically. When the expected partner of $A$ is not $B$, the session is executed with a dishonest participant; then, the test query $Q_{T A}^{\prime}$ returns the real key. When the test query $Q_{T A}^{\prime}$ has already been asked to the same copy of $Q_{A}$ (of index $u_{A}[u]=u_{A}$ ), or to a copy of $Q_{B}$ with the same session identifier (of index $u_{B}[u]$ such that $\operatorname{sid}\left(x_{1}\left[u_{A}\right], \ldots, x_{r}\left[u_{A}\right]\right)=\operatorname{sid}\left(y_{1}\left[u_{B}[u]\right]\right.$, $\left.\left.\ldots, y_{r}\left[u_{B}[u]\right]\right)\right), Q_{T A}^{\prime}$ returns the same result as in the previous test query. Otherwise, $Q_{T A}^{\prime}$ returns a fresh random key $r_{A}$.

Definition 7 We say that $Q_{0}$ is a secure authenticated key exchange over $T$ with session identifiers sid and $\operatorname{sid}^{\prime}$ if $Q_{0}$ is a secure mutual authentication protocol with session identifiers sid and $\operatorname{sid}^{\prime}$ and the following are true:

1. if the adversary just sends $B$ to $Q_{A}^{i}$ as first message and relays messages faithfully between $Q_{A}^{i}$ and
$Q_{B}^{i^{\prime}}$, then $Q_{A}^{i}$ accepts with $B, Q_{B}^{i^{\prime}}$ accepts with $A$, $k_{A}[i]=k_{B}\left[i^{\prime}\right]$, and this random variable is uniformly distributed in $T$;

2. $Q_{0}\left|Q_{T} \approx Q_{0}\right| Q_{T}^{\prime}$.

The first point of this definition means that the protocol works correctly when $A$ and $B$ interact without adversary. The second point expresses the indistinguishability between the real key (returned by $Q_{T}$ ) and a random key (returned by $\left.Q_{T}^{\prime}\right)$.

As shown in [20,21], our prover can prove the secrecy of a variable $x$, defined as follows:

Definition 8 (Secrecy) Assume $x$ of type $T$ is defined in $Q$ under a single replication $!^{i \leq n}$. Let $Q^{\prime}$ be obtained from $Q$ by removing events. The process $Q$ preserves the secrecy of $x$ when $Q^{\prime}\left|R_{x} \approx Q^{\prime}\right| R_{x}^{\prime}$, where

$$
\begin{aligned}
& R_{x}=!^{i \leq n^{\prime}} c[i](u:[1, n]) ; \text { if } \operatorname{defined}(x[u]) \text { then } \overline{c[i]}\langle x[u]\rangle \\
& R_{x}^{\prime}=!^{i \leq n^{\prime}} c[i](u:[1, n]) ; \text { if } \operatorname{defined}(x[u]) \text { then } \\
& \quad \text { find } u^{\prime} \leq n^{\prime} \text { suchthat defined }\left(y\left[u^{\prime}\right], u\left[u^{\prime}\right]\right) \wedge u\left[u^{\prime}\right]=u \\
& \quad \text { then } \overline{c[i]}\left\langle y\left[u^{\prime}\right]\right\rangle \text { else new } y: T ; \overline{c[i]}\langle y\rangle \\
& c \notin \operatorname{fc}\left(Q^{\prime}\right), \text { and } u, u^{\prime}, y \notin \operatorname{var}\left(Q^{\prime}\right) .
\end{aligned}
$$

Intuitively, this definition means that the adversary cannot distinguish the array $x$ from an array of uniformly distributed random values by performing several test queries represented by $R_{x}$ and $R_{x}^{\prime}$, with non-negligible probability.

Proposition 4 Let $Q_{0}^{\prime}$ be obtained from $Q_{0}$ by replacing $\overline{c_{A r}\left[i_{A}\right]}\left\langle x_{r}, \operatorname{accept}_{A}(Y)\right\rangle$ with

$$
\begin{aligned}
& \text { event } \operatorname{part}_{A}\left(Y, \operatorname{sid}^{\prime}\left(x_{1}, \ldots, x_{r-1}\right)\right) ; \\
& \text { event } f_{u l l}\left(Y, k_{A}, \operatorname{sid}\left(x_{1}, \ldots, x_{r}\right)\right) ; \\
& \text { if } Y=B \text { then } \\
& \quad \text { let } k_{A}^{\prime}=k_{A} \text { in } \overline{c_{A r}\left[i_{A}\right]}\left\langle x_{r}, \operatorname{accept}_{A}(Y)\right\rangle \\
& \text { else } \\
& \qquad \overline{c_{A r}\left[i_{A}\right]}\left\langle x_{r}, \operatorname{accept}_{A}(Y)\right\rangle ; c_{A K}\left[i_{A}\right]() ; \overline{c_{A K}\left[i_{A}\right]}\left\langle k_{A}\right\rangle \\
& \text { and } \overline{c_{B r+1}\left[i_{B}\right]}\left\langle\operatorname{accept}_{B}(X)\right\rangle \text { with } \\
& \text { event full}{ }_{B}\left(X, k_{B}, \operatorname{sid}\left(y_{1}, \ldots, y_{r}\right)\right) ; \\
& \text { if } \overline{X=A \text { then }} \overline{c_{B r+1}\left[i_{B}\right]}\left\langle\operatorname{accept}_{B}(X)\right\rangle \\
& \text { else } \overline{c_{B r+1}\left[i_{B}\right]}\left\langle\operatorname{accept}_{B}(X)\right\rangle ; c_{B K}\left[i_{B}\right]() ; \overline{c_{B K}\left[i_{B}\right]}\left\langle k_{B}\right\rangle
\end{aligned}
$$

and adding event $\operatorname{part}_{B}\left(X, \operatorname{sid}^{\prime}\left(y_{1}, \ldots, y_{r-1}\right)\right)$ just before $Q_{B}$ sends $y_{r-1}$. 
If $Q_{0}$ satisfies the first condition of Definition 7, $Q_{0}^{\prime}$ preserves the secrecy of $k_{A}^{\prime}$, and $Q_{0}^{\prime}$ satisfies the correspondences

$$
\begin{aligned}
& \operatorname{inj-event}\left(\operatorname{part}_{A}(B, x)\right) \Rightarrow \operatorname{inj-event}\left(\operatorname{part}_{B}(A, x)\right) \\
& \operatorname{inj-event}\left(f_{l} l_{B}(A, k, x)\right) \Rightarrow \operatorname{inj-event}\left(f_{l}\left(f_{A}(B, k, x)\right)\right. \\
& \text { event }\left(f_{u l l}(A)\right. \\
& B, k, x)) \wedge \operatorname{event}\left(f u l l_{A}\left(B, k^{\prime}, x\right)\right) \Rightarrow k=k^{\prime}
\end{aligned}
$$

with public variables $\left\{k_{A}^{\prime}\right\}$, then $Q_{0}$ is a secure authenticated key exchange with session identifiers sid and sid'.

This result is proved in Appendix C.3. The process $Q_{0}^{\prime}$ adds events as for mutual authentication, except that the exchanged key is added to the events full $_{A}$ and full $_{B}$. Furthermore, when $A$ runs a session with $B$, it stores the key in the variable $k_{A}^{\prime}$. When $A$ runs a session with $Y \neq B$, it allows the adversary to obtain the exchanged key, by sending a message on $c_{A K}$, and symmetrically when $B$ runs a session with $X \neq A$. (The test queries also allow the adversary to get the key in this case.) As for Proposition 3, the first condition of Definition 7 is easy to check manually. The first two correspondences imply mutual authentication. The equivalence $Q_{0}\left|Q_{T} \approx Q_{0}\right| Q_{T}^{\prime}$ is obtained by combining the last two correspondences with the secrecy of $k_{A}^{\prime}$. Intuitively, the correspondences allow us to show that each element of $k_{B}$ in a session with $A$ is in fact also an element of $k_{A}^{\prime}$ (which we can find by looking for the same session identifier), so showing that $k_{A}^{\prime}$ cannot be distinguished from an array of independent random numbers is sufficient to show the secrecy of the key. The correspondences must be true with public variables $\left\{k_{A}^{\prime}\right\}$, so that the context is allowed to access $k_{A}^{\prime}$ : in the proof, the process $Q_{0}^{\prime}$ is put in a context that implements the test queries by calling the processes $R_{k_{A}^{\prime}}$ or $R_{k_{A}^{\prime}}^{\prime}$ of Definition 8, which directly access $k_{A}^{\prime}$.

\section{Experimental Results}

We have successfully tested our prover on examples of protocols of the literature: Yahalom [23] with and without key confirmation, Otway-Rees [55], and the original and corrected versions of Woo-Lam shared-key [36] and public-key [62,64], Needham-Schroeder public-key [48, 53], Denning-Sacco public-key [5,34], and NeedhamSchroeder shared-key $[53,54]$ with and without key confirmation. For each protocol, we have tried to prove one-way or mutual authentication or authenticated key exchange, depending on the goal of the protocol. Our prover obviously does not prove properties that do not hold. It succeeds in proving properties that hold, in all cases except one: it cannot show (4) for the original version of the NeedhamSchroeder shared-key protocol, because it fails to prove that
$N_{B}[i] \neq N_{B}\left[i^{\prime}\right]-1$ with overwhelming probability, where $N_{B}$ is a nonce. ${ }^{1}$

Our prover can make subtle distinctions, which are typically not made by Dolev-Yao provers. For instance, it can model two notions of security for signatures: one in which the adversary is allowed to forge a new signature for an already signed message; the other in which the adversary cannot forge any signature. With the latter definition, for the corrected Woo-Lam public key protocol [64], it can show that the signature is authenticated (both participants have exactly the same signature), while it cannot with the former definition, because the two participants may have different signatures for the same message.

The total runtime for all these tests is $29 \mathrm{~s}$ on a Pentium M 1.8 GHz. Appendix E details these results.

\section{Conclusion}

We have presented the first tool for proving correspondences by sequences of games, in the computational model. This tool works with no or very little help from the user, handles a wide variety of cryptographic primitives, and produces proofs valid for a polynomial number of sessions in the presence of an active adversary.

Although this tool can prove complex correspondences, with conjunctions and disjunctions, our examples use rather simple ones. Complex correspondences proved useful in case studies $[1,2]$ in the Dolev-Yao model; we plan to use them in similar situations in the computational model. Our tool can also be used to analyze protocols or combinations of primitives that are outside the scope of the Dolev-Yao model. For example, in [22], in collaboration with David Pointcheval, we have used it to prove the Full Domain Hash signature scheme. We plan to consider other such examples in the future.

Acknowledgments We thank David Pointcheval for very helpful discussions on this paper. This work was partly done while the author was at CNRS, École Normale Supérieure, Paris. It was partly supported by the ANR project ARA SSIA Formacrypt.

\section{References}

[1] M. Abadi and B. Blanchet. Computer-assisted verification of a protocol for certified email. Science of Computer Programming, 58(1-2):3-27, Oct. 2005. Special issue SAS'03.

[2] M. Abadi, B. Blanchet, and C. Fournet. Just fast keying in the pi calculus. ACM Transactions on Information and System Security, 10(3):1-59, July 2007. An extended abstract appears in ESOP'04.

\footnotetext{
${ }^{1}$ This issue has been solved in CryptoVerif 1.20 , so that CryptoVerif now succeeds in proving (4) for this protocol.
} 
[3] M. Abadi and A. D. Gordon. A calculus for cryptographic protocols: The spi calculus. Information and Computation, 148(1):1-70, Jan. 1999. An extended version appeared as Digital Equipment Corporation Systems Research Center report No. 149, January 1998.

[4] M. Abadi and J. Jürjens. Formal eavesdropping and its computational interpretation. In TACS' 01 , volume 2215 of LNCS, pages 82-94. Springer, Oct. 2001.

[5] M. Abadi and R. Needham. Prudent engineering practice for cryptographic protocols. IEEE Transactions on Software Engineering, 22(1):6-15, Jan. 1996.

[6] M. Abadi and P. Rogaway. Reconciling two views of cryptography (the computational soundness of formal encryption). Journal of Cryptology, 15(2):103-127, 2002.

[7] M. Abdalla, P.-A. Fouque, and D. Pointcheval. Passwordbased authenticated key exchange in the three-party setting. IEE Proceedings Information Security, 153(1):27-39, Mar. 2006.

[8] P. Adão, G. Bana, J. Herzog, and A. Scedrov. Soundness of formal encryption in the presence of key-cycles. In $E S$ ORICS'05, volume 3679 of $L N C S$, pages 374-396. Springer, Sept. 2005.

[9] M. Backes and P. Laud. Computationally sound secrecy proofs by mechanized flow analysis. In $C C S^{\prime} 06$, pages 370 379. ACM, Nov. 2006.

[10] M. Backes and B. Pfitzmann. Symmetric encryption in a simulatable Dolev-Yao style cryptographic library. In CSFW'04, pages 204-218. IEEE, June 2004.

[11] M. Backes, B. Pfitzmann, and M. Waidner. A composable cryptographic library with nested operations. In $C_{C S}{ }^{\prime} 03$, pages 220-230. ACM, Oct. 2003.

[12] M. Backes, B. Pfitzmann, and M. Waidner. Symmetric authentication within a simulatable cryptographic library. In ESORICS'03, volume 2808 of LNCS, pages 271-290. Springer, Oct. 2003.

[13] G. Barthe, J. Cederquist, and S. Tarento. A machine-checked formalization of the generic model and the random oracle model. In IJCAR'04, volume 3097 of LNCS, pages 385399. Springer, July 2004.

[14] M. Baudet, V. Cortier, and S. Kremer. Computationally sound implementations of equational theories against passive adversaries. In ICALP'05, volume 3580 of $L N C S$, pages 652-663. Springer, July 2005.

[15] M. Bellare, D. Pointcheval, and P. Rogaway. Authenticated key exchange secure against dictionary attacks. In EUROCRYPT'OO, volume 1807 of LNCS, pages 139-155. Springer, 2000.

[16] M. Bellare and P. Rogaway. Entity authentication and key distribution. In CRYPTO'93, volume 773 of LNCS, pages 232-249. Springer, Aug. 1993.

[17] M. Bellare and P. Rogaway. The security of triple encryption and a framework for code-based game-playing proofs. In EUROCRYPT'06, volume 4004 of LNCS, pages 409-426. Springer, May 2006. Extended version available at http: //eprint.iacr.org/2004/331.

[18] B. Blanchet. From secrecy to authenticity in security protocols. In SAS'02, volume 2477 of $L N C S$, pages 342-359. Springer, Sept. 2002.
[19] B. Blanchet. Automatic proof of strong secrecy for security protocols. In IEEE Symposium on Security and Privacy, pages 86-100, May 2004.

[20] B. Blanchet. A computationally sound mechanized prover for security protocols. Cryptology ePrint Archive, Report 2005/401, Nov. 2005. Available at http://eprint. iacr.org/2005/401.

[21] B. Blanchet. A computationally sound mechanized prover for security protocols. In IEEE Symposium on Security and Privacy, pages 140-154, May 2006.

[22] B. Blanchet and D. Pointcheval. Automated security proofs with sequences of games. In CRYPTO'06, volume 4117 of LNCS, pages 537-554. Springer, Aug. 2006.

[23] M. Burrows, M. Abadi, and R. Needham. A logic of authentication. Proceedings of the Royal Society of London A, 426:233-271, 1989.

[24] R. Canetti. Universally composable security: A new paradigm for cryptographic protocols. In FOCS'01, pages 136-145. IEEE, Oct. 2001. An updated version is available at Cryptology ePrint Archive, http://eprint.iacr. org/2000/067.

[25] R. Canetti, L. Cheung, D. Kaynar, M. Liskov, N. Linch, O. Pereira, and R. Segala. Time-bounded task-PIOAs: A framework for analyzing security protocols. In DISC'06, volume 4167 of $L N C S$, pages 238-253. Springer, Sept. 2006.

[26] R. Canetti and J. Herzog. Universally composable symbolic analysis of mutual authentication and key exchange protocols. In TCC'06, volume 3876 of $L N C S$, pages 380 403. Springer, Mar. 2006. Extended version available at http://eprint.iacr.org/2004/334.

[27] R. Canetti and H. Krawczyk. Analysis of key-exchange protocols and their use for building secure channels. In $E U$ ROCRYPT 2001, volume 2045 of LNCS, pages 453-474. Springer, May 2001

[28] R. Corin. A probabilistic Hoare-style logic for game-based cryptographic proofs. In ICALP'06, volume 4052 of $L N C S$, pages 252-263. Springer, July 2006.

[29] V. Cortier, H. Hördegen, and B. Warinschi. Explicit randomness is not necessary when modeling probabilistic encryption. In C. Dima, M. Minea, and F. Tiplea, editors, Workshop on Information and Computer Security (ICS 2006), volume 186 of ENTCS, pages 49-65, Timisoara, Romania, Sept. 2006. Elsevier.

[30] V. Cortier, S. Kremer, R. Küsters, and B. Warinschi. Computationally sound symbolic secrecy in the presence of hash functions. In FSTTCS'06, volume 4246 of $L N C S$, pages 176-187. Springer, Dec. 2006.

[31] V. Cortier and B. Warinschi. Computationally sound, automated proofs for security protocols. In ESOP'05, volume 3444 of LNCS, pages 157-171. Springer, Apr. 2005.

[32] A. Datta, A. Derek, J. C. Mitchell, V. Shmatikov, and M. Turuani. Probabilistic polynomial-time semantics for a protocol security logic. In ICALP'05, volume 3580 of $L N C S$, pages 16-29. Springer, July 2005.

[33] A. Datta, A. Derek, J. C. Mitchell, and B. Warinschi. Computationally sound compositional logic for key exchange protocols. In $C S F W^{\prime} 06$, pages 321-334. IEEE Computer Society, July 2006. 
[34] D. E. Denning and G. M. Sacco. Timestamps in key distribution protocols. Commun. ACM, 24(8):533-536, Aug. 1981.

[35] S. Goldwasser, S. Micali, and R. Rivest. A digital signature scheme secure against adaptative chosen-message attacks. SIAM Journal of Computing, 17(2):281-308, Apr. 1988.

[36] A. Gordon and A. Jeffrey. Authenticity by typing for security protocols. In $C S F W^{\prime} 01$, pages 145-159. IEEE Computer Society, June 2001.

[37] S. Halevi. A plausible approach to computer-aided cryptographic proofs. Cryptology ePrint Archive, Report 2005/181, June 2005. Available at http://eprint. iacr.org/2005/181.

[38] J. Herzog. A computational interpretation of Dolev-Yao adversaries. In WITS'03, pages 146-155, Apr. 2003.

[39] R. Janvier, Y. Lakhnech, and L. Mazaré. Completing the picture: Soundness of formal encryption in the presence of active adversaries. In ESOP'05, volume 3444 of $L N C S$, pages 172-185. Springer, Apr. 2005.

[40] I. R. Jeong, J. Katz, and D. H. Lee. One-round protocols for two-party authenticated key exchange. In Applied Cryptography and Network Security, volume 3089 of LNCS, pages 220-232. Springer, June 2004.

[41] D. E. Knuth and P. B. Bendix. Simple word problems in universal algebras. In Computational Problems in Abstract Algebra, pages 263-297. Pergamon Press, 1970.

[42] H. Krawczyk. HMQV: A high-performance secure DiffieHellman protocol. In CRYPTO'05, volume 3621 of LNCS, pages 546-566. Springer, Aug. 2005.

[43] P. Laud. Handling encryption in an analysis for secure information flow. In ESOP'03, volume 2618 of $L N C S$, pages 159-173. Springer, Apr. 2003.

[44] P. Laud. Symmetric encryption in automatic analyses for confidentiality against active adversaries. In IEEE Symposium on Security and Privacy, pages 71-85, May 2004.

[45] P. Laud. Secrecy types for a simulatable cryptographic library. In $C C S^{\prime} 05$, pages 26-35. ACM, Nov. 2005.

[46] P. D. Lincoln, J. C. Mitchell, M. Mitchell, and A. Scedrov. A probabilistic poly-time framework for protocol analysis. In $C C S^{\prime}$ '98, pages 112-121, Nov. 1998.

[47] P. D. Lincoln, J. C. Mitchell, M. Mitchell, and A. Scedrov. Probabilistic polynomial-time equivalence and security protocols. In FM'99, volume 1708 of LNCS, pages 776-793. Springer, Sept. 1999.

[48] G. Lowe. Breaking and fixing the Needham-Schroeder public-key protocol using FDR. In TACAS'96, volume 1055 of $L N C S$, pages 147-166. Springer, 1996.

[49] P. Mateus, J. Mitchell, and A. Scedrov. Composition of cryptographic protocols in a probabilistic polynomial-time process calculus. In CONCUR'03, volume 2761 of $L N C S$, pages 327-349. Springer, Sept. 2003.

[50] D. Micciancio and B. Warinschi. Completeness theorems for the Abadi-Rogaway logic of encrypted expressions. Journal of Computer Security, 12(1):99-129, 2004.

[51] D. Micciancio and B. Warinschi. Soundness of formal encryption in the presence of active adversaries. In $T C C^{\prime} 04$, volume 2951 of $L N C S$, pages 133-151. Springer, Feb. 2004.
[52] J. C. Mitchell, A. Ramanathan, A. Scedrov, and V. Teague. A probabilistic polynomial-time calculus for the analysis of cryptographic protocols. Theoretical Computer Science, 353(1-3):118-164, Mar. 2006.

[53] R. M. Needham and M. D. Schroeder. Using encryption for authentication in large networks of computers. Commun. ACM, 21(12):993-999, Dec. 1978.

[54] R. M. Needham and M. D. Schroeder. Authentication revisited. Operating Systems Review, 21(1):7, 1987.

[55] D. Otway and O. Rees. Efficient and timely mutual authentication. Operating Systems Review, 21(1):8-10, 1987.

[56] A. Ramanathan, J. Mitchell, A. Scedrov, and V. Teague. Probabilistic bisimulation and equivalence for security analysis of network protocols. In FOSSACS'04, volume 2987 of LNCS, pages 468-483. Springer, Mar. 2004.

[57] V. Shoup. A proposal for an ISO standard for public-key encryption, Dec. 2001. ISO/IEC JTC 1/SC27.

[58] V. Shoup. OAEP reconsidered. Journal of Cryptology, 15(4):223-249, Sept. 2002.

[59] V. Shoup. Sequences of games: a tool for taming complexity in security proofs. Cryptology ePrint Archive, Report 2004/332, Nov. 2004. Available at http: / / eprint. iacr.org/2004/332.

[60] C. Sprenger, M. Backes, D. Basin, B. Pfitzmann, and M. Waidner. Cryptographically sound theorem proving. In CSFW'06, pages 153-166. IEEE, July 2006.

[61] S. Tarento. Machine-checked security proofs of cryptographic signature schemes. In ESORICS'05, volume 3679 of LNCS, pages 140-158. Springer, Sept. 2005.

[62] T. Y. C. Woo and S. S. Lam. Authentication for distributed systems. Computer, 25(1):39-52, Jan. 1992.

[63] T. Y. C. Woo and S. S. Lam. A semantic model for authentication protocols. In Proceedings IEEE Symposium on Research in Security and Privacy, pages 178-194, May 1993.

[64] T. Y. C. Woo and S. S. Lam. Authentication for distributed systems. In Internet Besieged: Countering Cyberspace Scofflaws, pages 319-355. ACM Press and Addison-Wesley, Oct. 1997.

\section{Appendix}

Appendices A, B, and C should be read in this order, because Appendices A and B introduce notations and results used in the proofs in Appendix C.

\section{A. Additional Information on the Calculus}

The full syntax of our calculus is given in Figure 1. This calculus distinguishes two categories of processes: input processes wait for a message on a channel; output processes execute some internal computations and output the result on a channel. Most constructs have already been explained in Section 2. We complement these explanations here. The nil process 0 does nothing. The find construct may have several branches: find $\left(\bigoplus_{j=1}^{m} u_{j 1} \widetilde{[i]} \leq n_{j 1}, \ldots, u_{j m_{j}} \widetilde{[i]} \leq n_{j m_{j}}\right.$ suchthat defined $\left(M_{j 1}, \ldots, M_{j l_{j}}\right) \wedge M_{j}$ then $\left.P_{j}\right)$ else $P$ 
tries to find a branch $j$ in $[1, m]$ such that there are values of $u_{j 1}, \ldots, u_{j m_{j}}$ for which $M_{j 1}, \ldots, M_{j l_{j}}$ are defined and $M_{j}$ is true. In case of success, it executes $P_{j}$. (If there are several successful choices of $j, u_{j 1}, \ldots, u_{j m_{j}}$, one of them is chosen randomly with uniform probability.) In case of failure for all branches, it executes $P$. The conditional if defined $\left(M_{1}, \ldots, M_{l}\right) \wedge M$ then $P$ else $P^{\prime}$ is defined as syntactic sugar for find suchthat defined $\left(M_{1}, \ldots, M_{l}\right) \wedge M$ then $P$ else $P^{\prime}$. The conjunct defined $\left(M_{1}, \ldots, M_{l}\right)$ can be omitted when $l=0$ and $M$ can be omitted when it is true. An else branch of find or if may be omitted when it is else $\overline{\text { yield }}\langle\rangle ; 0$. (Note that "else 0 " would not be syntactically correct.) A trailing 0 after an output may be omitted.

The semantics of the calculus is formally defined as a probabilistic reduction relation on semantic configurations $\mathbb{C}$. A semantic configuration $\mathbb{C}$ is a quadruple $E,(\sigma, P), \mathcal{Q}, \mathcal{C}$, where

- $E$ is an environment that maps array cells to bitstrings or $\perp$,

- $P$ is the output process currently scheduled and $\sigma$ is a mapping of the replication indices at $P$ to integers,

- $\mathcal{Q}$ is a multiset of pairs $\left(\sigma^{\prime}, Q\right)$ where the $Q$ 's are input processes currently waiting for messages and $\sigma^{\prime}$ is a mapping of the replication indices at $Q$ to integers,

- $\mathcal{C}$ is the set of channels already created.

The semantics is defined by reduction rules of the form $E,(\sigma, P), \mathcal{Q}, \mathcal{C} \quad \stackrel{[e]}{\longrightarrow}_{p, t} \quad E^{\prime},\left(\sigma^{\prime}, P^{\prime}\right), \mathcal{Q}^{\prime}, \mathcal{C}^{\prime}$ meaning that $E,(\sigma, P), \mathcal{Q}, \mathcal{C}$ reduces to $E^{\prime},\left(\sigma^{\prime}, P^{\prime}\right), \mathcal{Q}^{\prime}, \mathcal{C}^{\prime}$ with probability $p$. The label $[e]$ is empty for all reductions, except events, in which case it records the executed event $e\left(a_{1}, \ldots, a_{m}\right)$. The tag $t$ just serves in distinguishing reductions that yield the same configuration with the same probability in different ways, so that the probability of a certain reduction can be computed correctly. (Although the semantics depends on the security parameter $\eta$, its value is omitted to lighten the notation.)

The semantics uses the relation $E, \sigma, M \Downarrow a$, which means that the term $M$ evaluates to the bitstring $a$ in the environments $E$ (which gives values of arrays) and $\sigma$ (which gives values of replication indices).

The semantic rule for events is the following:

$$
\begin{gathered}
\forall j \leq m, E, \sigma, M_{j} \Downarrow a_{j} \\
E,\left(\sigma, \text { event } e\left(M_{1}, \ldots, M_{m}\right) ; P\right), \mathcal{Q}, \mathcal{C} \\
\stackrel{e\left(a_{1}, \ldots, a_{m}\right)}{\longrightarrow} 1, E v \\
\quad E,(\sigma, P), \mathcal{Q}, \mathcal{C}
\end{gathered}
$$

(Event)

The process evaluates the terms $M_{1}, \ldots, M_{m}$ to bitstrings $a_{1}, \ldots, a_{m}$, and executes the event $e\left(a_{1}, \ldots, a_{m}\right)$. This execution is recorded on the label of the transition, and the

$$
\begin{aligned}
& M, N::= \\
& i \quad \text { replication index } \\
& x\left[M_{1}, \ldots, M_{m}\right] \quad \text { variable access } \\
& f\left(M_{1}, \ldots, M_{m}\right) \quad \text { function application } \\
& Q::= \\
& 0 \text { nil } \\
& Q \mid Q^{\prime} \quad \text { parallel composition } \\
& !^{i \leq n} Q \quad \text { replication } n \text { times } \\
& \text { newChannel } c ; Q \quad \text { channel restriction } \\
& c\left[M_{1}, \ldots, M_{l}\right]\left(x_{1} \widetilde{[i]}: T_{1}, \ldots, x_{k} \widetilde{[i]}: T_{k}\right) ; P \\
& \text { input } \\
& P::=\quad \text { output process } \\
& \overline{c\left[M_{1}, \ldots, M_{l}\right]}\left\langle N_{1}, \ldots, N_{k}\right\rangle ; Q \text { output } \\
& \text { new } x \widetilde{[i]}: T ; P \quad \text { random number } \\
& \text { let } x \widetilde{[i]}: T=M \text { in } P \quad \text { assignment } \\
& \text { if defined }\left(M_{1}, \ldots, M_{l}\right) \wedge M \text { then } P \text { else } P^{\prime} \\
& \text { conditional } \\
& \text { find }\left(\bigoplus_{j=1}^{m} u_{j 1} \widetilde{[i]} \leq n_{j 1}, \ldots, u_{j m_{j}} \widetilde{[i]} \leq n_{j m_{j}}\right. \\
& \text { suchthat defined } \left.\left(M_{j 1}, \ldots, M_{j l_{j}}\right) \wedge M_{j} \text { then } P_{j}\right) \\
& \text { else } P \\
& \text { event } e\left(M_{1}, \ldots, M_{m}\right) ; P \quad \text { event }
\end{aligned}
$$

\section{Figure 1. Syntax of the process calculus}

event instruction disappears from the process. The probability of this transition is 1 and its tag is $E v$.

The other semantic rules are the ones of [20], except for minor changes of notations. ([20] used $\stackrel{p}{\rightarrow}_{\eta}$ instead of $\stackrel{[e]}{\longrightarrow} p, t$ because there was no event. The processes were directly instantiated with the values of the replication indices, so that the semantics of [20] used $\sigma P$ where this paper uses $(\sigma, P)$.)

The initial configuration for running process $Q_{0}$ is $\operatorname{initConfig}\left(Q_{0}\right)=\emptyset,\left(\sigma_{0}, \overline{\text { start }}\langle\rangle\right),\left\{\left(\sigma_{0}, Q_{0}\right)\right\}, \operatorname{fc}\left(Q_{0}\right)$ where $\sigma_{0}$ is the function defined nowhere. Hence, the process begins with sending an empty message on channel start. The process $Q_{0}$ should wait for a message on that channel. We denote a trace of $Q_{0}$ by initConfig $\left(Q_{0}\right) \stackrel{\mathcal{E}}{\rightarrow} p, \mathcal{T}$ $E,(\sigma, P), \mathcal{Q}, \mathcal{C}$ where $p>0$ is the probability of this trace and $\mathcal{T}$ is a sequence of tags that determine the transitions (one tag per transition).

The following two properties are easy to prove from the definition of the semantics:

Proposition 5 If initConfig $\left(Q_{0}\right) \stackrel{\mathcal{E}}{\rightarrow} p, \mathcal{T} E,(\sigma, P), \mathcal{Q}, \mathcal{C}$, then $P$ is a subprocess of $Q_{0}$ or of start \langle\rangle .0 .

Proposition 6 If $E,(\sigma, P), \mathcal{Q}, \mathcal{C} \stackrel{\mathcal{E}}{\rightarrow}_{p, \mathcal{T}} E^{\prime},\left(\sigma^{\prime}, P^{\prime}\right), \mathcal{Q}^{\prime}$, $\mathcal{C}^{\prime}$, then $E^{\prime}$ is an extension of $E$. 


\section{B. Proof Engine}

In this section, we define the proof engine that our tool uses for reasoning on games. This engine is used both for simplifying games and for proving correspondences. The version presented here is slightly simplified; the full version can be found in [20]. Our proof engine uses both equations given by the user, that come in particular from algebraic properties of cryptographic primitives, and facts that hold at certain points in the game due to the form of the game. The engine uses this information in order to infer equalities using a Knuth-Bendix-like equational prover.

\section{B.1. User-defined Rewrite Rules}

The user can give properties of the form $\forall x_{1}: T_{1}, \ldots$, $\forall x_{m}: T_{m}, M$ which mean that, for all environments $\rho$ that map variables to bitstrings, if for all $j \leq m, \rho\left(x_{j}\right) \in$ $I_{\eta}\left(T_{j}\right)$, then $\rho, M \Downarrow$ true. lows:

These properties are translated into rewrite rules as fol-

- If $M$ is of the form $M_{1}=M_{2}$ and $\operatorname{var}\left(M_{2}\right) \subseteq$ $\operatorname{var}\left(M_{1}\right)$, we generate the rewrite rule $\forall x_{1}: T_{1}, \ldots$, $\forall x_{m}: T_{m}, M_{1} \rightarrow M_{2}$.

- If $M$ is of the form $M_{1} \neq M_{2}$, we generate the rewrite rules $\forall x_{1}: T_{1}, \ldots, \forall x_{m}: T_{m},\left(M_{1}=M_{2}\right) \rightarrow$ false, $\forall x_{1}: T_{1}, \ldots, \forall x_{m}: T_{m},\left(M_{1} \neq M_{2}\right) \rightarrow$ true. (Such rules are used for instance to express that different constants are different.)

- Otherwise, we generate the rewrite rule $\forall x_{1}: T_{1}, \ldots$, $\forall x_{m}: T_{m}, M \rightarrow$ true.

The term $M$ reduces into $M^{\prime}$ by the rewrite rule $\forall x_{1}: T_{1}$, $\ldots, \forall x_{m}: T_{m}, M_{1} \rightarrow M_{2}$ if and only if $M=C\left[\theta M_{1}\right]$, $M^{\prime}=C\left[\theta M_{2}\right]$, where $C$ is a term context and $\theta$ is a substitution that maps $x_{j}$ to any term of type $T_{j}$ for all $j \leq m$.

The prover has built-in rewrite rules for defining boolean functions:

$$
\begin{aligned}
& \neg \text { true } \rightarrow \text { false } \quad \neg \text { false } \rightarrow \text { true } \\
& \forall x: \text { bool, } \neg(\neg x) \rightarrow x \\
& \forall x: T, \forall y: T, \neg(x=y) \rightarrow x \neq y \\
& \forall x: T, \forall y: T, \neg(x \neq y) \rightarrow x=y \\
& \forall x: T, x=x \rightarrow \text { true } \quad \forall x: T, x \neq x \rightarrow \text { false } \\
& \forall x: \text { bool, } \forall y: \text { bool, } \neg(x \wedge y) \rightarrow(\neg x) \vee(\neg y) \\
& \forall x: \text { bool, } \forall y: \text { bool, } \neg(x \vee y) \rightarrow(\neg x) \wedge(\neg y) \\
& \forall x: \text { bool, } x \wedge \text { true } \rightarrow x \quad \forall x: \text { bool, } x \wedge \text { false } \rightarrow \text { false } \\
& \forall x: \text { bool, } x \vee \text { true } \rightarrow \text { true } \quad \forall x: \text { bool, } x \vee \text { false } \rightarrow x
\end{aligned}
$$

The prover also has support for commutative function symbols. For such symbols, all equality and matching tests are performed modulo commutativity. The functions $\wedge, \vee$, $=$, and $\neq$ are commutative. So, for instance, the last four rewrite rules above may also be used to rewrite true $\wedge M$ into $M$, false $\wedge M$ into false, true $\vee M$ into true, and false $\vee$ $M$ into $M$.

\section{B.2. Collecting True Facts from a Game}

The function collectFacts collects facts defined $(M)$, event $\left(e\left(M_{1}, \ldots, M_{m}\right)\right)$, and terms $M$ that hold at each program point of the game. More precisely, for each occurrence $P$ of a subprocess of the game, it computes a set $\mathcal{F}_{P}$ of facts that hold at that occurrence. (It is important that $P$ is an occurrence and not a process: processes at several occurrences may be equal, and must be distinguished from one another here.) The function collectFacts also computes a set $\mathcal{D}$ containing pairs $(x \widetilde{i}], P)$ where $x \widetilde{[i]}$ has been defined just above process $P$. (If there are several definitions of $x$, there is one such pair for each definition of $x$.) Finally, for output processes $P$, $\operatorname{collectFacts}(P)$ returns a set of facts that will hold when the next output is executed, and stores this set in $\mathcal{F}_{P}^{\text {Fut }}$. (The superscript Fut stands for future, since these facts do not hold yet at $P$, but will hold in the future.)

The function collectFacts is defined in Figure 2. It is initially called by collectFacts $\left(Q_{0}\right)$. It takes into account that $x \widetilde{[i]}$ may be defined by an input, a restriction, a let, or a find, and updates $\mathcal{D}$ accordingly. Furthermore, when we execute let $x \widetilde{i}]: T=M$ in $\left.P^{\prime}, x \widetilde{x}\right]=M$ holds in $P^{\prime}$ and $x[\widetilde{i}]$ is defined in $P^{\prime}$. When we execute find $\left(\bigoplus_{j=1}^{m} u_{j 1} \widetilde{[i]} \leq n_{j 1}, \ldots, u_{j m_{j}} \widetilde{[i]} \leq n_{j m_{j}}\right.$ suchthat defined $\left(M_{j 1}, \ldots, M_{j l_{j}}\right) \wedge M_{j}$ then $\left.P_{j}\right)$ else $P^{\prime}, M_{j}$ holds in $\left.P_{j}, M_{j 1}, \ldots, M_{j l_{j}}, u_{j 1}[\widetilde{i}], \ldots, u_{j m_{j}} \widetilde{[i}\right]$ are defined in $P_{j}$, and $\neg M_{j}$ holds in $P^{\prime}$ when $m_{j}=l_{j}=0$. When we execute event $e\left(M_{1}, \ldots, M_{n}\right)$, that execution is recorded by a fact event $\left(e\left(M_{1}, \ldots, M_{n}\right)\right)$.

After calling collectFacts $\left(Q_{0}\right)$, we complete the computed sets $\mathcal{F}_{P}$ (where $P$ may be an input or output process) by adding facts that come from processes above $P$ :

$$
\mathcal{F}_{P} \leftarrow \mathcal{F}_{P} \cup \mathcal{F}_{P^{\prime}} \text { if } P \text { is immediately under } P^{\prime}
$$

We also add facts that we can deduce from facts defined $(M)$. Precisely, if defined $(M) \in \mathcal{F}_{P}$, and $x\left[M_{1}\right.$, $\left.\ldots, M_{m}\right]$ is a subterm of $M$, we take into account facts that are known to be true at the definitions of $x$ by adding them to $\mathcal{F}_{P}$ as follows:

$$
\begin{aligned}
\mathcal{F}_{P} \leftarrow \mathcal{F}_{P} \cup & \left\{\begin{array}{r}
\sigma\left(\mathcal{F}_{P^{\prime}} \cup\left(\mathcal{F}_{P^{\prime}}^{\text {Fut }} \cap \mathcal{F}_{P}\right)\right) \\
\text { if } P \text { is under } P^{\prime} \\
\sigma\left(\mathcal{F}_{P^{\prime}} \cup \mathcal{F}_{P^{\prime}}^{\text {Fut }}\right) \text { otherwise }
\end{array}\right)
\end{aligned}
$$


collectFacts $(Q)=$

if $Q=Q_{1} \mid Q_{2}$ then $\operatorname{collectFacts}\left(Q_{1}\right) ; \operatorname{collectFacts}\left(Q_{2}\right)$

if $Q=!^{i \leq n} Q^{\prime}$ then collectFacts $\left(Q^{\prime}\right)$

if $Q=$ newChannel $c ; Q^{\prime}$ then collectFacts $\left(Q^{\prime}\right)$

if $\left.\left.Q=c\left[M_{1}, \ldots, M_{l}\right]\left(x_{1} \widetilde{[}\right]: T_{1}, \ldots, x_{k} \widetilde{i}\right]: T_{k}\right) ; P$ then

$\mathcal{F}_{P}=\left\{\operatorname{defined}\left(x_{j}[\widetilde{i}]\right) \mid j \leq k\right\} ;$

$\mathcal{F}_{P}^{\text {Fut }}=\operatorname{collectFacts}(P)$

$\left.\mathcal{D}=\mathcal{D} \cup\left\{\left(x_{j} \widetilde{[i}\right], P\right) \mid j \leq k\right\}$

$\operatorname{collectFacts}(P)=$

if $P=\overline{c\left[M_{1}, \ldots, M_{l}\right]}\left\langle N_{1}, \ldots, N_{k}\right\rangle ; Q$ then

collectFacts $(Q)$; return $\emptyset$

if $P=$ new $x[\tilde{i}]: T ; P^{\prime}$ then

$\left.\mathcal{F}_{P^{\prime}}=\{\operatorname{defined}(x \widetilde{[i}])\right\} ; \mathcal{F}_{P^{\prime}}^{\text {Fut }}=\operatorname{collectFacts}\left(P^{\prime}\right)$

$\left.\mathcal{D}=\mathcal{D} \cup\left\{(x \widetilde{i}], P^{\prime}\right)\right\} ;$ return $\mathcal{F}_{P^{\prime}} \cup \mathcal{F}_{P^{\prime}}^{\text {Fut }}$

if $P=$ let $x[\widetilde{i}]: T=M$ in $P^{\prime}$ then

$\mathcal{F}_{P^{\prime}}=\{\operatorname{defined}(x[\widetilde{i}]), x[\widetilde{i}]=M\}$

$\mathcal{F}_{P^{\prime}}^{\text {Fut }}=\operatorname{collectFacts}\left(P^{\prime}\right)$

$\left.\mathcal{D}=\mathcal{D} \cup\left\{(x \widetilde{i}], P^{\prime}\right)\right\} ;$ return $\mathcal{F}_{P^{\prime}} \cup \mathcal{F}_{P^{\prime}}^{\text {Fut }}$

if $P=$ find $\left(\bigoplus_{j=1}^{m} u_{j 1} \widetilde{[i]} \leq n_{j 1}, \ldots, u_{j m_{j}} \widetilde{i}\right] \leq n_{j m_{j}}$

suchthat defined $\left(M_{j 1}, \ldots, M_{j l_{j}}\right) \wedge M_{j}$ then $\left.P_{j}\right)$ else $P^{\prime}$ then

for each $j \leq m$,

$$
\mathcal{F}_{P_{j}}=\left\{\operatorname{defined}\left(u_{j 1}\left[\tilde{i^{\prime}}\right]\right), \ldots, \operatorname{defined}\left(u_{j m_{j}}\left[\widetilde{i^{\prime}}\right]\right),\right.
$$$$
\text { defined } \left.\left(M_{j 1}\right), \ldots, \text { defined }\left(M_{j l_{j}}\right), M_{j}\right\}
$$

$\mathcal{F}_{P_{j}}^{\text {Fut }}=\operatorname{collectFacts}\left(P_{j}\right)$;

$\mathcal{D}=\mathcal{D} \cup\left\{\left(u_{j 1}\left[\widetilde{i^{\prime}}\right], P_{j}\right), \ldots,\left(u_{j m_{j}}\left[\widetilde{i^{\prime}}\right], P_{j}\right)\right\}$

$\mathcal{F}_{P^{\prime}}=\left\{\neg M_{j} \mid m_{j}=l_{j}=0\right\} ;$

$\mathcal{F}_{P^{\prime}}^{\text {Fut }}=\operatorname{collectFacts}\left(P^{\prime}\right)$

$\operatorname{return}\left(\mathcal{F}_{P^{\prime}} \cup \mathcal{F}_{P^{\prime}}^{\text {Fut }}\right) \cap \bigcap_{j=1}^{m}\left(\mathcal{F}_{P_{j}} \cup \mathcal{F}_{P_{j}}^{\text {Fut }}\right)$

if $P=$ event $e\left(M_{1}, \ldots, M_{n}\right) ; P^{\prime}$ then

$\mathcal{F}_{P^{\prime}}=\left\{\operatorname{event}\left(e\left(M_{1}, \ldots, M_{n}\right)\right)\right\}$

$\operatorname{collectFacts}\left(P^{\prime}\right)$

Figure 2. The function collectFacts where $\sigma=\left\{M_{1} / i_{1}, \ldots, M_{m} / i_{m}\right\}$. Indeed, if defined $(M) \in \mathcal{F}_{P}$, and $x\left[M_{1}, \ldots, M_{m}\right]$ is a subterm of $M$, then $x\left[M_{1}, \ldots, M_{m}\right]$ is defined at $P$, so some definition of $x\left[M_{1}, \ldots, M_{m}\right]$, just above the process $P^{\prime}$, must have been executed before reaching $P$, so the facts that hold at $P^{\prime}$ also hold at $P$, with a suitable substitution of indices: we have $\sigma \mathcal{F}_{P^{\prime}}$, that is, $\mathcal{F}_{P^{\prime}}\left\{M_{1} / i_{1}, \ldots, M_{m} / i_{m}\right\}$. Moreover, if the occurrence $P$ is not syntactically under the occurrence $P^{\prime}$, then the code of $P^{\prime}$ must have been executed until the next output before executing some other code and reaching $P$, so in fact $\sigma\left(\mathcal{F}_{P^{\prime}} \cup \mathcal{F}_{P^{\prime}}^{\text {Fut }}\right)$ hold. If $P$ is syntactically under $P^{\prime}$, it is possible that the code of $P^{\prime}$ has been executed until reaching $P$ instead of until reaching the next output, so we have only $\sigma\left(\mathcal{F}_{P^{\prime}} \cup\left(\mathcal{F}_{P^{\prime}}^{\text {Fut }} \cap \mathcal{F}_{P}\right)\right)$. If there are several definitions of $x$, we do not know which one has been executed, so we only add to $\mathcal{F}_{P}$ the facts that hold in all cases, by taking the intersection on all definitions of $x$.

This operation may add new defined facts to $\mathcal{F}_{P}$, so it is executed until a fixpoint is reached, except that, in order to avoid infinite loops, we do not execute this step for definitions defined $(M)$ in which $M$ contains nested occurrences of the same symbol (such as $x[\ldots x[\ldots] \ldots]$ ).

We formally define the semantics of facts as follows: $E, \sigma, \mathcal{E} \vdash F$ when the fact $F$ holds in the environments $E$ and $\sigma$ for the sequence of events $\mathcal{E}$.

$E, \sigma, \mathcal{E} \vdash M$ if and only if $E, \sigma, M \Downarrow$ true

$E, \sigma, \mathcal{E} \vdash \operatorname{defined}(M)$ if and only if

$E, \sigma, M \Downarrow a$ for some $a$

$E, \sigma, \mathcal{E} \vdash \operatorname{event}\left(e\left(M_{1}, \ldots, M_{m}\right)\right)$ if and only if

there exist $a_{1}, \ldots, a_{m}$ such that for all $j \leq m$,

$E, \sigma, M_{j} \Downarrow a_{j}$ and $e\left(a_{1}, \ldots, a_{m}\right) \in \mathcal{E}$

We extend this definition to formulae built from facts by conjunctions and disjunctions:

$E, \rho, \mathcal{E} \vdash \phi_{1} \wedge \phi_{2}$ if and only if

$E, \rho, \mathcal{E} \vdash \phi_{1}$ and $E, \rho, \mathcal{E} \vdash \phi_{2}$

$E, \rho, \mathcal{E} \vdash \phi_{1} \vee \phi_{2}$ if and only if

$E, \rho, \mathcal{E} \vdash \phi_{1}$ or $E, \rho, \mathcal{E} \vdash \phi_{2}$

We also extend it naturally to sets of facts and formulae: $E, \sigma, \mathcal{E} \vdash \mathcal{F}$ if and only if for all $F \in \mathcal{F}, E, \sigma, \mathcal{E} \vdash F$.

The following proposition expresses the correctness of the collection of true facts. A detailed proof of this result for the full algorithm used in the implementation, but for the version without events, can be found in [20]. The extension to events is straightforward.

Proposition 7 If initConfig $\left(C\left[Q_{0}\right]\right) \stackrel{\mathcal{E}}{\rightarrow}_{p, \mathcal{T}} E,(\sigma, P), \mathcal{Q}$, $\mathcal{C}$, then $E, \sigma, \mathcal{E} \vdash \mathcal{F}_{P}$.

\section{B.3. Equational Prover}

We use an algorithm inspired by the Knuth-Bendix completion algorithm [41], with differences detailed below. 
The prover manipulates pairs $\mathcal{F}, \mathcal{R}$ where $\mathcal{F}$ is a set of facts $\left(M\right.$, defined $(M)$, or event $\left.\left(e\left(M_{1}, \ldots, M_{n}\right)\right)\right)$ and $\mathcal{R}$ is a set of rewrite rules $M_{1} \rightarrow M_{2}$. We say that $M$ reduces into $M^{\prime}$ by $M_{1} \rightarrow M_{2}$ when $M=C\left[M_{1}\right]$ and $M^{\prime}=C\left[M_{2}\right]$ for some term context $C$. (That is, all variables in rewrite rules of $\mathcal{R}$ are considered as constants.) The prover starts with a certain set of facts $\mathcal{F}$ and $\mathcal{R}=\emptyset$. Then the prover transforms the pairs $(\mathcal{F}, \mathcal{R})$ by the following rules (the rule $\frac{\mathcal{F}, \mathcal{R}}{\mathcal{F}^{\prime}, \mathcal{R}^{\prime}}$ means that $\mathcal{F}, \mathcal{R}$ is transformed into $\mathcal{F}^{\prime}, \mathcal{R}^{\prime}$ ):

$\frac{\mathcal{F} \cup\{F\}, \mathcal{R}}{\mathcal{F} \cup\left\{F^{\prime}\right\}, \mathcal{R}} \quad$ if $F$ reduces into $F^{\prime}$ by a rule of

$\overline{\mathcal{F} \cup\left\{F^{\prime}\right\}, \mathcal{R}} \quad \mathcal{R}$ or a user-defined rewrite rule $\frac{\mathcal{F} \cup\left\{M_{1} \wedge M_{2}\right\}, \mathcal{R}}{\mathcal{F} \cup\left\{M_{1}, M_{2}\right\}, \mathcal{R}}$

$\frac{\mathcal{F} \cup\left\{x\left[M_{1}, \ldots, M_{m}\right]=x\left[M_{1}^{\prime}, \ldots, M_{m}^{\prime}\right]\right\}, \mathcal{R}}{\mathcal{F} \cup\left\{M_{1}=M_{1}^{\prime}, \ldots, M_{m}=M_{m}^{\prime}\right\}, \mathcal{R}}$ when $x$ is defined only by restrictions new $x: T$ and $T$ is a large type

$$
\frac{\mathcal{F} \cup\left\{M=M^{\prime}\right\}, \mathcal{R}}{\mathcal{F}, \mathcal{R} \cup\left\{M \rightarrow M^{\prime}\right\}} \text { if } M>M^{\prime}
$$

$\frac{\mathcal{F}, \mathcal{R} \cup\left\{M_{1} \rightarrow M_{2}\right\}}{\mathcal{F} \cup\left\{M_{1}=M_{2}^{\prime}\right\}, \mathcal{R}} \quad \begin{aligned} & \text { if } M_{2} \text { reduces into } M_{2}^{\prime} \text { by } \\ & \text { user-defined rewrite rule }\end{aligned}$

$\frac{\mathcal{F}, \mathcal{R} \cup\left\{M_{1} \rightarrow M_{2}\right\}}{\mathcal{F} \cup\left\{M_{1}^{\prime}=M_{2}\right\}, \mathcal{R}} \quad \begin{aligned} & \text { if } M_{1} \text { reduces into of } \mathcal{R}\end{aligned}$

We also use the symmetric of Rule (11) obtained by swapping the two sides of the equality.

Rule (8) simplifies facts using rewrite rules. Rule (9) decomposes conjunctions of facts. Rule (10) exploits the elimination of collisions between random values. It takes into account that, when $x$ is defined by a restriction of a large type, two different cells of $x$ have a negligible probability of containing the same value. So when two cells of $x$ contain the same value, we can conclude up to negligible probability that they are the same cell.

Rule (11) is applied only when Rules (8) to (10) cannot be applied. Rule (11) transforms equations into rewrite rules by orienting them. We say that $M>M^{\prime}$ when either $M$ is the form $x[\widetilde{M}], x$ does not occur in $M^{\prime}$, and $x$ is not defined only by restrictions, or $M=x\left[M_{1}, \ldots, M_{m}\right]$, $M^{\prime}=x\left[M_{1}^{\prime}, \ldots, M_{m}^{\prime}\right]$, and for all $j \leq m, M_{j}>M_{j}^{\prime}$. Intuitively, our goal is to replace $M$ with $M^{\prime}$ when $M^{\prime}$ defines the content of the variable $M$. (Notice that this is not an ordering; the Knuth-Bendix algorithm normally uses a reduction ordering to orient equations. However, we tried some reduction orderings, namely the lexicographic path ordering and the Knuth-Bendix ordering, and obtained disappointing results: the prover fails to prove many equalities because too many equations are left unoriented. The simple heuristic given above succeeds more often, at the expense of a greater risk of non-termination, but that does not cause problems in practice on our examples. We believe that this comes from the particular structure of equations, which come from let definitions and from conditions of find or if, and tend to define variables from other variables without creating dependency cycles.)

Rules (12) and (13) are systematically applied to simplify all rewrite rules of $\mathcal{R}$ after a new rewrite rule has been added by Rule (11). Since all terms in rewrite rules of $\mathcal{R}$ are considered as constants, Rule (13) in fact includes the deduction of equations from critical pairs done by the standard Knuth-Bendix completion algorithm.

We say that $\mathcal{F}$ yields a contradiction when the prover starting from $(\mathcal{F}, \emptyset)$ derives $\left(\mathcal{F}^{\prime}, \mathcal{R}^{\prime}\right)$ with false $\in \mathcal{F}^{\prime}$.

We write $E, \rho, \mathcal{E} \vdash \mathcal{F}, \mathcal{R}$ when $E, \rho, \mathcal{E} \vdash \mathcal{F}$ and for all $M_{1} \rightarrow M_{2} \in \mathcal{R}, E, \rho, \mathcal{E} \vdash M_{1}=M_{2}$. A variant of the following result is proved in [20]. This result shows the soundness of the transformation of $\mathcal{F}, \mathcal{R}$ into $\mathcal{F}^{\prime}, \mathcal{R}^{\prime}$ for each rule $\frac{\mathcal{F}, \mathcal{R}}{\mathcal{F}^{\prime}, \mathcal{R}^{\prime}}$ of the equational prover.

Proposition 8 If $\frac{\mathcal{F}, \mathcal{R}}{\mathcal{F}^{\prime}, \mathcal{R}^{\prime}}$, then $\operatorname{Pr}[\exists(E, \sigma, P, \mathcal{Q}, \mathcal{C}, \rho, \mathcal{E})$, $\operatorname{initConfig}\left(C\left[Q_{0}\right]\right) \stackrel{\mathcal{E}}{\rightarrow} E,(\sigma, P), \mathcal{Q}, \mathcal{C} \wedge E, \rho, \mathcal{E} \vdash \mathcal{F}, \mathcal{R} \wedge$ $\left.\neg E, \rho, \mathcal{E} \vdash \mathcal{F}^{\prime}, \mathcal{R}^{\prime}\right]$ is negligible.

We denote by $\operatorname{Pr}\left[C\left[Q_{0}\right] \rightsquigarrow \mathcal{F}\right]$ the probability that $C\left[Q_{0}\right]$ reduces into a configuration in which $\mathcal{F}$ holds: $\operatorname{Pr}\left[C\left[Q_{0}\right] \rightsquigarrow \mathcal{F}\right]=\operatorname{Pr}[\exists(E, \sigma, P, \mathcal{Q}, \mathcal{C}, \rho, \mathcal{E})$, $\left.\operatorname{initConfig}\left(C\left[Q_{0}\right]\right) \stackrel{\mathcal{E}}{\rightarrow} E,(\sigma, P), \mathcal{Q}, \mathcal{C} \wedge E, \rho, \mathcal{E} \vdash \mathcal{F}\right]$.

Proposition 9 If $\mathcal{F}$ yields a contradiction, then $\operatorname{Pr}\left[C\left[Q_{0}\right]\right.$ $\rightsquigarrow \mathcal{F}$ ] is negligible.

Proof This is an easy consequence of Proposition 8 . Since $\mathcal{F}$ yields a contradiction, the prover transforms $(\mathcal{F}, \mathcal{R})=(\mathcal{F}, \emptyset)$ into $\left(\mathcal{F}^{\prime}, \mathcal{R}^{\prime}\right)$ that contains false, so $E, \rho, \mathcal{E} \vdash \mathcal{F}$ implies $E, \rho, \mathcal{E} \vdash \mathcal{F}, \mathcal{R}$, and $\neg E, \rho, \mathcal{E} \vdash$ $\mathcal{F}^{\prime}, \mathcal{R}^{\prime}$. By Proposition 8 applied as many times as there are transformation steps between $(\mathcal{F}, \mathcal{R})$ and $\left(\mathcal{F}^{\prime}, \mathcal{R}^{\prime}\right), \operatorname{Pr}\left[\exists(E, \sigma, P, \mathcal{Q}, \mathcal{C}, \rho, \mathcal{E})\right.$, initConfig $\left(C\left[Q_{0}\right]\right) \stackrel{\mathcal{E}}{\rightarrow}$ $\left.E,(\sigma, P), \mathcal{Q}, \mathcal{C} \wedge E, \rho, \mathcal{E} \vdash \mathcal{F}, \mathcal{R} \wedge \neg E, \rho, \mathcal{E} \vdash \mathcal{F}^{\prime}, \mathcal{R}^{\prime}\right]$ is negligible, which implies that $\operatorname{Pr}\left[C\left[Q_{0}\right] \rightsquigarrow \mathcal{F}\right]$ is negligible.

\section{Proofs}

\section{C.1. Non-injective Correspondences}

The following lemma shows the correctness of $\mathcal{F} \models_{\theta} \phi$, that is, if $\mathcal{F} \Leftrightarrow_{\theta} \phi$, then $\mathcal{F}$ implies $\theta \phi$ with overwhelming probability.

Lemma 3 If $\mathcal{F} \Leftrightarrow_{\theta} \phi$, then $\operatorname{Pr}\left[C\left[Q_{0}\right] \rightsquigarrow \mathcal{F} \cup\{\neg \theta \phi\}\right]$ is negligible. 
Proof The proof proceeds by induction on $\phi$.

- Case $\phi=M$. If $\mathcal{F} \cup\{\neg \theta M\}$ yields a contradiction, then, by Proposition $9, \operatorname{Pr}\left[C\left[Q_{0}\right] \rightsquigarrow \mathcal{F} \cup\{\neg \theta \phi\}\right]$ is negligible.

- Case $\phi=\operatorname{event}\left(e\left(M_{1}, \ldots, M_{m}\right)\right)$. There are terms $M_{1}^{\prime}, \ldots, M_{m}^{\prime}$ such that event $\left(e\left(M_{1}^{\prime}, \ldots, M_{m}^{\prime}\right)\right) \in \mathcal{F}$ and $\mathcal{F} \cup\left\{\bigvee_{j=1}^{m} \theta M_{j} \neq M_{j}^{\prime}\right\}$ yields a contradiction. By Proposition 9, $\operatorname{Pr}\left[C\left[Q_{0}\right] \rightsquigarrow \mathcal{F} \cup\left\{\bigvee_{j=1}^{m} \theta M_{j} \neq M_{j}^{\prime}\right\}\right]$ is negligible. Moreover, if $E, \rho, \mathcal{E} \vdash \mathcal{F} \cup\{\neg \theta \phi\}$, then $E, \rho, \mathcal{E} \vdash \operatorname{event}\left(e\left(M_{1}^{\prime}, \ldots, M_{m}^{\prime}\right)\right)$ and $\neg E, \rho, \mathcal{E} \vdash$ event $\left(e\left(\theta M_{1}, \ldots, \theta M_{m}\right)\right)$, so there exists $j \leq m$ such that $E, \rho, \mathcal{E} \vdash \theta M_{j} \neq M_{j}^{\prime}$, hence $E, \rho, \mathcal{E} \vdash$ $\mathcal{F} \cup\left\{\bigvee_{j=1}^{m} \theta M_{j} \neq M_{j}^{\prime}\right\}$. Therefore, $\operatorname{Pr}\left[C\left[Q_{0}\right] \rightsquigarrow\right.$ $\mathcal{F} \cup\{\neg \theta \phi\}] \leq \operatorname{Pr}\left[C\left[Q_{0}\right] \rightsquigarrow \mathcal{F} \cup\left\{\bigvee_{j=1}^{m} \theta M_{j} \neq M_{j}^{\prime}\right\}\right]$. Hence, $\operatorname{Pr}\left[C\left[Q_{0}\right] \rightsquigarrow \mathcal{F} \cup\{\neg \theta \phi\}\right]$ is negligible.

- Case $\phi=\phi_{1} \wedge \phi_{2}$. We have $\mathcal{F} \Leftrightarrow_{\theta} \phi_{1}$ and $\mathcal{F} \Leftrightarrow_{\theta} \phi_{2}$. By induction hypothesis, $\operatorname{Pr}\left[C\left[Q_{0}\right] \rightsquigarrow \mathcal{F} \cup\left\{\neg \theta \phi_{1}\right\}\right]$ and $\operatorname{Pr}\left[C\left[Q_{0}\right] \rightsquigarrow \mathcal{F} \cup\left\{\neg \theta \phi_{2}\right\}\right]$ are negligible. If $E, \rho, \mathcal{E} \vdash \mathcal{F} \cup\left\{\neg \theta\left(\phi_{1} \wedge \phi_{2}\right)\right\}$, then either $E, \rho, \mathcal{E} \vdash$ $\mathcal{F} \cup\left\{\neg \theta \phi_{1}\right\}$ or $E, \rho, \mathcal{E} \vdash \mathcal{F} \cup\left\{\neg \theta \phi_{2}\right\}$, so $\operatorname{Pr}\left[C\left[Q_{0}\right] \rightsquigarrow\right.$ $\left.\mathcal{F} \cup\left\{\neg \theta\left(\phi_{1} \wedge \phi_{2}\right)\right\}\right] \leq \operatorname{Pr}\left[C\left[Q_{0}\right] \rightsquigarrow \mathcal{F} \cup\left\{\neg \theta \phi_{1}\right\}\right]+$ $\operatorname{Pr}\left[C\left[Q_{0}\right] \rightsquigarrow \mathcal{F} \cup\left\{\neg \theta \phi_{2}\right\}\right]$, so $\operatorname{Pr}\left[C\left[Q_{0}\right] \rightsquigarrow \mathcal{F} \cup\right.$ $\left.\left\{\neg \theta\left(\phi_{1} \wedge \phi_{2}\right)\right\}\right]$ is negligible.

- Case $\phi=\phi_{1} \vee \phi_{2}$. We have either $\mathcal{F} \Leftrightarrow_{\theta} \phi_{1}$ or $\mathcal{F} \Leftrightarrow_{\theta} \phi_{2}$. In the first case, by induction hypothesis, $\operatorname{Pr}\left[C\left[Q_{0}\right] \rightsquigarrow \mathcal{F} \cup\left\{\neg \theta \phi_{1}\right\}\right]$ is negligible. If $E, \rho, \mathcal{E} \vdash$ $\mathcal{F} \cup\left\{\neg \theta\left(\phi_{1} \vee \phi_{2}\right)\right\}$, then $E, \rho, \mathcal{E} \vdash \mathcal{F} \cup\left\{\neg \theta \phi_{1}\right\}$, so $\operatorname{Pr}\left[C\left[Q_{0}\right] \rightsquigarrow \mathcal{F} \cup\left\{\neg \theta\left(\phi_{1} \vee \phi_{2}\right)\right\}\right] \leq \operatorname{Pr}\left[C\left[Q_{0}\right] \rightsquigarrow \mathcal{F} \cup\right.$ $\left.\left\{\neg \theta \phi_{1}\right\}\right]$. Therefore, $\operatorname{Pr}\left[C\left[Q_{0}\right] \rightsquigarrow \mathcal{F} \cup\left\{\neg \theta\left(\phi_{1} \vee \phi_{2}\right)\right\}\right]$ is negligible. The second case follows by symmetry.

Proof of Proposition 1 By hypothesis, if $P_{1}$ follows $F_{1}$, $\ldots$, and $P_{m}$ follows $F_{m}$, then there exists a substitution $\theta$ equal to the identity on the variables of $\psi$ and such that $\mathcal{F}_{F_{1}, P_{1}} \cup \ldots \cup \mathcal{F}_{F_{m}, P_{m}} \Rightarrow_{\theta} \phi$. We let $\theta\left(P_{1}, \ldots P_{m}\right)$ be such a substitution and we define $\mathcal{F}\left(P_{1}, \ldots, P_{m}\right)=\mathcal{F}_{F_{1}, P_{1}} \cup$ $\ldots \cup \mathcal{F}_{F_{m}, P_{m}} \cup\{\neg \theta \phi\}$ where $\theta=\theta\left(P_{1}, \ldots, P_{m}\right)$.

Let $C$ be an evaluation context acceptable for $Q_{0}$ with public variables $V$ that does not contain events used by $\psi \Rightarrow \phi$. Below, we show that if initConfig $\left(C\left[Q_{0}\right]\right) \stackrel{\mathcal{E}}{\rightarrow}_{p, \mathcal{T}}$ $E,(\sigma, P), \mathcal{Q}, \mathcal{C}$ and $\mathcal{E} \forall \psi \Rightarrow \phi$, then there exist $P_{1}$ that follows $F_{1}, \ldots, P_{m}$ that follows $F_{m}$, and $\rho^{\prime}$ such that $E, \rho^{\prime}, \mathcal{E} \vdash \mathcal{F}\left(P_{1}, \ldots, P_{m}\right)$. Therefore,

$$
\begin{aligned}
& \operatorname{Pr}\left[C\left[Q_{0}\right]: \neg(\psi \Rightarrow \phi)\right] \\
& \leq \operatorname{Pr}\left[\begin{array}{l}
\exists(E, \sigma, P, \mathcal{Q}, \mathcal{C}, \mathcal{E}), \\
\operatorname{initConfig}\left(C\left[Q_{0}\right]\right) \stackrel{\mathcal{E}}{\rightarrow} E,(\sigma, P), \mathcal{Q}, \mathcal{C} \wedge \\
\mathcal{E} \nvdash \psi \Rightarrow \phi
\end{array}\right]
\end{aligned}
$$

$$
\begin{aligned}
& \leq \sum_{P_{1}, \ldots, P_{m} \text { that }} \operatorname{Pr}\left[\begin{array}{l}
\exists\left(E, \sigma, P, \mathcal{Q}, \mathcal{C}, \rho^{\prime}, \mathcal{E}\right), \\
\operatorname{initConfig}\left(C\left[Q_{0}\right]\right) \stackrel{\mathcal{E}}{\rightarrow} E,(\sigma, P), \mathcal{Q}, \mathcal{C} \\
\wedge E, \rho^{\prime}, \mathcal{E} \vdash \mathcal{F}\left(P_{1}, \ldots, P_{m}\right)
\end{array}\right] \\
& \text { follow } F_{1}, \ldots, F_{m} \text { respectively } \\
& \leq \sum_{P_{1}, \ldots, P_{m} \text { that follow } F_{1}, \ldots, F_{m} \text { respectively }} \operatorname{Pr}\left[C\left[Q_{0}\right] \rightsquigarrow \mathcal{F}\left(P_{1}, \ldots, P_{m}\right)\right]
\end{aligned}
$$

By Lemma 3, since $\mathcal{F}_{F_{1}, P_{1}} \cup \ldots \cup \mathcal{F}_{F_{m}, P_{m}} \Rightarrow_{\theta} \phi$ for $\theta=$ $\theta\left(P_{1}, \ldots, P_{m}\right)$, the probability $\operatorname{Pr}\left[C\left[Q_{0}\right] \rightsquigarrow \mathcal{F}_{F_{1}, P_{1}} \cup \ldots \cup\right.$ $\left.\mathcal{F}_{F_{m}, P_{m}} \cup\{\neg \theta \phi\}\right]=\operatorname{Pr}\left[C\left[Q_{0}\right] \rightsquigarrow \mathcal{F}\left(P_{1}, \ldots, P_{m}\right)\right]$ is negligible, so the sum is negligible since the number of processes $P_{1}, \ldots, P_{m}$ is independent of the security parameter. Hence, $Q_{0}$ satisfies the correspondence $\psi \Rightarrow \phi$ with public variables $V$.

Assume that initConfig $\left(C\left[Q_{0}\right]\right) \stackrel{\mathcal{E}}{\rightarrow}_{p, \mathcal{T}} E,(\sigma, P), \mathcal{Q}, \mathcal{C}$ and for every $P_{1}$ that follows $F_{1}, \ldots$, for every $P_{m}$ that follows $F_{m}$, for every $\rho^{\prime}$, we have $\neg E, \rho^{\prime}, \mathcal{E} \vdash$ $\mathcal{F}\left(P_{1}, \ldots, P_{m}\right)$. We show that $\mathcal{E} \vdash \psi \Rightarrow \phi$. This result will conclude the proof.

Assume that $\rho, \mathcal{E} \vdash \psi$, where $\rho$ is defined on $\operatorname{var}(\psi)$. For each event $F=\operatorname{event}\left(e\left(M_{1}, \ldots, M_{m^{\prime}}\right)\right)$ in $\psi$, we have $\rho, \mathcal{E} \vdash \operatorname{event}\left(e\left(M_{1}, \ldots, M_{m^{\prime}}\right)\right)$, so for all $j \leq m^{\prime}, \rho, M_{j} \Downarrow a_{j}$ and $e\left(a_{1}, \ldots, a_{m^{\prime}}\right) \in \mathcal{E}$. Since the only transition that produces a label $e\left(a_{1}\right.$, $\left.\ldots, a_{m^{\prime}}\right)$ is (Event), the trace initConfig $\left(Q_{0}\right) \stackrel{\mathcal{E}}{\rightarrow}, \mathcal{T}$ $E,(\sigma, P), \mathcal{Q}, \mathcal{C}$ contains a transition of the form $E^{\prime}$, $\left(\sigma^{\prime}\right.$, event $\left.e\left(M_{1}^{\prime}, \ldots, M_{m^{\prime}}^{\prime}\right) ; P^{\prime}\right), \mathcal{Q}^{\prime}, \mathcal{C}^{\prime} \stackrel{e\left(a_{1}, \ldots, a_{m^{\prime}}\right)}{\longrightarrow}{ }_{1, E v}$ $E^{\prime},\left(\sigma^{\prime}, P^{\prime}\right), \mathcal{Q}^{\prime}, \mathcal{C}^{\prime}$ with $E^{\prime}, \sigma^{\prime}, M_{j}^{\prime} \Downarrow a_{j}$ for all $j \leq m^{\prime}$. By Proposition 5, event $e\left(M_{1}^{\prime}, \ldots, M_{m^{\prime}}^{\prime}\right) ; P^{\prime}$ is a subprocess of $C\left[Q_{0}\right]$ or of $\overline{\text { start }}\langle\rangle ; 0$. Since $C$ does not contain events used by $\psi \Rightarrow \phi$, event $e\left(M_{1}^{\prime}, \ldots, M_{m^{\prime}}^{\prime}\right) ; P^{\prime}$ is a subprocess of $Q_{0}$, so $P^{\prime}$ follows $F$. By Proposition $7, E^{\prime}, \sigma^{\prime}, \mathcal{E}^{\prime} \vdash \mathcal{F}_{P^{\prime}}$, where $\mathcal{E}^{\prime}$ is the prefix of $\mathcal{E}$ until and including the considered occurrence of the event $e\left(a_{1}, \ldots, a_{m^{\prime}}\right)$. By Proposition $6, E$ is an extension of $E^{\prime}$, so $E, \sigma^{\prime}, \mathcal{E} \vdash \mathcal{F}_{P^{\prime}}$. Let $\theta^{\prime}$ be the substitution that renames replication indices at $P^{\prime}$ to fresh replication indices, such that $\mathcal{F}_{F, P^{\prime}}=\theta^{\prime} \mathcal{F}_{P} \cup\left\{\theta^{\prime} M_{j}^{\prime}=M_{j} \mid j \leq m^{\prime}\right\}$. Let $\sigma^{\prime \prime}$ be such that $\sigma^{\prime}=\sigma^{\prime \prime} \theta^{\prime}$. Then $E, \sigma^{\prime \prime}, \mathcal{E} \vdash \theta^{\prime} \mathcal{F}_{P^{\prime}}$. For all $j \leq m^{\prime}$, since $E^{\prime}, \sigma^{\prime}, M_{j}^{\prime} \Downarrow a_{j}$, we have $E, \sigma^{\prime \prime}, \theta^{\prime} M_{j}^{\prime} \Downarrow a_{j}$. We have $\rho, M_{j} \Downarrow a_{j}$. Hence $E, \sigma^{\prime \prime} \oplus \rho, \mathcal{E} \vdash \theta^{\prime} M_{j}^{\prime}=M_{j}$, where $\sigma^{\prime \prime} \oplus \rho$ denotes the function that maps $x$ to $\sigma^{\prime \prime}(x)$ when $x \in \operatorname{Dom}\left(\sigma^{\prime \prime}\right)$ and $i$ to $\rho(i)$ when $i \in \operatorname{Dom}(\rho)$. This function is well defined, since $\operatorname{Dom}\left(\sigma^{\prime \prime}\right)$ and $\operatorname{Dom}(\rho)$ are disjoint. So $E, \sigma^{\prime \prime} \oplus \rho, \mathcal{E} \vdash \mathcal{F}_{F, P^{\prime}}$.

Therefore, for each $F_{j}$ in $\psi$, there exist $\sigma_{j}^{\prime \prime}, \rho$, and a process $P_{j}$ that follows $F_{j}$ such that $E, \sigma_{j}^{\prime \prime} \oplus \rho, \mathcal{E} \vdash \mathcal{F}_{F_{j}, P_{j}}$. Since the environments $\sigma_{j}^{\prime \prime}$ and $\rho$ have disjoint domains, we can define an environment $\rho^{\prime}=\sigma_{1}^{\prime \prime} \oplus \ldots \oplus \sigma_{m}^{\prime \prime} \oplus \rho$. Then $E, \rho^{\prime}, \mathcal{E} \vdash \mathcal{F}_{F_{1}, P_{1}} \cup \ldots \cup \mathcal{F}_{F_{m}, P_{m}}$.

Let $\theta=\theta\left(P_{1}, \ldots, P_{m}\right)$. Since $E, \rho^{\prime}, \mathcal{E} \vdash \mathcal{F}_{F_{1}, P_{1}} \cup$ $\ldots \cup \mathcal{F}_{F_{m}, P_{m}}$ and $\neg E, \rho^{\prime}, \mathcal{E} \vdash \mathcal{F}\left(P_{1}, \ldots, P_{m}\right)$, we have 
$E, \rho^{\prime}, \mathcal{E} \vdash \theta \phi$. We extend $\rho$ to all $x \in \operatorname{var}(\phi) \backslash \operatorname{var}(\psi)$, in such a way that $E, \rho^{\prime}, \theta(x) \Downarrow \rho(x)$. Moreover, if $x \in \operatorname{var}(\psi)$, then $\rho(x)=\rho(\theta(x))=\rho^{\prime}(\theta(x))$ since $\theta x=x$, so $E, \rho^{\prime}, \theta(x) \Downarrow \rho(x)$. So, for all $x \in \operatorname{var}(\phi)$, $E, \rho^{\prime}, \theta(x) \Downarrow \rho(x)$. Since $E, \rho^{\prime}, \mathcal{E} \vdash \theta \phi$, we have $\rho, \mathcal{E} \vdash \phi$, so $\mathcal{E}$ satisfies the correspondence $\psi \Rightarrow \phi$.

\section{C.2. Injective Correspondences}

$$
\begin{aligned}
& \text { We define formula }\left(\left.\mathcal{F}\right|_{\theta} ^{\mathcal{I}, \mathcal{V}, \mathcal{C}} \phi\right) \text { as follows: } \\
& \text { formula }\left(\mathcal{F} \Leftrightarrow_{\theta}^{\mathcal{I}, \mathcal{V}, \perp} M\right)=\theta M \\
& \operatorname{formula}\left(\mathcal{F} \Leftrightarrow_{\theta}^{\mathcal{I}, \mathcal{V}, \perp} \operatorname{event}\left(e\left(M_{0}, \ldots, M_{m}\right)\right)\right)= \\
& \theta \text { event }\left(e\left(M_{0}, \ldots, M_{m}\right)\right) \\
& \text { formula }\left(\mathcal{F} \Leftrightarrow_{\theta}^{\mathcal{I}, \mathcal{V}, \mathcal{S}} \operatorname{inj-event}\left(e\left(M_{0}, \ldots, M_{m}\right)\right)\right)= \\
& \bigvee\left(\bigwedge_{j=0}^{m} \theta M_{j}=M_{j}^{\prime}\right) \\
& \operatorname{event}\left(e\left(M_{0}^{\prime}, \ldots, M_{m}^{\prime}\right)\right) \in \mathcal{F} \wedge\left(\mathcal{F}, M_{0}^{\prime}, \mathcal{I}, \mathcal{V}\right) \in \mathcal{S} \\
& \text { formula }\left(\mathcal{F} \Leftrightarrow_{\theta}^{\mathcal{I}, \mathcal{V}, \mathcal{C}_{1} \wedge \mathcal{C}_{2}} \phi_{1} \wedge \phi_{2}\right)= \\
& \text { formula }\left(\mathcal{F} \Leftrightarrow_{\theta}^{\mathcal{I}, \mathcal{V}, \mathcal{C}_{1}} \phi_{1}\right) \wedge \text { formula }\left(\mathcal{F} \Leftrightarrow_{\theta}^{\mathcal{I}, \mathcal{V}, \mathcal{C}_{2}} \phi_{2}\right) \\
& \text { formula }\left(\mathcal{F} \Leftrightarrow_{\theta}^{\mathcal{I}, \mathcal{V}, \mathcal{C}_{1} \vee \mathcal{C}_{2}} \phi_{1} \vee \phi_{2}\right)= \\
& \text { formula }\left(\mathcal{F} \Leftrightarrow_{\theta}^{\mathcal{I}, \mathcal{V}, \mathcal{C}_{1}} \phi_{1}\right) \vee \text { formula }\left(\mathcal{F} \Leftrightarrow_{\theta}^{\mathcal{I}, \mathcal{V}, \mathcal{C}_{2}} \phi_{2}\right)
\end{aligned}
$$

where $M_{0}$ is a fresh variable added as first argument of events. The formula formula $\left(\left.\mathcal{F}\right|_{\theta} ^{\mathcal{I}, \mathcal{V}, \mathcal{C}} \phi\right)$ generalizes $\theta \phi$ to the case of injective events.

The next lemma shows that, if $\mathcal{F} \Leftrightarrow_{\theta}^{\mathcal{I}, \mathcal{V}, \mathcal{C}} \phi$, then $\mathcal{F}$ implies formula $\left(\mathcal{F} \models_{\theta}^{\mathcal{I}, \mathcal{V}, \mathcal{C}} \phi\right)$ with overwhelming probability.

Lemma 4 If $\mathcal{F} \Leftrightarrow_{\theta}^{\mathcal{I}, \mathcal{V}, \mathcal{C}} \phi$, then

$$
\operatorname{Pr}\left[C\left[Q_{0}\right] \rightsquigarrow \mathcal{F} \cup\left\{\neg \text { formula }\left(\mathcal{F} \models_{\theta}^{\mathcal{I}, \mathcal{V}, \mathcal{C}} \phi\right)\right\}\right]
$$

is negligible.

Proof The proof is similar to that of Lemma 3, and proceeds by induction on $\phi$. The only new case is the one of injective events.

- Case $\phi=\operatorname{inj}$-event $\left(e\left(M_{0}, \ldots, M_{m}\right)\right), \mathcal{C}=\mathcal{S}$. There are terms $M_{0}^{\prime}, \ldots, M_{m}^{\prime}$ such that event $\left(e\left(M_{0}^{\prime}, \ldots\right.\right.$, $\left.\left.M_{m}^{\prime}\right)\right) \in \mathcal{F}, \mathcal{F} \cup\left\{\bigvee_{j=0}^{m} \theta M_{j} \neq M_{j}^{\prime}\right\}$ yields a contradiction, and $\left(\mathcal{F}, M_{0}^{\gamma}, \mathcal{I}, \mathcal{V}\right) \in \mathcal{S}$. By Proposition 9, $\operatorname{Pr}\left[C\left[Q_{0}\right] \rightsquigarrow \mathcal{F} \cup\left\{\bigvee_{j=0}^{m} \theta M_{j} \neq M_{j}^{\prime}\right\}\right]$ is negligible. Moreover, if $E, \rho, \mathcal{E} \vdash \mathcal{F} \cup\left\{\neg \operatorname{formula}\left(\mathcal{F} \models_{\theta}^{\mathcal{I}, \mathcal{V}, \mathcal{S}} \phi\right)\right\}$, then $E, \rho, \mathcal{E} \vdash \mathcal{F}$ and for all $M_{0}^{\prime}, \ldots, M_{m}^{\prime}$ such that event $\left(e\left(M_{0}^{\prime}, \ldots, M_{m}^{\prime}\right)\right) \in \mathcal{F}$ and $\left(\mathcal{F}, M_{0}^{\prime}, \mathcal{I}, \mathcal{V}\right) \in \mathcal{S}$, in particular for the terms $M_{0}^{\prime}, \ldots, M_{m}^{\prime}$ above, we have $E, \rho, \mathcal{E} \vdash \bigvee_{j=0}^{m} \theta M_{j} \neq M_{j}^{\prime}$, so $E, \rho, \mathcal{E} \vdash$ $\mathcal{F} \cup\left\{\bigvee_{j=0}^{m} \theta M_{j} \neq M_{j}^{\prime}\right\}$. Therefore, $\operatorname{Pr}\left[C\left[Q_{0}\right] \rightsquigarrow\right.$

$$
\begin{aligned}
& \left.\mathcal{F} \cup\left\{\neg \operatorname{formula}\left(\mathcal{F} \models_{\theta}^{\mathcal{I}, \mathcal{V}, \mathcal{S}} \phi\right)\right\}\right] \leq \operatorname{Pr}\left[C\left[Q_{0}\right] \rightsquigarrow \mathcal{F} \cup\right. \\
& \left.\left\{\bigvee_{j=0}^{m} \theta M_{j} \neq M_{j}^{\prime}\right\}\right] . \quad \text { Hence, } \operatorname{Pr}\left[C\left[Q_{0}\right] \rightsquigarrow \mathcal{F} \cup\right. \\
& \left.\left\{\neg \text { formula }\left(\left.\mathcal{F}\right|_{\theta} ^{\mathcal{I}, \mathcal{V}, \mathcal{S}} \phi\right)\right\}\right] \text { is negligible. }
\end{aligned}
$$

Lemma 5 details the meaning of formula $\left(\mathcal{F} \Leftrightarrow_{\theta}^{\mathcal{I}, \mathcal{V}, \mathcal{C}} \phi\right)$. Essentially, this formula implies $\theta \phi$, so, if we store in $\rho(x)$ the value of $\theta(x)$ by $E, \rho^{\prime}, \theta(x) \Downarrow \rho(x)$, we have $\rho, \mathcal{E} \vdash \phi^{\tau}$ $\phi$. Furthermore, for injective events, formula $\left(\left.\mathcal{F}\right|_{\theta} ^{\mathcal{I}, \mathcal{V}, \mathcal{C}} \phi\right)$ guarantees that the quadruples $\left(\mathcal{F}, M_{0}^{\prime}, \mathcal{I}, \mathcal{V}\right)$ are correctly collected in $\mathcal{C}$.

Lemma 5 If $E, \rho^{\prime}, \mathcal{E} \vdash \mathcal{F}$, for all $x \in \operatorname{var}(\phi), E, \rho^{\prime}, \theta(x) \Downarrow$ $\rho(x)$, and $E, \rho^{\prime}, \mathcal{E} \vdash \operatorname{formula}\left(\mathcal{F} \models_{\theta}^{\mathcal{I}, \mathcal{V}, \mathcal{C}} \phi\right)$, then there exists $\phi^{\tau}$ such that $\rho, \mathcal{E} \vdash^{\phi^{\tau}} \phi$ and, if $\tau$ is a nonbottom leaf of $\phi^{\tau}$ and $\mathcal{S}$ the corresponding leaf of $\mathcal{C}$, then $E, \rho^{\prime}, \mathcal{E} \vdash \mathcal{E}(\tau)=\operatorname{event}\left(e\left(M_{0}^{\prime}, \ldots, M_{m}^{\prime}\right)\right)$ for some $\operatorname{event}\left(e\left(M_{0}^{\prime}, \ldots, M_{m}^{\prime}\right)\right) \in \mathcal{F}$ and $\left(\mathcal{F}, M_{0}^{\prime}, \mathcal{I}, \mathcal{V}\right) \in \mathcal{S}$.

Proof The proof proceeds by induction on $\phi$.

- Case $\phi=M$. We have formula $\left(\mathcal{F} \Leftrightarrow_{\theta}^{\mathcal{I}, \mathcal{V}, \mathcal{C}} \phi\right)=\theta M$, so $E, \rho^{\prime}, \mathcal{E} \vdash \theta M$, so $\rho, M \Downarrow$ true, so $\rho, \mathcal{E} \vdash^{\perp} M$. The result holds with $\phi^{\tau}=\perp$.

- Case $\phi=\operatorname{event}\left(e\left(M_{0}, \ldots, M_{m}\right)\right)$. We have

$$
\operatorname{formula}\left(\mathcal{F} \models_{\theta}^{\mathcal{I}, \mathcal{V}, \mathcal{C}} \phi\right)=\theta \operatorname{event}\left(e\left(M_{0}, \ldots, M_{m}\right)\right)
$$

so $E, \rho^{\prime}, \mathcal{E} \vdash \theta$ event $\left(e\left(M_{0}, \ldots, M_{m}\right)\right)$, so $\rho, \mathcal{E} \vdash^{\perp}$ event $\left(e\left(M_{0}, \ldots, M_{m}\right)\right)$. The result holds with $\phi^{\tau}=$ $\perp$.

- Case $\phi=\operatorname{inj-event}\left(e\left(M_{0}, \ldots, M_{m}\right)\right)$. We have

$$
\begin{gathered}
\operatorname{formula}\left(\mathcal{F} \models_{\theta}^{\mathcal{I}, \mathcal{V}, \mathcal{S}} \phi\right)= \\
\bigvee \underset{\operatorname{event}\left(e\left(M_{0}^{\prime}, \ldots, M_{m}^{\prime}\right)\right) \in \mathcal{F} \wedge}{\left(\mathcal{F}, M_{0}^{\prime}, \mathcal{I}, \mathcal{V}\right) \in \mathcal{S}}\left(\bigwedge_{j=0}^{m} \theta M_{j}=M_{j}^{\prime}\right)
\end{gathered}
$$

So there exist $M_{0}^{\prime}, \ldots, M_{m}^{\prime}$ such that event $\left(e\left(M_{0}^{\prime}\right.\right.$, $\left.\left.\ldots, M_{m}^{\prime}\right)\right) \in \mathcal{F},\left(\mathcal{F}, M_{0}^{\prime}, \mathcal{I}, \mathcal{V}\right) \in \mathcal{S}$, and $E, \rho^{\prime}, \mathcal{E} \vdash$ $\bigwedge_{j=0}^{m} \theta M_{j}=M_{j}^{\prime}$. We have $E, \rho^{\prime}, \mathcal{E} \vdash \mathcal{F}$, so $E, \rho^{\prime}, \mathcal{E} \vdash \operatorname{event}\left(e\left(M_{0}^{\prime}, \ldots, M_{m}^{\prime}\right)\right)$, so $E, \rho^{\prime}, \mathcal{E} \vdash$ $\theta$ event $\left(e\left(M_{0}, \ldots, M_{m}\right)\right)$, so there exists $\tau$ such that $\mathcal{E}(\tau)=\operatorname{event}\left(e\left(a_{0}, \ldots, a_{m}\right)\right)$ with for all $j \leq m$, $E, \rho^{\prime}, \theta M_{j} \Downarrow a_{j}$, so for all $j \leq m, E, \rho, M_{j} \Downarrow a_{j}$, so $\rho, \mathcal{E} \vdash^{\tau}$ event $\left.\left(M_{0}, \ldots, M_{m}\right)\right)$. Moreover, $E, \rho^{\prime}, \mathcal{E} \vdash$ $\mathcal{E}(\tau)=\operatorname{event}\left(e\left(a_{0}, \ldots, a_{m}\right)\right)=\theta \operatorname{event}\left(e\left(M_{0}, \ldots\right.\right.$, $\left.\left.M_{m}\right)\right)=\operatorname{event}\left(e\left(M_{0}^{\prime}, \ldots, M_{m}^{\prime}\right)\right)$. As already noticed, we have event $\left(e\left(M_{0}^{\prime}, \ldots, M_{m}^{\prime}\right)\right) \in \mathcal{F}$ and $\left(\mathcal{F}, M_{0}^{\prime}, \mathcal{I}\right.$, $\mathcal{V}) \in \mathcal{S}$, so the result holds with $\phi^{\tau}=\tau$. 
- Case $\phi=\phi_{1} \wedge \phi_{2}$. We have

$$
E, \rho^{\prime}, \mathcal{E} \vdash \operatorname{formula}\left(\mathcal{F} \models_{\theta}^{\mathcal{I}, \mathcal{V}, \mathcal{C}_{1} \wedge \mathcal{C}_{2}} \phi_{1} \wedge \phi_{2}\right)
$$

So

$$
\begin{aligned}
& E, \rho^{\prime}, \mathcal{E} \vdash \operatorname{formula}\left(\mathcal{F} \models_{\theta}^{\mathcal{I}, \mathcal{V}, \mathcal{C}_{1}} \phi_{1}\right) \text { and } \\
& E, \rho^{\prime}, \mathcal{E} \vdash \operatorname{formula}\left(\mathcal{F} \models_{\theta}^{\mathcal{I}, \mathcal{V}, \mathcal{C}_{2}} \phi_{2}\right)
\end{aligned}
$$

The induction hypothesis yields $\phi_{1}^{\tau}$ and $\phi_{2}^{\tau}$, and the result holds with $\phi^{\tau}=\phi_{1}^{\tau} \wedge \phi_{2}^{\tau}$.

- Case $\phi=\phi_{1} \vee \phi_{2}$. We have

$$
E, \rho^{\prime}, \mathcal{E} \vdash \operatorname{formula}\left(\left.\mathcal{F}\right|_{\theta} ^{\mathcal{I}, \mathcal{V}, \mathcal{C}_{1} \vee \mathcal{C}_{2}} \phi_{1} \vee \phi_{2}\right)
$$

SO

$$
\begin{aligned}
& E, \rho^{\prime}, \mathcal{E} \vdash \operatorname{formula}\left(\mathcal{F} \mapsto_{\theta}^{\mathcal{I}, \mathcal{V}, \mathcal{C}_{1}} \phi_{1}\right) \text { or } \\
& E, \rho^{\prime}, \mathcal{E} \vdash \operatorname{formula}\left(\left.\mathcal{F}\right|_{\theta} ^{\mathcal{I}, \mathcal{V}, \mathcal{C}_{2}} \phi_{2}\right)
\end{aligned}
$$

In the first case, the induction hypothesis yields $\phi_{1}^{\tau}$, and the result holds with $\phi^{\tau}=\phi_{1}^{\tau} \vee \phi_{2}^{\perp}$, where $\phi_{2}^{\perp}$ is the formula $\phi_{2}$ in which all terms and events have been replaced with $\perp$. The second case follows by symmetry.

The next lemma shows that, for events $e$ used as injective events, two distinct executions of event $e$ have distinct replication indices. This is a consequence of the requirement that two occurrences of the same event $e$ be in different branches of find or if in $Q_{0}$.

When the term $M$ contains no array accesses, we define $\sigma(M)$ by $E, \sigma, M \Downarrow \sigma(M)$ for any environment $E$, since the evaluation of $M$ does not depend on $E$.

Lemma 6 Assume that the event e is used as injective event in the correspondence $\psi \Rightarrow \phi$. Let $C$ be an evaluation context acceptable for $Q_{0}$ with public variables $V$ that does not contain events used by $\psi \Rightarrow \phi$. If the trace initConfig $\left(C\left[Q_{0}\right]\right) \stackrel{\mathcal{E}}{\rightarrow} p, \mathcal{T} \mathbb{C}$ contains two distinct reductions

$$
\begin{gathered}
E,\left(\sigma, \text { event } e\left(M_{0}, \ldots, M_{m}\right) ; P\right), \mathcal{Q}, \mathcal{C} \\
\stackrel{e\left(a_{0}, \ldots, a_{m}\right)}{\longrightarrow}{ }_{1, E v} E,(\sigma, P), \mathcal{Q}, \mathcal{C} \\
\text { and } E^{\prime},\left(\sigma^{\prime}, \text { event } e\left(M_{0}^{\prime}, \ldots, M_{m}^{\prime}\right) ; P^{\prime}\right), \mathcal{Q}^{\prime}, \mathcal{C}^{\prime} \\
\stackrel{e\left(a_{0}^{\prime}, \ldots, a_{m}^{\prime}\right)}{\longrightarrow}{ }_{1, E v} E^{\prime},\left(\sigma^{\prime}, P^{\prime}\right), \mathcal{Q}^{\prime}, \mathcal{C}^{\prime}
\end{gathered}
$$

then $a_{0} \neq a_{0}^{\prime}$.

Proof Let us fix the event symbol $e$. We define Events (initConfig $\left(C\left[Q_{0}\right]\right) \stackrel{\mathcal{E}}{\rightarrow}_{p, \mathcal{T}} \mathbb{C}$ ) as the multiset that contains $a_{0}$ for each reduction $E$, $\left(\sigma\right.$, event $e\left(M_{0}, \ldots\right.$,
$\left.\left.M_{m}\right) ; P\right), \mathcal{Q}, \mathcal{C} \stackrel{e\left(a_{0}, \ldots, a_{m}\right)}{\longrightarrow}{ }_{1, E v} E,(\sigma, P), \mathcal{Q}, \mathcal{C}$ in the trace initConfig $\left(C\left[Q_{0}\right]\right) \stackrel{\mathcal{E}}{\rightarrow} p, \mathcal{T} \mathbb{C}$. Multisets $S$ are represented by functions that map each element $x$ of $S$ to the number of occurrences of $x$ in $S$. When $S_{1}$ and $S_{2}$ are multisets, multiset union $S_{1} \uplus S_{2}$ is defined by $\left(S_{1} \uplus S_{2}\right)(x)=$ $S_{1}(x)+S_{2}(x)$, and the multiset $\max \left(S_{1}, S_{2}\right)$ is defined by $\max \left(S_{1}, S_{2}\right)(x)=\max \left(S_{1}(x), S_{2}(x)\right)$. We define the multisets $\operatorname{Events}(\sigma, P)$ and $\operatorname{Events}(\sigma, Q)$ by

$$
\begin{aligned}
& \operatorname{Events}(\sigma, 0)=\emptyset \\
& \operatorname{Events}\left(\sigma, Q_{1} \mid Q_{2}\right)=\operatorname{Events}\left(\sigma, Q_{1}\right) \uplus \operatorname{Events}\left(\sigma, Q_{2}\right) \\
& \operatorname{Events}\left(\sigma, !^{i \leq n} Q\right)=\biguplus_{a \in\left[1, I_{\eta}(n)\right]} \operatorname{Events}(\sigma[i \mapsto a], Q)
\end{aligned}
$$

$\operatorname{Events}(\sigma$, newChannel $c ; Q)=\operatorname{Events}(\sigma, Q)$

Events $\left(\sigma, c\left[M_{1}, \ldots, M_{l}\right]\left(x_{1} \widetilde{[i]}: T_{1}, \ldots, x_{k} \widetilde{[i]}: T_{k}\right) ; P\right)=$ Events $(\sigma, P)$

$\operatorname{Events}\left(\sigma, \overline{c\left[M_{1}, \ldots, M_{l}\right]}\left\langle N_{1}, \ldots, N_{k}\right\rangle ; Q\right)=$

$\operatorname{Events}(\sigma, Q)$

$\operatorname{Events}(\sigma$, new $x \widetilde{[i]}: T ; P)=\operatorname{Events}(\sigma, P)$

$\operatorname{Events}(\sigma$, let $x \widetilde{i i}]: T=M$ in $P)=\operatorname{Events}(\sigma, P)$

$\operatorname{Events}\left(\sigma\right.$, event $\left.e^{\prime}\left(M_{0}, \ldots, M_{m}\right) ; P\right)=$

$\operatorname{Events}(\sigma, P)$ if $e^{\prime} \neq e$

$\operatorname{Events}\left(\sigma\right.$, event $\left.e\left(M_{0}, \ldots, M_{m}\right) ; P\right)=$

$\left\{\sigma\left(M_{0}\right)\right\} \uplus \operatorname{Events}(\sigma, P)$

Events $\left(\sigma\right.$, find $\left(\bigoplus_{j=1}^{m} \widetilde{u_{j}} \widetilde{[i]} \leq \widetilde{n_{j}}\right.$ suchthat

$$
\begin{gathered}
\left.\left.\operatorname{defined}\left(M_{j 1}, \ldots, M_{j l_{j}}\right) \wedge M_{j} \text { then } P_{j}\right) \text { else } P\right)= \\
\max \left(\max _{j=1}^{m} \operatorname{Events}\left(\sigma, P_{j}\right), \operatorname{Events}(\sigma, P)\right)
\end{gathered}
$$

We define the multiset Events $(E,(\sigma, P), \mathcal{Q}, \mathcal{C})=$ Events $(\sigma, P) \uplus \biguplus_{\left(\sigma^{\prime}, Q^{\prime}\right) \in \mathcal{Q}}$ Events $\left(\sigma^{\prime}, Q^{\prime}\right)$. This multiset contains all bitstrings $a_{0}$ for events $e(\ldots)$ that may be executed in a trace that begins with $E,(\sigma, P), \mathcal{Q}, \mathcal{C}$.

The multiset Events(initConfig $\left.\left(C\left[Q_{0}\right]\right)\right)$ contains no duplicates, since two occurrences of the same event $e$ must be in different branches of find or if in $Q_{0}$ and $C$ does not contain event $e$. Moreover, for the empty trace $\epsilon$, Events $(\epsilon)=\emptyset$, so Events $(\epsilon) \uplus$ Events(initConfig $\left.\left(C\left[Q_{0}\right]\right)\right)$ contains no duplicates.

We show that, if initConfig $\left(C\left[Q_{0}\right]\right) \stackrel{\mathcal{E}}{\rightarrow} p, \mathcal{T} \mathbb{C} \stackrel{[e]}{\longrightarrow}_{p^{\prime}, t^{\prime}}$ $\mathbb{C}^{\prime}$, then Events (initConfig $\left.\left(C\left[Q_{0}\right]\right) \stackrel{\mathcal{E}}{\rightarrow}_{p, \mathcal{T}} \stackrel{[e]}{\longrightarrow}_{p^{\prime}, t^{\prime}} \quad \mathbb{C}^{\prime}\right) \uplus$

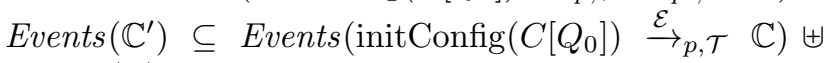
Events $(\mathbb{C})$.

Thus, if initConfig $\left(C\left[Q_{0}\right]\right) \stackrel{\mathcal{E}}{\rightarrow}_{p, \mathcal{T}} \mathbb{C}$, then

$$
\begin{aligned}
& \text { Events (initConfig } \left.\left(C\left[Q_{0}\right]\right) \stackrel{\mathcal{E}}{\rightarrow}_{p, \mathcal{T}} \mathbb{C}\right) \uplus \operatorname{Events}(\mathbb{C}) \\
& \subseteq \text { Events }(\epsilon) \uplus \text { Events }\left(\operatorname{initConfig}\left(C\left[Q_{0}\right]\right)\right)
\end{aligned}
$$


These multisets contain no duplicates, so in particular,

$$
\text { Events }\left(\operatorname{initConfig}\left(C\left[Q_{0}\right]\right) \stackrel{\mathcal{E}}{\rightarrow}_{p, \mathcal{T}} \mathbb{C}\right)
$$

contains no duplicates. This property implies the desired result.

Proof of Proposition 2 By hypothesis, if $P_{1}$ follows $F_{1}, \ldots$, and $P_{m}$ follows $F_{m}$, then there exists a substitution $\theta$ equal to the identity on the variables of $\psi$ and such that $\left.\mathcal{F}\right|_{\theta} ^{\mathcal{I}, \mathcal{V}, \mathcal{C}} \phi$ where $\mathcal{F}=\mathcal{F}_{F_{1}, P_{1}} \cup \ldots \cup \mathcal{F}_{F_{m}, P_{m}}$, $\mathcal{I}=\left\{j \mapsto I_{P_{j}} \mid F_{j}\right.$ is an injective event $\}$, and $\mathcal{V}=$ $\operatorname{var}\left(I_{P_{1}}\right) \cup \ldots \cup \operatorname{var}\left(I_{P_{m}}\right) \cup \operatorname{var}(\psi)$. We let $\theta\left(P_{1}, \ldots P_{m}\right)$ be such a substitution and we define $\mathcal{F}\left(P_{1}, \ldots, P_{m}\right)=$ $\mathcal{F} \cup\left\{\neg\right.$ formula $\left.\left(\mathcal{F} \Leftrightarrow_{\theta}^{\mathcal{I}, \mathcal{V}, \mathcal{C}} \phi\right)\right\}$ where $\theta=\theta\left(P_{1}, \ldots, P_{m}\right)$.

Let $C$ be an evaluation context acceptable for $Q_{0}$ with public variables $V$ that does not contain events used by $\psi \Rightarrow \phi$. Next, we show that if initConfig $\left(C\left[Q_{0}\right]\right) \stackrel{\mathcal{E}}{\rightarrow}_{p, \mathcal{T}}$ $E,(\sigma, P), \mathcal{Q}, \mathcal{C}$ and $\mathcal{E} \forall \psi \Rightarrow \phi$, then

- there exist $P_{1}$ that follows $F_{1}, \ldots, P_{m}$ that follows $F_{m}$, and $\rho^{\prime}$ such that $E, \rho^{\prime}, \mathcal{E} \vdash \mathcal{F}\left(P_{1}, \ldots, P_{m}\right)$,

- or there exist a non-bottom leaf $\mathcal{S}$ of $\mathcal{C},\left(\mathcal{F}, M_{0}, \mathcal{I}, \mathcal{V}\right)$ and $\left(\mathcal{F}^{\prime}, M_{0}^{\prime}, \mathcal{I}^{\prime}, \mathcal{V}^{\prime}\right)$ in $\mathcal{S}$, and $\rho^{\prime}$ such that $E, \rho^{\prime}, \mathcal{E} \vdash$ $\mathcal{F} \cup \mathcal{F}^{\prime} \cup\left\{\bigvee_{j \in \operatorname{Dom}(\mathcal{I})} \mathcal{I}(j) \neq \theta^{\prime \prime} \mathcal{I}^{\prime}(j)\right\} \cup\left\{\theta^{\prime \prime} M_{0}^{\prime}=\right.$ $\left.M_{0}\right\}$, where the substitution $\theta^{\prime \prime}$ is a renaming of the variables in $\mathcal{V}^{\prime}$ to distinct fresh variables.

Therefore,

$$
\begin{aligned}
& \operatorname{Pr}\left[C\left[Q_{0}\right]: \neg(\psi \Rightarrow \phi)\right] \\
& \leq \operatorname{Pr}\left[\begin{array}{l}
\exists(E, \sigma, P, \mathcal{Q}, \mathcal{C}, \mathcal{E}), \\
\operatorname{initConfig}\left(C\left[Q_{0}\right]\right) \stackrel{\mathcal{E}}{\rightarrow} E,(\sigma, P), \mathcal{Q}, \mathcal{C} \wedge \\
\mathcal{E} \nvdash \psi \Rightarrow \phi
\end{array}\right] \\
& \leq \sum_{P_{1}, \ldots, P_{m} \text { that }} \operatorname{Pr}\left[\begin{array}{l}
\exists\left(E, \sigma, P, \mathcal{Q}, \mathcal{C}, \rho^{\prime}, \mathcal{E}\right), \\
\operatorname{initConfig}\left(C\left[Q_{0}\right]\right) \stackrel{\mathcal{E}}{\rightarrow} E,(\sigma, P), \mathcal{Q}, \mathcal{C} \\
\wedge E, \rho^{\prime}, \mathcal{E} \vdash \mathcal{F}\left(P_{1}, \ldots, P_{m}\right)
\end{array}\right] \\
& \text { follow } F_{1}, \ldots, F_{m} \text { respectively } \\
& +\sum_{\mathcal{S} \text { leaf of } \mathcal{C}, \mathcal{S} \neq \perp,} \operatorname{Pr}\left[\begin{array}{c}
\exists\left(E, \sigma, P, \mathcal{Q}, \mathcal{C}, \rho^{\prime}, \mathcal{E}\right), \\
\operatorname{initConfig}\left(C\left[Q_{0}\right]\right) \stackrel{\mathcal{E}}{\rightarrow} E,(\sigma, P), \mathcal{Q}, \mathcal{C} \\
\wedge E, \rho^{\prime}, \mathcal{E} \vdash \mathcal{F} \cup \mathcal{F}^{\prime} \cup\left\{M_{0}=\theta^{\prime \prime} M_{0}^{\prime}\right\} \\
\cup\left\{\bigvee_{j \in \operatorname{Dom}(\mathcal{I})} \mathcal{I}(j) \neq \theta^{\prime \prime} \mathcal{I}^{\prime}(j)\right\}
\end{array}\right] \\
& \left(\mathcal{F}^{\prime}, M_{0}^{\prime}, \mathcal{I}^{\prime}, \mathcal{V}^{\prime}\right) \in \mathcal{S} \\
& \leq \sum_{P_{1}, \ldots, P_{m} \text { that follow } F_{1}, \ldots, F_{m} \text { respectively }} \operatorname{Pr}\left[C\left[Q_{0}\right] \rightsquigarrow \mathcal{F}\left(P_{1}, \ldots, P_{m}\right)\right] \\
& +\sum_{\mathcal{S} \text { leaf of } \mathcal{C}, \mathcal{S} \neq \perp,} \operatorname{Pr}\left[\begin{array}{rl}
C\left[Q_{0}\right] & \rightsquigarrow \mathcal{F} \cup \mathcal{F}^{\prime} \cup\left\{M_{0}=\theta^{\prime \prime} M_{0}^{\prime}\right\} \\
\cup\left\{\bigvee_{j \in \operatorname{Dom}(\mathcal{I})} \mathcal{I}(j) \neq \theta^{\prime \prime} \mathcal{I}^{\prime}(j)\right\}
\end{array}\right] \\
& \left(\mathcal{F}, M_{0}, \mathcal{I}, \mathcal{V}\right) \in \mathcal{S},\left(\mathcal{F}^{\prime}, M_{0}^{\prime}, \mathcal{I}^{\prime}, \mathcal{V}^{\prime}\right) \in \mathcal{S}
\end{aligned}
$$

By Lemma 4 , since $\mathcal{F} \Leftrightarrow_{\theta}^{\mathcal{I}, \mathcal{V}, \mathcal{C}} \phi$, the probability $\operatorname{Pr}\left[C\left[Q_{0}\right]\right.$ $\left.\rightsquigarrow \mathcal{F} \cup\left\{\neg \operatorname{formula}\left(\left.\mathcal{F}\right|_{\theta} ^{\mathcal{I}, \mathcal{V}, \mathcal{C}} \phi\right)\right\}\right]$ is negligible, that is, $\operatorname{Pr}\left[C\left[Q_{0}\right] \rightsquigarrow \mathcal{F}\left(P_{1}, \ldots, P_{m}\right)\right]$ is negligible. Since $\vdash \mathcal{C}$, for all non-bottom leaves $\mathcal{S}$ of $\mathcal{C}$, for all $\left(\mathcal{F}, M_{0}, \mathcal{I}, \mathcal{V}\right)$, $\left(\mathcal{F}^{\prime}, M_{0}^{\prime}, \mathcal{I}^{\prime}, \mathcal{V}^{\prime}\right)$ in $\mathcal{S}, \mathcal{F} \cup \theta^{\prime \prime} \mathcal{F}^{\prime} \cup\left\{\bigvee_{j \in \operatorname{Dom}(\mathcal{I})} \mathcal{I}(j) \neq\right.$ $\left.\theta^{\prime \prime} \mathcal{I}^{\prime}(j), M_{0}=\theta^{\prime \prime} M_{0}^{\prime}\right\}$ yields a contradiction. By Proposition 9, $\operatorname{Pr}\left[C\left[Q_{0}\right] \rightsquigarrow \mathcal{F} \cup \mathcal{F}^{\prime} \cup\left\{M_{0}=\theta^{\prime \prime} M_{0}^{\prime}\right\} \cup\right.$ $\left.\left\{\bigvee_{j \in \operatorname{Dom}(\mathcal{I})} \mathcal{I}(j) \neq \theta^{\prime \prime} \mathcal{I}^{\prime}(j)\right\}\right]$ is negligible. Hence the sum is negligible, so $Q_{0}$ satisfies the correspondence $\psi \Rightarrow$ $\phi$ with public variables $V$.

Assume that

- $\operatorname{initConfig}\left(C\left[Q_{0}\right]\right) \stackrel{\mathcal{E}}{\rightarrow}_{p, \mathcal{T}} E,(\sigma, P), \mathcal{Q}, \mathcal{C}$,

- for every $P_{1}$ that follows $F_{1}, \ldots$, for every $P_{m}$ that follows $F_{m}$, for every $\rho^{\prime}$, we have $\neg E, \rho^{\prime}, \mathcal{E} \vdash \mathcal{F}\left(P_{1}\right.$, $\left.\ldots, P_{m}\right)$,

- and for every non-bottom leaf $\mathcal{S}$ of $\mathcal{C}$, for every $\left(\mathcal{F}, M_{0}, \mathcal{I}, \mathcal{V}\right)$ and $\left(\mathcal{F}^{\prime}, M_{0}^{\prime}, \mathcal{I}^{\prime}, \mathcal{V}^{\prime}\right)$ in $\mathcal{S}$, for every $\rho^{\prime}$, we have $\neg E, \rho^{\prime}, \mathcal{E} \vdash \mathcal{F} \cup \mathcal{F}^{\prime} \cup\left\{\bigvee_{j \in \operatorname{Dom}(\mathcal{I})} \mathcal{I}(j) \neq\right.$ $\left.\theta^{\prime \prime} \mathcal{I}^{\prime}(j)\right\} \cup\left\{M_{0}=\theta^{\prime \prime} M_{0}^{\prime}\right\}$, where the substitution $\theta^{\prime \prime}$ is a renaming of the variables in $\mathcal{V}^{\prime}$ to distinct fresh variables.

We show that $\mathcal{E} \vdash \psi \Rightarrow \phi$.

Assume that $\rho, \mathcal{E} \vdash \psi^{\tau} \psi$, where $\rho$ is defined on $\operatorname{var}(\psi)$, $\psi=F_{1} \wedge \ldots \wedge F_{m}, \psi^{\tau}=\tau_{1} \wedge \ldots \wedge \tau_{m}$, and for all $j \leq m$, $\tau_{j}$ is either a step or $\perp$. For each event $F_{j}=\operatorname{event}\left(e_{j}\left(M_{j 0}\right.\right.$, $\left.\left.\ldots, M_{j m_{j}}\right)\right)$ or $F_{j}=\operatorname{inj-event}\left(e_{j}\left(M_{j 0}, \ldots, M_{j m_{j}}\right)\right)$ in $\psi$, we have $\rho, \mathcal{E} \vdash^{\tau_{j}} \operatorname{event}\left(e_{j}\left(M_{j 0}, \ldots, M_{j m_{j}}\right)\right)$, so $\rho, M_{j k} \Downarrow a_{j k}$ for all $k \leq m_{j}$ and $e_{j}\left(a_{j 0}, \ldots, a_{j m_{j}}\right) \in \mathcal{E}$. Moreover, if $F_{j}=\operatorname{inj-event}\left(e_{j}\left(M_{j 0}, \ldots, M_{j m_{j}}\right)\right)$, then $e_{j}\left(a_{j 0}, \ldots, a_{j m_{j}}\right)=\mathcal{E}\left(\tau_{j}\right)$. Since the only transition that produces a label $e_{j}\left(a_{j 0}, \ldots, a_{j m_{j}}\right)$ is (Event), the trace $\operatorname{initConfig}\left(Q_{0}\right) \stackrel{\mathcal{E}}{\rightarrow} p, \mathcal{T} E,(\sigma, P), \mathcal{Q}, \mathcal{C}$ contains a transition of the form $E_{j},\left(\sigma_{j}\right.$, event $\left.e_{j}\left(M_{j 0}^{\prime}, \ldots, M_{j m_{j}}^{\prime}\right) ; P_{j}\right)$, $\mathcal{Q}_{j}, \mathcal{C}_{j} \stackrel{e_{j}\left(a_{j 0}, \ldots, a_{j m_{j}}\right)}{\longrightarrow} 1, E v \quad E_{j},\left(\sigma_{j}, P_{j}\right), \mathcal{Q}_{j}, \mathcal{C}_{j} \quad$ with $E_{j}, \sigma_{j}, M_{j k}^{\prime} \Downarrow a_{j k}$ for all $k \leq m_{j}$. By Proposition 5, event $e_{j}\left(M_{j 0}^{\prime}, \ldots, M_{j m_{j}}^{\prime}\right) ; P_{j}$ is a subprocess of $C\left[Q_{0}\right]$ or of $\overline{\text { start }}\langle\rangle ; 0$. Since $C$ does not contain events used by $\psi \Rightarrow$ $\phi$, event $e_{j}\left(M_{j 0}^{\prime}, \ldots, M_{j m_{j}}^{\prime}\right) ; P_{j}$ is a subprocess of $Q_{0}$, so $P_{j}$ follows $F_{j}$. By Proposition $7, E_{j}, \sigma_{j}, \mathcal{E}_{j} \vdash \mathcal{F}_{P_{j}}$, where $\mathcal{E}_{j}$ is the prefix of $\mathcal{E}$ until and including the considered occurrence of the event $e_{j}\left(a_{j 0}, \ldots, a_{j m_{j}}\right)$. By Proposition 6, $E$ is an extension of $E_{j}$, so $E, \sigma_{j}, \mathcal{E} \vdash \mathcal{F}_{P_{j}}$. Let $\theta_{j}^{\prime}$ be the substitution that renames replication indices at $P_{j}$ to fresh replication indices, such that $\mathcal{F}_{F_{j}, P_{j}}=\theta_{j}^{\prime} \mathcal{F}_{P_{j}} \cup\left\{\theta_{j}^{\prime} M_{j k}^{\prime}=\right.$ $\left.M_{j k} \mid k \leq m_{j}\right\}$ and $I_{P_{j}}=\theta_{j}^{\prime} M_{j 0}^{\prime}$ since the tuple of replication indices at $P_{j}$ is added as first argument $M_{j 0}^{\prime}$ of events in $Q_{0}$. Let $\sigma_{j}^{\prime}$ be such that $\sigma_{j}=\sigma_{j}^{\prime} \theta_{j}^{\prime}$. Then $E, \sigma_{j}^{\prime}, \mathcal{E} \vdash$ $\theta_{j} \mathcal{F}_{P_{j}}$. For all $k \leq m_{j}$, since $E_{j}, \sigma_{j}, M_{j k}^{\prime} \Downarrow a_{j k}$, we have $E, \sigma_{j}^{\prime}, \theta_{j}^{\prime} M_{j k}^{\prime} \Downarrow a_{j k}$. We have $\rho, M_{j k} \Downarrow a_{j k}$. Hence 
$E, \sigma_{j}^{\prime} \oplus \rho, \mathcal{E} \vdash \theta_{j}^{\prime} M_{j k}^{\prime}=M_{j k}$, where $\sigma_{j}^{\prime} \oplus \rho$ denotes the function that maps $x$ to $\sigma_{j}^{\prime}(x)$ when $x \in \operatorname{Dom}\left(\sigma_{j}^{\prime}\right)$ and $i$ to $\rho(i)$ when $i \in \operatorname{Dom}(\rho)$. So $E, \sigma_{j}^{\prime} \oplus \rho, \mathcal{E} \vdash \mathcal{F}_{F_{j}, P_{j}}$.

Therefore, for each $j \leq m$, there exists a process $P_{j}$ that follows $F_{j}$ such that

- for all $j \leq m$, there is a reduction

$$
\begin{gathered}
E_{j},\left(\sigma_{j}, \text { event } e_{j}\left(M_{j 0}^{\prime}, \ldots, M_{j m_{j}}^{\prime}\right) ; P_{j}\right), \mathcal{Q}_{j}, \mathcal{C}_{j} \\
\stackrel{e_{j}\left(a_{j 0}, \ldots, a_{j m_{j}}\right)}{\longrightarrow} \\
\stackrel{1, E v}{ } E_{j},\left(\sigma_{j}, P_{j}\right), \mathcal{Q}_{j}, \mathcal{C}_{j}
\end{gathered}
$$

in the trace initConfig $\left(C\left[Q_{0}\right]\right) \stackrel{\mathcal{E}}{\rightarrow}_{p, \mathcal{T}} E,(\sigma, P), \mathcal{Q}, \mathcal{C}$, and if $F_{j}=\operatorname{inj-event}\left(e_{j}(\ldots)\right)$, then $\tau_{j} \neq \perp$ and $\mathcal{E}\left(\tau_{j}\right)=e_{j}\left(a_{j 0}, \ldots, a_{j m_{j}}\right)$

- letting $\rho^{\prime}=\sigma_{1}^{\prime} \oplus \ldots \oplus \sigma_{m}^{\prime} \oplus \rho$, we have $\operatorname{Dom}\left(\rho^{\prime}\right)=$ $\operatorname{var}\left(I_{P_{1}}\right) \cup \ldots \cup \operatorname{var}\left(I_{P_{m}}\right) \cup \operatorname{var}(\psi), E, \rho^{\prime}, \mathcal{E} \vdash \mathcal{F}_{F_{1}, P_{1}} \cup$ $\ldots \cup \mathcal{F}_{F_{m}, P_{m}}$ and for all $j \leq m, \rho^{\prime}\left(I_{P_{j}}\right)=\sigma_{j}^{\prime}\left(I_{P_{j}}\right)=$ $\sigma_{j}\left(M_{j 0}^{\prime}\right)=a_{j 0}$.

Let $\theta=\theta\left(P_{1}, \ldots, P_{m}\right)$. Let $\mathcal{F}=\mathcal{F}_{F_{1}, P_{1}} \cup \ldots \cup \mathcal{F}_{F_{m}, P_{m}}$, $\mathcal{I}=\left\{j \mapsto I_{P_{j}} \mid F_{j}\right.$ is an injective event $\}$, and $\mathcal{V}=$ $\operatorname{var}\left(I_{P_{1}}\right) \cup \ldots \cup \operatorname{var}\left(I_{P_{m}}\right) \cup \operatorname{var}(\psi)$. Since $E, \rho^{\prime}, \mathcal{E} \vdash$ $\mathcal{F}_{F_{1}, P_{1}} \cup \ldots \cup \mathcal{F}_{F_{m}, P_{m}}$ and $\neg E, \rho^{\prime}, \mathcal{E} \vdash \mathcal{F}\left(P_{1}, \ldots, P_{m}\right)$, we have $E, \rho^{\prime}, \mathcal{E} \vdash$ formula $\left(\mathcal{F} \models_{\theta}^{\mathcal{I}, \mathcal{V}, \mathcal{C}} \phi\right)$. We extend $\rho$ to all $x \in \operatorname{var}(\phi) \backslash \operatorname{var}(\psi)$ in such a way that $E, \rho^{\prime}, \theta(x) \Downarrow \rho(x)$. Then, for all $x \in \operatorname{var}(\phi) \cup \operatorname{var}(\psi), E, \rho^{\prime}, \theta(x) \Downarrow \rho(x)$. By Lemma 5, there exists $\phi^{\tau}$ such that $\rho, \mathcal{E} \vdash^{\phi^{\tau}} \phi$ and, if $\tau$ is a non-bottom leaf of $\phi^{\tau}$ and $\mathcal{S}$ the corresponding leaf of $\mathcal{C}$, then $E, \rho^{\prime}, \mathcal{E} \vdash \mathcal{E}(\tau)=\operatorname{event}\left(e\left(M_{0}^{\prime \prime}, \ldots, M_{m}^{\prime \prime}\right)\right)$ for some event $\left(e\left(M_{0}^{\prime \prime}, \ldots, M_{m}^{\prime \prime}\right)\right) \in \mathcal{F}$ and $\left(\mathcal{F}, M_{0}^{\prime \prime}, \mathcal{I}, \mathcal{V}\right) \in \mathcal{S}$.

We define $\mathbb{F}$ as the function that maps $\psi^{\tau}$ to $\phi^{\tau}$ build as above. It suffices to show that $\mathbb{F}$ is component-wise injective. Let $f$ be a projection of $\mathbb{F}$ to a leaf of $\phi$, and $\mathcal{S}$ the corresponding leaf of $\mathcal{C}$. Assume that $f\left(\psi_{1}^{\tau}\right)=f\left(\psi_{2}^{\tau}\right)=$ $\tau \neq \perp$. Let us show that $\psi_{1}^{\tau}=\psi_{2}^{\tau}$.

Assume that $\psi_{1}^{\tau}=\tau_{11} \wedge \ldots \wedge \tau_{1 m}$ and $\psi_{2}^{\tau}=\tau_{21} \wedge \ldots \wedge$ $\tau_{2 m}$. By construction of $\mathbb{F}$, we have

- for all $j \leq m$, there is a reduction

$$
\begin{gathered}
E_{1 j},\left(\sigma_{1 j}, \text { event } e_{j}\left(M_{1 j 0}^{\prime}, \ldots, M_{1 j m_{j}}^{\prime}\right) ; P_{1 j}\right), \mathcal{Q}_{1 j}, \mathcal{C}_{1 j} \\
\stackrel{e_{j}\left(a_{1 j 0}, \ldots, a_{1 j m_{j}}\right)}{\longrightarrow}{ }_{1, E v} E_{1 j},\left(\sigma_{1 j}, P_{1 j}\right), \mathcal{Q}_{1 j}, \mathcal{C}_{1 j}
\end{gathered}
$$

in the trace initConfig $\left(C\left[Q_{0}\right]\right) \stackrel{\mathcal{E}}{\rightarrow}_{p, \mathcal{T}} E,(\sigma, P), \mathcal{Q}, \mathcal{C}$, and if $F_{j}=\operatorname{inj-event}\left(e_{j}(\ldots)\right)$, then $\tau_{1 j} \neq \perp$ and $\mathcal{E}\left(\tau_{1 j}\right)=e_{j}\left(a_{1 j 0}, \ldots, a_{1 j m_{j}}\right) ; E, \rho_{1}^{\prime}, \mathcal{E} \vdash$ $\mathcal{F}_{1}$, for all $j \leq m, \rho_{1}^{\prime}\left(I_{P_{1 j}}\right)=a_{1 j 0}, \mathcal{I}_{1}=$ $\left\{j \mapsto I_{P_{1 j}} \mid F_{j}\right.$ is an injective event $\}, E, \rho_{1}^{\prime}, \mathcal{E} \vdash$ $\mathcal{E}(\tau)=\operatorname{event}\left(e\left(M_{1}^{\prime \prime}, \ldots\right)\right),\left(\mathcal{F}_{1}, M_{1}^{\prime \prime}, \mathcal{I}_{1}, \mathcal{V}_{1}\right) \in \mathcal{S}$, and $\operatorname{Dom}\left(\rho_{1}^{\prime}\right)=\mathcal{V}_{1}$;
- for all $j \leq m$, there is a reduction

$$
\begin{gathered}
E_{2 j},\left(\sigma_{2 j}, \text { event } e_{j}\left(M_{2 j 0}^{\prime}, \ldots, M_{2 j m_{j}}^{\prime}\right) ; P_{2 j}\right), \mathcal{Q}_{2 j}, \mathcal{C}_{2 j} \\
\stackrel{e_{j}\left(a_{2 j 0}, \ldots, a_{2 j m_{j}}\right)}{\longrightarrow} 1, E v \\
\stackrel{E_{2 j}}{\longrightarrow},\left(\sigma_{2 j}, P_{2 j}\right), \mathcal{Q}_{2 j}, \mathcal{C}_{2 j}
\end{gathered}
$$

in the trace initConfig $\left(C\left[Q_{0}\right]\right) \stackrel{\mathcal{E}}{\rightarrow}_{p, \mathcal{T}} E,(\sigma, P), \mathcal{Q}, \mathcal{C}$, and if $F_{j}=\operatorname{inj-event}\left(e_{j}(\ldots)\right)$, then $\tau_{2 j} \neq \perp$ and $\mathcal{E}\left(\tau_{2 j}\right)=e_{j}\left(a_{2 j 0}, \ldots, a_{2 j m_{j}}\right) ; E, \rho_{2}^{\prime}, \mathcal{E} \vdash$ $\mathcal{F}_{2}$, for all $j \leq m, \rho_{2}^{\prime}\left(I_{P_{2 j}}\right)=a_{2 j 0}, \mathcal{I}_{2}=$ $\left\{j \mapsto I_{P_{2 j}} \mid F_{j}\right.$ is an injective event $\}, E, \rho_{2}^{\prime}, \mathcal{E} \vdash$ $\mathcal{E}(\tau)=\operatorname{event}\left(e\left(M_{2}^{\prime \prime}, \ldots\right)\right),\left(\mathcal{F}_{2}, M_{2}^{\prime \prime}, \mathcal{I}_{2}, \mathcal{V}_{2}\right) \in \mathcal{S}$, and $\operatorname{Dom}\left(\rho_{2}^{\prime}\right)=\mathcal{V}_{2}$.

Let $\theta^{\prime \prime}$ be a renaming that maps variables of $\mathcal{V}_{2}$ to distinct fresh variables. Let $\rho^{\prime}$ be defined by $\rho^{\prime}(x)=\rho_{1}^{\prime}(x)$ if $x \in$ $\mathcal{V}_{1}$ and $\rho^{\prime}(x)=\rho_{2}^{\prime}\left(\theta^{\prime \prime-1}(x)\right)$ if $x \in \theta^{\prime \prime}\left(\mathcal{V}_{2}\right)$.

Then $E, \rho^{\prime}, \mathcal{E} \vdash \mathcal{F}_{1}, E, \rho^{\prime}, \mathcal{E} \vdash \theta^{\prime \prime} \mathcal{F}_{2}, E, \rho^{\prime}, \mathcal{E} \vdash$ $\operatorname{event}\left(e\left(M_{1}^{\prime \prime}, \ldots\right)\right)=\mathcal{E}(\tau)=\theta^{\prime \prime} \operatorname{event}\left(e\left(M_{2}^{\prime \prime}, \ldots\right)\right)$, so $E, \rho^{\prime}, \mathcal{E} \vdash M_{1}^{\prime \prime}=\theta^{\prime \prime} M_{2}^{\prime \prime}$. Hence by hypothesis, $E, \rho^{\prime}, \mathcal{E} \vdash$ $\bigwedge_{j \in \operatorname{Dom}\left(\mathcal{I}_{1}\right)} \mathcal{I}_{1}(j)=\theta^{\prime \prime} \mathcal{I}_{2}(j)$, so for all $j \in \operatorname{Dom}\left(\mathcal{I}_{1}\right)$, $\rho^{\prime}\left(\mathcal{I}_{1}(j)\right)=\rho^{\prime}\left(\theta^{\prime \prime} \mathcal{I}_{2}(j)\right)$, that is, $\rho_{1}^{\prime}\left(I_{P_{1 j}}\right)=\rho_{2}^{\prime}\left(I_{P_{2 j}}\right)$, so $a_{1 j 0}=a_{2 j 0}$. By Lemma 6 , for all $j \in \operatorname{Dom}\left(\mathcal{I}_{1}\right)$, that is, for all $j$ such that $F_{j}$ is an injective event, there is a single reduction in the trace with a label of the form $e_{j}\left(a_{1 j 0}, \ldots\right)$, so $\tau_{1 j}=\tau_{2 j}$. Furthermore, for all $j$ such that $F_{j}$ is a noninjective event, $\tau_{1 j}=\tau_{2 j}=\perp$. So $\psi_{1}^{\tau}=\psi_{2}^{\tau}$.

Hence $\mathbb{F}$ is component-wise injective, so $\mathcal{E} \vdash \psi \Rightarrow \phi$. This concludes the proof.

\section{C.3. Authenticated Key Exchange}

Proof of Proposition 4 We first show that $Q_{0}$ is a secure mutual authentication protocol. The first condition of Definition 7 holds by hypothesis, and it implies the first condition of Definition 6. The last two conditions of Definition 6 come from (5) and (6), as in Proposition 3.

Next, we show the second condition of Definition 7. We define a process $Q_{1}$ obtained from $Q_{0}$ by adding

- event $\operatorname{part}_{A}\left(Y, \operatorname{sid}^{\prime}\left(x_{1}, \ldots, x_{r-1}\right)\right)$; event $\operatorname{full}_{A}\left(Y, k_{A}, \operatorname{sid}\left(x_{1}, \ldots, x_{r}\right)\right)$ just before $\overline{c_{A r}\left[i_{A}\right]}\left\langle x_{r}, \operatorname{accept}_{A}(Y)\right\rangle$,

- event $\operatorname{part}_{B}\left(X, \operatorname{sid}^{\prime}\left(y_{1}, \ldots, y_{r-1}\right)\right)$ just before $\overline{c_{B r-1}\left[i_{B}\right]}\left\langle y_{r-1}\right\rangle$, and

- event $\operatorname{full}_{B}\left(X, k_{B}, \operatorname{sid}\left(y_{1}, \ldots, y_{r}\right)\right)$ just before $\overline{c_{B r+1}\left[i_{B}\right]}\left\langle\operatorname{accept}_{B}(X)\right\rangle$.

Let $Q_{2}$ be the process obtained from $Q_{0}^{\prime}$ by deleting events. We define

$Q_{k_{A}^{\prime}}=!^{i \leq n^{\prime}} c\left(u_{S}:[1, n]\right) ;$ if defined $\left(k_{A}^{\prime}\left[u_{S}\right]\right)$ then
$\quad \bar{c}\left\langle k_{A}^{\prime}\left[u_{S}\right]\right\rangle$ 


$$
\begin{gathered}
Q_{k_{A}^{\prime}}^{\prime}=!^{i \leq n^{\prime}} c\left(u_{S}:[1, n]\right) ; \text { if } \operatorname{defined}\left(k_{A}^{\prime}\left[u_{S}\right]\right) \text { then } \\
\text { find } u^{\prime} \leq n^{\prime} \text { suchthat defined }\left(y\left[u^{\prime}\right], u_{S}\left[u^{\prime}\right]\right) \wedge \\
u_{S}\left[u^{\prime}\right]=u_{S} \text { then } \bar{c}\left\langle y\left[u^{\prime}\right]\right\rangle \text { else new } y: T ; \bar{c}\langle y\rangle
\end{gathered}
$$

Since $Q_{0}^{\prime}$ preserves the secrecy of $k_{A}^{\prime}$, we have $Q_{2} \mid Q_{k_{A}^{\prime}} \approx$ $Q_{2} \mid Q_{k_{A}^{\prime}}^{\prime}$

Below, we define a process $Q_{S T}$ that simulates the test queries of $Q_{T}$ by calling the process $Q_{0}^{\prime} \mid Q_{k_{A}^{\prime}}$ and the test queries of $Q_{T}^{\prime}$ by calling $Q_{0}^{\prime} \mid Q_{k_{A}^{\prime}}^{\prime}$, so that

$$
\begin{aligned}
& Q_{1} \mid Q_{T} \approx \text { newChannel } \tilde{c}^{\prime} ;\left(\left(Q_{0}^{\prime} \mid Q_{k_{A}^{\prime}}\right)\left\{\widetilde{c^{\prime}} / \widetilde{c}\right\} \mid Q_{S T}\right) \\
& Q_{1} \mid Q_{T}^{\prime} \approx \text { newChannel } \widetilde{c^{\prime}} ;\left(\left(Q_{0}^{\prime} \mid Q_{k_{A}^{\prime}}^{\prime}\right)\left\{\widetilde{c^{\prime}} / \widetilde{c}\right\} \mid Q_{S T}\right)
\end{aligned}
$$

where $\widetilde{c}=\left(c_{A 0}, \ldots, c_{A r}, c_{A K}, c_{B 1}, \ldots, c_{B r}, c_{B K}, c\right)$ and $\widetilde{c^{\prime}}$ consists of fresh names such that $\widetilde{c^{\prime}}=\left(c_{A 0}^{\prime}, \ldots, c_{A r}^{\prime}\right.$, $\left.c_{A K}^{\prime}, c_{B 1}^{\prime}, \ldots, c_{B r}^{\prime}, c_{B K}^{\prime}, c^{\prime}\right)$. By deleting events, we have

$$
\begin{aligned}
& Q_{0} \mid Q_{T} \approx \text { newChannel } \tilde{c}^{\prime} ;\left(\left(Q_{2} \mid Q_{k_{A}^{\prime}}\right)\left\{\widetilde{c^{\prime}} / \widetilde{c}\right\} \mid Q_{S T}\right) \\
& Q_{0} \mid Q_{T}^{\prime} \approx \text { newChannel } \widetilde{c^{\prime}} ;\left(\left(Q_{2} \mid Q_{k_{A}^{\prime}}^{\prime}\right)\left\{\widetilde{c^{\prime}} / \widetilde{c}\right\} \mid Q_{S T}\right)
\end{aligned}
$$

Since $Q_{2}\left|Q_{k_{A}^{\prime}} \approx Q_{2}\right| Q_{k_{A}^{\prime}}^{\prime}$, we have by renaming $\left(Q_{2} \mid Q_{k_{A}^{\prime}}\right)\left\{\tilde{c^{\prime}} / \widetilde{c}\right\} \approx\left(Q_{2} \mid Q_{k_{A}^{\prime}}^{\prime}\right)\left\{\tilde{c^{\prime}} / \widetilde{c}\right\}$. Moreover, $Q_{S T}$ does not use the variables of $Q_{2}, Q_{k_{A}^{\prime}}, Q_{k_{A}^{\prime}}^{\prime}$, so by Lemma 2, Property 2, newChannel $\widetilde{c^{\prime}} ;\left(\left(Q_{2} \mid Q_{k_{A}^{\prime}}\right)\left\{\widetilde{c^{\prime}} / \widetilde{c}\right\} \mid\right.$ $\left.Q_{S T}\right) \approx$ newChannel $\widetilde{c^{\prime}} ;\left(\left(Q_{2} \mid Q_{k_{A}^{\prime}}^{\prime}\right)\left\{\widetilde{c^{\prime}} / \widetilde{c}\right\} \mid Q_{S T}\right)$.

Then $Q_{0} \mid Q_{T} \approx$ newChannel $\widetilde{c^{\prime}} ;\left(\left(Q_{2} \mid Q_{k_{A}^{\prime}}\right)\left\{\widetilde{c^{\prime}} / \widetilde{c}\right\} \mid\right.$ $\left.Q_{S T}\right) \approx$ newChannel $\widetilde{c^{\prime}} ;\left(\left(Q_{2} \mid Q_{k_{A}^{\prime}}^{\prime}\right)\left\{\widetilde{c^{\prime}} / \widetilde{c}\right\} \mid Q_{S T}\right) \approx$ $Q_{0} \mid Q_{T}^{\prime}$, so by transitivity $Q_{0}\left|Q_{T} \approx Q_{0}\right| Q_{T}^{\prime}$, which proves the desired result.

We now define the process $Q_{S T}$; we explain this definition below. We define $\widetilde{x}[M]$ as an abbreviation for $x_{1}[M], \ldots, x_{r}[M]$ and we define $\widetilde{x^{\prime}}[M], \widetilde{y}[M]$, and $\widetilde{y^{\prime}}[M]$ similarly. We let $Q_{S T}=Q_{S T A}\left|Q_{S T B}\right| Q_{R A} \mid Q_{R B}$ where

$$
\begin{aligned}
& Q_{S T A}=!^{i \leq n_{T}} \text { test }_{A}[i]\left(u_{A}\right) ; \\
& \text { if defined }\left(x_{r+1}^{\prime}\left[u_{A}\right]\right) \wedge x_{r+1}^{\prime}\left[u_{A}\right] \neq \text { reject then } \\
& \text { if } x_{r+1}^{\prime}\left[u_{A}\right] \neq \operatorname{accept}_{A}(B) \text { then } \\
& \quad \overline{c_{A K}^{\prime}\left[u_{A}\right]}\langle\rangle ; c_{A K}^{\prime}\left[u_{A}\right](k) ; \overline{\text { test }_{A}[i]}\langle k\rangle \\
& \text { else } \\
& \text { find } u \leq n_{T} \text { suchthat defined }\left(u_{A}[u], r_{A}[u]\right) \wedge \\
& \qquad u_{A}[u]=u_{A} \text { then } \overline{\operatorname{test}_{A}[i]}\left\langle r_{A}[u]\right\rangle \text { else } \\
& \text { find } u \leq n_{T} \text { suchthat } \operatorname{defined}\left(u_{B}[u], r_{B}[u],\right. \\
& \left.\quad \widetilde{x^{\prime}}\left[u_{A}\right], \widetilde{y^{\prime}}\left[u_{B}[u]\right]\right) \wedge \operatorname{sid}\left(\widetilde{x^{\prime}}\left[u_{A}\right]\right)=\operatorname{sid}\left(\widetilde{y^{\prime}}\left[u_{B}[u]\right]\right) \\
& \quad \text { then } \overline{\operatorname{test}_{A}[i]}\left\langle r_{B}[u]\right\rangle \operatorname{else} \\
& \overline{c^{\prime}[i]}\left\langle u_{A}\right\rangle ; c^{\prime}[i]\left(r_{A}\right) ; \overline{\operatorname{test}_{A}[i]}\left\langle r_{A}\right\rangle
\end{aligned}
$$

$$
\begin{aligned}
& Q_{S T B}=!^{i \leq n_{T}} \text { test }_{B}[i]\left(u_{B}\right) \text {; } \\
& \text { if defined }\left(y_{r+1}^{\prime}\left[u_{B}\right]\right) \wedge y_{r+1}^{\prime}\left[u_{B}\right] \neq \text { reject then } \\
& \text { if } y_{r+1}^{\prime}\left[u_{B}\right] \neq \operatorname{accept}_{B}(A) \text { then } \\
& \overline{c_{B K}^{\prime}\left[u_{B}\right]}\langle\rangle ; c_{B K}^{\prime}\left[u_{B}\right](k) ; \overline{\text { test }_{B}[i]}\langle k\rangle \\
& \text { else } \\
& \text { find } u \leq n_{T} \text { suchthat defined }\left(u_{B}[u], r_{B}[u]\right) \wedge \\
& u_{B}[u]=u_{B} \text { then } \overline{\text { test }_{B}[i]}\left\langle r_{B}[u]\right\rangle \text { else } \\
& \text { find } u \leq n_{T} \text { suchthat defined }\left(u_{A}[u], r_{A}[u]\right. \text {, } \\
& \left.\widetilde{x^{\prime}}\left[u_{A}[u]\right], \widetilde{y^{\prime}}\left[u_{B}\right]\right) \wedge \operatorname{sid}\left(\widetilde{x^{\prime}}\left[u_{A}[u]\right]\right)=\operatorname{sid}\left(\widetilde{y^{\prime}}\left[u_{B}\right]\right) \\
& \text { then } \overline{\text { test }_{B}[i]}\left\langle r_{A}[u]\right\rangle \text { else } \\
& \text { find } u_{A}^{\prime} \leq n \text { suchthat defined }\left(\widetilde{x^{\prime}}\left[u_{A}^{\prime}\right], \widetilde{y^{\prime}}\left[u_{B}\right]\right. \text {, } \\
& \left.x_{r+1}^{\prime}\left[u_{A}^{\prime}\right]\right) \wedge \operatorname{sid}\left(\widetilde{x^{\prime}}\left[u_{A}^{\prime}\right]\right)=\operatorname{sid}\left(\widetilde{y^{\prime}}\left[u_{B}\right]\right) \wedge \\
& x_{r+1}^{\prime}\left[u_{A}^{\prime}\right]=\operatorname{accept}_{A}(B) \text { then } \\
& \overline{c^{\prime}\left[i+n_{T}\right]}\left\langle u_{A}^{\prime}\right\rangle ; c^{\prime}\left[i+n_{T}\right]\left(r_{B}\right) ; \overline{\text { test }_{B}[i]}\left\langle r_{B}\right\rangle \\
& Q_{R A}=!^{i \leq n} c_{A 0}[i]\left(Y^{\prime}\right) ; \overline{c_{A 0}^{\prime}[i]}\left\langle Y^{\prime}\right\rangle \text {; } \\
& c_{A 1}^{\prime}[i]\left(x_{1}^{\prime}\right) ; \overline{c_{A 1}[i]}\left\langle x_{1}^{\prime}\right\rangle ; c_{A 2}[i]\left(x_{2}^{\prime}\right) ; \overline{c_{A 2}^{\prime}[i]}\left\langle x_{2}^{\prime}\right\rangle ; \\
& \ldots ; c_{A r}^{\prime}[i]\left(x_{r}^{\prime}, x_{r+1}^{\prime}\right) ; \overline{c_{A r}[i]}\left\langle x_{r}^{\prime}, x_{r+1}^{\prime}\right\rangle \\
& Q_{R B}=!^{i \leq n} c_{B 1}[i]\left(y_{1}^{\prime}\right) ; \overline{c_{B 1}^{\prime}[i]}\left\langle y_{1}^{\prime}\right\rangle \text {; } \\
& c_{B 2}^{\prime}[i]\left(y_{2}^{\prime}\right) ; \overline{c_{B 2}[i]}\left\langle y_{2}^{\prime}\right\rangle ; \ldots ; c_{B r}[i]\left(y_{r}^{\prime}\right) ; \overline{c_{B r}^{\prime}[i]}\left\langle y_{r}^{\prime}\right\rangle ; \\
& c_{B r+1}^{\prime}[i]\left(y_{r+1}^{\prime}\right) ; \overline{c_{B r+1}[i]}\left\langle y_{r+1}^{\prime}\right\rangle
\end{aligned}
$$

where $I_{\eta}\left(n^{\prime}\right)=2 \times I_{\eta}\left(n_{T}\right)$ ( $n^{\prime}$ is the number of queries allowed in $Q_{k_{A}^{\prime}}$ and $Q_{k_{A}^{\prime}}^{\prime}$ ) and all variables in these processes are fresh. (The variables $Y^{\prime}, x_{j}^{\prime}, y_{j}^{\prime}$ play the same role as $Y, x_{j}, y_{j}$ in $Q_{0}^{\prime}$; they have been renamed to avoid confusion with the variables of $Q_{0}^{\prime}$.) The processes $Q_{R A}$ and $Q_{R B}$ relay the requests from channels $c_{A j}$ and $c_{B j}$ to channels $c_{A j}^{\prime}$ and $c_{B j}^{\prime}$. These relay processes are useful in order to store the messages in $x_{1}^{\prime}, \ldots, x_{r+1}^{\prime}, y_{1}^{\prime}, \ldots, y_{r+1}^{\prime}$, to have access to them without reading the variables of $Q_{0}^{\prime}$.

The processes $Q_{S T A}$ and $Q_{S T B}$ simulate the test queries. They first check that the queried copy of $Q_{A}$ or $Q_{B}$ has accepted (first test of $Q_{S T A}$ and $Q_{S T B}$ ). Then, if the queried session is not between $A$ and $B$, they call $Q_{0}^{\prime}$ to return the session key. Otherwise, they call $Q_{k_{A}^{\prime}}$ to return the key of sessions between $A$ and $B$, or $Q_{k_{A}^{\prime}}^{\prime}$ to return a fresh random number for each session between $A$ and $B$. Before calling $Q_{k_{A}^{\prime}}$ or $Q_{k_{A}^{\prime}}^{\prime}$, they first check if the same test query (or a test query to the partner) has already been called, and if it has, they return the previously returned value. (These checks are not strictly necessary, because $Q_{k_{A}^{\prime}}^{\prime}$ already checks if the same query has already been called. However, they slightly simplify the proof by making the structure of $Q_{S T A}$ and $Q_{S T B}$ closer to the structure $Q_{T A}^{\prime}$ and $Q_{T B}^{\prime}$.) After these checks, $Q_{S T A}$ calls $Q_{k_{A}^{\prime}}$ or $Q_{k_{A}^{\prime}}^{\prime}$ directly (last line of $Q_{S T A}$ ) while $Q_{S T B}$ first uses 
a find to find the copy of $Q_{A}$, partner of the considered session and calls $Q_{k_{A}^{\prime}}$ or $Q_{k_{A}^{\prime}}^{\prime}$ for that partner (last find of $\left.Q_{S T B}\right)$.

To show an equivalence $L \approx R$, we show that, after excluding a set of traces of negligible probability, each trace of $L$ can be simulated by a trace of $R$ of the same probability, and conversely.

For the equivalence

$$
Q_{1} \mid Q_{T} \approx \text { newChannel } \widetilde{c^{\prime}} ;\left(\left(Q_{0}^{\prime} \mid Q_{k_{A}^{\prime}}\right)\left\{\widetilde{c^{\prime}} / \widetilde{c}\right\} \mid Q_{S T}\right)
$$

the proof is done by considering only the traces in which the correspondences (5)-(7) hold. The other traces have negligible probability since the correspondences (5)-(7) are satisfied by $Q_{0}^{\prime}$ with public variables $\left\{k_{A}^{\prime}\right\}$, so by Lemma 1 , they are also satisfied by newChannel $\widetilde{c^{\prime}}$; $\left(\left(Q_{0}^{\prime} \mid Q_{k_{A}^{\prime}}\right)\left\{\widetilde{c^{\prime}} / \widetilde{c}\right\} \mid Q_{S T}\right)$ and newChannel $\widetilde{c}^{\prime} ;\left(\left(Q_{0}^{\prime} \mid\right.\right.$ $\left.\left.Q_{k_{A}^{\prime}}^{\prime}\right)\left\{\widetilde{c^{\prime}} / \widetilde{c}\right\} \mid Q_{S T}\right)$. We establish the correspondence between traces by induction on the length of the trace:

- When $Q_{1} \mid Q_{T}$ receives a message on channel $c_{A j}\left[i_{A}\right]$ with $j<r-1, Q_{1}$ stores the received message in $x_{j}\left[i_{A}\right]$, answers by returning the next message of the protocol $x_{j+1}\left[i_{A}\right]$ on $c_{A j+1}\left[i_{A}\right]$. Correspondingly, in $\left(\left(Q_{0}^{\prime} \mid Q_{k_{A}^{\prime}}\right)\left\{\widetilde{c^{\prime}} / \widetilde{c}\right\} \mid Q_{S T}\right), Q_{R A}$ stores the received message in $x_{j}^{\prime}\left[i_{A}\right]$ (or $Y^{\prime}\left[i_{A}\right]$ if $j=0$ ), forwards it on $c_{A j}^{\prime}\left[i_{A}\right] ; Q_{0}^{\prime}\left\{\widetilde{c^{\prime}} / \widetilde{c}\right\}$ answers to it like $Q_{1}$ except that the next message $x_{j+1}\left[i_{A}\right]$ is sent on $c_{A j+1}^{\prime}\left[i_{A}\right]$; $Q_{R A}$ then stores this message in $x_{j+1}^{\prime}\left[i_{A}\right]$ and forwards it on $c_{A j+1}\left[i_{A}\right]$. When $j=r-1$, the situation is similar, except that the returned message is a pair $x_{r}\left[i_{A}\right]$, accept ${ }_{A}\left(Y\left[i_{A}\right]\right)$ or $x_{r}\left[i_{A}\right]$, reject, stored by $Q_{R A}$ in $x_{r}^{\prime}\left[i_{A}\right], x_{r+1}^{\prime}\left[i_{A}\right]$. When $j=r-1$ and the protocol accepts, both sides define $k_{A}\left[i_{A}\right]$, execute event $\operatorname{full}_{A}\left(Y\left[i_{A}\right], k_{A}\left[i_{A}\right], \operatorname{sid}\left(\widetilde{x}\left[i_{A}\right]\right)\right)$, and send $x_{r}\left[i_{A}\right]$, accept ${ }_{A}\left(Y\left[i_{A}\right]\right)$, so in the right-hand side $x_{r+1}^{\prime}\left[i_{A}\right]=\operatorname{accept}_{A}\left(Y\left[i_{A}\right]\right)$. When furthermore $Y\left[i_{A}\right]=B, Q_{0}^{\prime}$ additionally defines $k_{A}^{\prime}\left[i_{A}\right]=k_{A}\left[i_{A}\right]$. When $j=r-1$ and the protocol rejects, $k_{A}\left[i_{A}\right]$ is not defined and both sides send $x_{r}\left[i_{A}\right]$, reject, so in the right-hand side $x_{r+1}^{\prime}\left[i_{A}\right]=$ reject.

So, $k_{A}\left[i_{A}\right]$ is defined in the left-hand side if and only if $x_{r+1}^{\prime}\left[i_{A}\right]$ is defined and different from reject in the right-hand side, and in this case, $Y\left[i_{A}\right], \widetilde{x}\left[i_{A}\right]$, and $k_{A}\left[i_{A}\right]$ have the same value in both sides of the equivalence and, in the right-hand side, $Y^{\prime}\left[i_{A}\right]=Y\left[i_{A}\right]$, $\widetilde{x^{\prime}}\left[i_{A}\right]=\widetilde{x}\left[i_{A}\right], k_{A}^{\prime}\left[i_{A}\right]=k_{A}\left[i_{A}\right]$ if $Y\left[i_{A}\right]=B$, $k_{A}^{\prime}\left[i_{A}\right]$ is not defined if $Y\left[i_{A}\right] \neq B$, and $x_{r+1}^{\prime}\left[i_{A}\right]=$ $\operatorname{accept}_{A}\left(Y\left[i_{A}\right]\right)$.

- Similarly, the messages on $c_{B j}\left[i_{B}\right]$ are answered in the same way by both sides of the equivalence, thanks to the forwarding by $Q_{R B}$ in the right-hand side.
So, $k_{B}\left[i_{B}\right]$ is defined in the left-hand side if and only if $y_{r+1}^{\prime}\left[i_{B}\right]$ is defined and different from reject in the right-hand side, and in this case, $X\left[i_{B}\right], \widetilde{y}\left[i_{B}\right]$, and $k_{B}\left[i_{B}\right]$ have the same value in both sides of the equivalence and, in the right-hand side, $X^{\prime}\left[i_{B}\right]=X\left[i_{B}\right]$, $\widetilde{y^{\prime}}\left[i_{B}\right]=\widetilde{y}\left[i_{B}\right]$, and $y_{r+1}^{\prime}\left[i_{B}\right]=\operatorname{accept}_{B}\left(X\left[i_{B}\right]\right)$.

- When $Q_{1} \mid Q_{T}$ receives a message test $_{A}[i]\left(u_{A}\right), Q_{T A}$ returns $k_{A}\left[u_{A}\right]$ if it is defined. Correspondingly, in the right-hand side, $Q_{S T A}$ first tests if $x_{r+1}^{\prime}\left[u_{A}\right]$ is defined and different from reject, which is equivalent to $k_{A}\left[u_{A}\right]$ defined, as mentioned above.

If $x_{r+1}^{\prime}\left[u_{A}\right] \neq \operatorname{accept}_{A}(B)$, then $Y\left[u_{A}\right] \neq B$. In this case, $Q_{S T A}$ sends an empty message on $c_{A K}^{\prime}\left[u_{A}\right]$. $Q_{0}^{\prime}$ receives it, and sends $k_{A}\left[u_{A}\right]$ on $c_{A K}^{\prime}\left[u_{A}\right]$. $Q_{S T A}$ then receives this message, stores it in $k$, and sends $k=k_{A}\left[u_{A}\right]$ on test $_{A}[i]$, as in the left-hand side.

Otherwise, $x_{r+1}^{\prime}\left[u_{A}\right]=\operatorname{accept}_{A}(B)$ and $Y\left[u_{A}\right]=B$. Then $Q_{S T A}$ checks if the same test query has been asked before (test query number $u$ such that $u_{A}[u]$ and $r_{A}[u]$ are defined, and $u_{A}[u]=u_{A}$ ). Below, we show that, when $r_{A}[i]$ is defined, $k_{A}\left[u_{A}[i]\right]$ and $Y\left[u_{A}[i]\right]$ are defined, $r_{A}[i]=k_{A}\left[u_{A}[i]\right]$, and $Y\left[u_{A}[i]\right]=B$. So $r_{A}[u]=k_{A}\left[u_{A}[u]\right]=k_{A}\left[u_{A}\right]$, and $k_{A}\left[u_{A}\right]$ is sent on test $_{A}[i]$, as in the left-hand side.

Next, $Q_{S T A}$ checks if a test query has been asked to the partner of $Q_{A}^{u_{A}}$ (test query number $u$ such that $u_{B}[u], r_{B}[u], \widetilde{x^{\prime}}\left[u_{A}\right]$, and $\widetilde{y^{\prime}}\left[u_{B}[u]\right]$ are defined and $\left.\operatorname{sid}\left(\widetilde{x^{\prime}}\left[u_{A}\right]\right)=\operatorname{sid}\left(\widetilde{y^{\prime}}\left[u_{B}[u]\right]\right)\right)$. Below, we show that, when $r_{B}[i]$ is defined, $k_{B}\left[u_{B}[i]\right]$ and $X\left[u_{B}[i]\right]$ are defined, $r_{B}[i]=k_{B}\left[u_{B}[i]\right]$, and $X\left[u_{B}[i]\right]=$ A. So $r_{B}[u]=k_{B}\left[u_{B}[u]\right]$. Since $k_{B}\left[u_{B}[u]\right]$ and $x_{r}^{\prime}\left[u_{A}\right]$ are defined, the events $\operatorname{full}_{B}\left(X\left[u_{B}[u]\right]\right.$, $\left.k_{B}\left[u_{B}[u]\right], \operatorname{sid}\left(\widetilde{y}\left[u_{B}[u]\right]\right)\right)$ and $\operatorname{full}_{A}\left(Y\left[u_{A}\right], k_{A}\left[u_{A}\right]\right.$, $\left.\operatorname{sid}\left(\widetilde{x}\left[u_{A}\right]\right)\right)$ have been executed. Since $\operatorname{sid}\left(\widetilde{x}\left[u_{A}\right]\right)=$ $\operatorname{sid}\left(\widetilde{x^{\prime}}\left[u_{A}\right]\right)=\operatorname{sid}\left(\widetilde{y^{\prime}}\left[u_{B}[u]\right]\right)=\operatorname{sid}\left(\widetilde{y}\left[u_{B}[u]\right]\right)$, $X\left[u_{B}[u]\right]=A$, and $Y\left[u_{A}\right]=B$, these events are $\operatorname{full}_{B}\left(A, k_{B}\left[u_{B}[u]\right], \operatorname{sid}\left(\widetilde{x}\left[u_{A}\right]\right)\right)$ and $f_{u l l}(B$, $\left.k_{A}\left[u_{A}\right], \operatorname{sid}\left(\widetilde{x}\left[u_{A}\right]\right)\right)$. So by the correspondence (7), $k_{B}\left[u_{B}[u]\right]=k_{A}\left[u_{A}\right]$, hence $r_{B}[u]=k_{B}\left[u_{B}[u]\right]=$ $k_{A}\left[u_{A}\right]$ is sent on test $_{A}[i]$, as in the left-hand side.

Finally, if both finds fail, then $Q_{S T A}$ sends $u_{A}$ on $c^{\prime}[i] . \quad Q_{k_{A}^{\prime}}\left\{\widetilde{c^{\prime}} / \widetilde{c}\right\}$ receives this message and replies by sending $k_{A}^{\prime}\left[u_{A}\right]=k_{A}\left[u_{A}\right]$ on $c^{\prime}[i]$. $Q_{S T A}$ stores the reply in $r_{A}$, so $r_{A}=k_{A}\left[u_{A}\right]$, and sends $k_{A}\left[u_{A}\right]$ on test $_{A}[i]$, as in the left-hand side. Moreover, we have $Y\left[u_{A}\right]=B$ so, spelling out all array indices, $r_{A}[i]=k_{A}\left[u_{A}[i]\right]$ and $Y\left[u_{A}[i]\right]=B$.

- When $Q_{1} \mid Q_{T}$ receives a message test $_{B}[i]\left(u_{B}\right)$, the situation is almost symmetric of the previous case. We just detail the case in which $y_{r+1}^{\prime}\left[u_{B}\right]=$ 
accept $_{B}(A)$ and the first two finds of $Q_{S T B}$ fail. We have $X\left[u_{B}\right]=A$. Then the event $\operatorname{full}_{B}\left(A, k_{B}\left[u_{B}\right]\right.$, $\left.\operatorname{sid}\left(\widetilde{y}\left[u_{B}\right]\right)\right)$ has been executed. By the correspondence (6), the event $\operatorname{full}_{A}\left(B, k_{B}\left[u_{B}\right], \operatorname{sid}\left(\widetilde{y}\left[u_{B}\right]\right)\right)$ has been executed. So there exists $u_{A}^{\prime \prime}$ such that $Y^{\prime}\left[u_{A}^{\prime \prime}\right]=$ $Y\left[u_{A}^{\prime \prime}\right]=B, k_{A}\left[u_{A}^{\prime \prime}\right]=k_{B}\left[u_{B}\right], \operatorname{sid}\left(\widetilde{x^{\prime}}\left[u_{A}^{\prime \prime}\right]\right)=$ $\operatorname{sid}\left(\widetilde{x}\left[u_{A}^{\prime \prime}\right]\right)=\operatorname{sid}\left(\widetilde{y}\left[u_{B}\right]\right), x_{r+1}^{\prime}\left[u_{A}^{\prime \prime}\right]=\operatorname{accept}_{A}(B)$. So the last find of $Q_{S T B}$ succeeds for some value of $u_{A}^{\prime}$. Moreover, since $x_{r}^{\prime}\left[u_{A}^{\prime}\right]$ is defined, the event $\operatorname{full}_{A}\left(Y\left[u_{A}^{\prime}\right], k_{A}\left[u_{A}^{\prime}\right], \operatorname{sid}\left(\widetilde{x}\left[u_{A}^{\prime}\right]\right)\right)$ has been executed. Since $x_{r+1}^{\prime}\left[u_{A}^{\prime}\right]=\operatorname{accept}_{A}(B), Y\left[u_{A}^{\prime}\right]=$ $Y^{\prime}\left[u_{A}^{\prime}\right]=B$ and $\operatorname{sid}\left(\widetilde{x}\left[u_{A}^{\prime}\right]\right)=\operatorname{sid}\left(\widetilde{x^{\prime}}\left[u_{A}^{\prime}\right]\right)=$ $\operatorname{sid}\left(\widetilde{y^{\prime}}\left[u_{B}\right]\right)=\operatorname{sid}\left(\widetilde{y}\left[u_{B}\right]\right)$, this event is $\operatorname{full}_{A}(B$, $\left.k_{A}\left[u_{A}^{\prime}\right], \operatorname{sid}\left(\widetilde{y}\left[u_{B}\right]\right)\right) . \quad$ By the correspondence (7), $k_{A}\left[u_{A}^{\prime}\right]=k_{B}\left[u_{B}\right]$. The process $Q_{S T B}$ sends $u_{A}^{\prime}$ on channel $c^{\prime}\left[i+n_{T}\right]$. This message is received by $Q_{k_{A}^{\prime}}$. Moreover, $k_{A}^{\prime}\left[u_{A}^{\prime}\right]$ is defined and $k_{A}^{\prime}\left[u_{A}^{\prime}\right]=k_{A}\left[u_{A}^{\prime}\right]$, since $x_{r}^{\prime}\left[u_{A}^{\prime}\right]$ is defined and $Y\left[u_{A}^{\prime}\right]=B$. Then $Q_{k_{A}^{\prime}}$ replies by sending $k_{A}^{\prime}\left[u_{A}^{\prime}\right]$ on channel $c^{\prime}\left[i+n_{T}\right]$. Then $r_{B}=k_{A}^{\prime}\left[u_{A}^{\prime}\right]=k_{A}\left[u_{A}^{\prime}\right]=k_{B}\left[u_{B}\right]$, and $k_{B}\left[u_{B}\right]$ is sent on test $_{B}[i]$, as in the left-hand side.

For the equivalence

$$
Q_{1} \mid Q_{T}^{\prime} \approx \text { newChannel } \widetilde{c^{\prime}} ;\left(\left(Q_{0}^{\prime} \mid Q_{k_{A}^{\prime}}^{\prime}\right)\left\{\widetilde{c^{\prime}} / \widetilde{c}\right\} \mid Q_{S T}\right)
$$

we exclude not only the traces that do not satisfy the correspondences (5)-(7), but also the traces in which $k_{A}^{\prime}[u]=$ $k_{A}^{\prime}\left[u^{\prime}\right]$ for some $u \neq u^{\prime}$. These traces have negligible probability, because otherwise that would contradict the secrecy of $k_{A}^{\prime}$ : the adversary could distinguish $Q_{0}^{\prime} \mid Q_{k_{A}^{\prime}}$ from $Q_{0}^{\prime} \mid Q_{k_{A}^{\prime}}^{\prime}$ with non-negligible probability, by detecting the former when he obtains the same answer to queries $\overline{c^{\prime}[i]}\langle u\rangle$ and $\overline{c^{\prime}[i]}\left\langle u^{\prime}\right\rangle$ for some $u \neq u^{\prime}$. For this equivalence, the cases of protocol messages are similar to the previous equivalence, so we only detail the cases of test queries.

- When $Q_{1} \mid Q_{T}^{\prime}$ receives a message test $_{A}[i]\left(u_{A}\right), Q_{T A}^{\prime}$ first tests if $k_{A}\left[u_{A}\right]$ and $Y\left[u_{A}\right]$ are defined. Correspondingly, in the right-hand side, $Q_{S T A}$ first tests if $x_{r+1}^{\prime}\left[u_{A}\right]$ is defined and different from reject, which is equivalent to $k_{A}\left[u_{A}\right]$ and $Y\left[u_{A}\right]$ defined.

Next, if $Y\left[u_{A}\right] \neq B$, then $Q_{1} \mid Q_{T}^{\prime}$ sends $k_{A}\left[u_{A}\right]$ on $\operatorname{test}_{A}[i]$. Correspondingly, in the right hand-side, if $y_{r+1}^{\prime}\left[u_{A}\right] \neq \operatorname{accept}_{A}(B)$, that is, $Y\left[u_{A}\right] \neq B$, then $Q_{S T A}$ sends a message on $c_{A K}^{\prime}\left[u_{A}\right] . Q_{0}^{\prime}$ receives it, and replies by sending $k_{A}\left[u_{A}\right]$ on $c_{A K}^{\prime}\left[u_{A}\right]$. $Q_{S T A}$ receives this message, and sends $k=k_{A}\left[u_{A}\right]$ on $\operatorname{test}_{A}[i]$, as in the left-hand side.

Otherwise, both sides execute two finds that yield the same result because $\widetilde{x}\left[u_{A}\right]=\widetilde{x^{\prime}}\left[u_{A}\right], \widetilde{y}\left[u_{B}\right]=\widetilde{y^{\prime}}\left[u_{B}\right]$, and as we shall see below $r_{A}[u]$ and $r_{B}[u]$ have the same value in both sides of the equivalence.
Finally, when both finds fail, in the left-hand side, $Q_{T A}^{\prime}$ sends a fresh random number uniformly distributed in $I_{\eta}(T)$ on test $_{A}[i]$. Correspondingly, in the right-hand side, $Q_{S T A}$ sends $u_{A}$ on $c^{\prime}[i] . Q_{k_{A}^{\prime}}^{\prime}$ receives this message. It checks that $k_{A}^{\prime}\left[u_{A}\right]$ is defined, which is true because $k_{A}\left[u_{A}\right]$ is defined and $Y\left[u_{A}\right]=B$. Next, it looks for a previous query with the same $u_{A}$; there is no such query, because otherwise one of the previous finds would have succeeded:

- If $u_{A}$ was previously sent on $c^{\prime}\left[i^{\prime}\right]$ by $Q_{S T A}$, then there would be an $u\left(u=i^{\prime}\right)$ such that $u_{A}[u]$ and $r_{A}[u]$ are defined and $u_{A}[u]=u_{A}$, so the first find would have succeeded.

- If $u_{A}$ was previously sent on $c^{\prime}\left[i^{\prime}+n_{T}\right]$ by $Q_{S T B}$, then there would be an $u\left(u=i^{\prime}\right)$ such that $u_{A}^{\prime}[u]=u_{A}, \operatorname{sid}\left(\widetilde{x^{\prime}}\left[u_{A}^{\prime}[u]\right]\right)=\operatorname{sid}\left(\widetilde{y^{\prime}}\left[u_{B}[u]\right]\right)$, and these values are defined, so the second find would have succeeded.

So $Q_{k_{A}^{\prime}}^{\prime}$ replies by sending a fresh random number uniformly distributed in $I_{\eta}(T)$ on $c^{\prime}[i]$. $Q_{S T A}$ receives it, stores it in $r_{A}[i]$, and sends it on $\operatorname{test}_{A}[i]$, as in the left-hand side.

- When $Q_{1} \mid Q_{T}$ receives a message test $_{B}[i]\left(u_{B}\right)$, the situation is almost symmetric of the previous case. We only detail the case in which $y_{r+1}^{\prime}\left[u_{B}\right]=\operatorname{accept}_{B}(A)$ and the first two finds of $Q_{T B}^{\prime}$ and $Q_{S T B}$ fail. In this case, in the left-hand side, $Q_{T B}^{\prime}$ sends a fresh random number uniformly distributed in $I_{\eta}(T)$ on test ${ }_{B}[i]$. In the right-hand side, as in the proof of the previous equivalence, the last find of $Q_{S T B}$ succeeds, $\operatorname{sid}\left(\widetilde{x^{\prime}}\left[u_{A}^{\prime}\right]\right)=\operatorname{sid}\left(\widetilde{y^{\prime}}\left[u_{B}\right]\right), x_{r+1}^{\prime}\left[u_{A}^{\prime}\right]=\operatorname{accept}_{A}(B)$, $k_{A}\left[u_{A}^{\prime}\right]$ is defined, and $Y\left[u_{A}^{\prime}\right]=B$. So $Q_{S T B}$ sends $u_{A}^{\prime}$ on $c^{\prime}\left[i+n_{T}\right]$. $Q_{k_{A}^{\prime}}^{\prime}$ receives this message. It checks that $k_{A}^{\prime}\left[u_{A}^{\prime}\right]$ is defined, which is true because $k_{A}\left[u_{A}^{\prime}\right]$ is defined and $Y\left[u_{A}^{\prime}\right]=B$. Next, it looks for a previous query with the same $u_{A}^{\prime}$; there is no such query, because otherwise one of the previous finds would have succeeded:

- If $u_{A}^{\prime}$ was previously sent on $c^{\prime}\left[i^{\prime}+n_{T}\right]$ by $Q_{S T B}$, then there would be an $u\left(u=i^{\prime}\right)$ such that $u_{A}^{\prime}[u]$ and $r_{B}[u]$ are defined and $u_{A}^{\prime}[u]=u_{A}^{\prime}$. Then $u_{B}[u]$ is also defined, $\operatorname{sid}\left(\widetilde{x^{\prime}}\left[u_{A}^{\prime}[u]\right]\right)=$ $\operatorname{sid}\left(\widetilde{y^{\prime}}\left[u_{B}[u]\right]\right), x_{r+1}^{\prime}\left[u_{A}^{\prime}[u]\right]=\operatorname{accept}_{A}(B)$. So we have $\operatorname{sid}\left(\widetilde{y}\left[u_{B}[u]\right]\right)=\operatorname{sid}\left(\widetilde{y^{\prime}}\left[u_{B}[u]\right]\right)=$ $\operatorname{sid}\left(\widetilde{x^{\prime}}\left[u_{A}^{\prime}[u]\right]\right)=\operatorname{sid}\left(\widetilde{x^{\prime}}\left[u_{A}^{\prime}\right]\right)=\operatorname{sid}\left(\widetilde{y^{\prime}}\left[u_{B}\right]\right)=$ $\operatorname{sid}\left(\widetilde{y}\left[u_{B}\right]\right)$. In order to obtain a contradiction, assume that $u_{B}[u] \neq u_{B}$. The event $\operatorname{full}_{B}(A$, $\left.k_{B}\left[u_{B}[u]\right], \operatorname{sid}\left(\widetilde{y}\left[u_{B}\right]\right)\right)$ has been executed in copy number $u_{B}[u]$ of $Q_{B}$ and $f_{u l l}\left(A, k_{B}\left[u_{B}\right]\right.$, 
$\left.\operatorname{sid}\left(\widetilde{y}\left[u_{B}\right]\right)\right)$ has been executed in copy number $u_{B}$ of $Q_{B}$. Since the correspondence (6) is injective, two distinct events $\operatorname{full}_{A}\left(B, k_{B}\left[u_{B}[u]\right]\right.$, $\left.\operatorname{sid}\left(\widetilde{y}\left[u_{B}\right]\right)\right)$ and $\operatorname{full}_{A}\left(B, k_{B}\left[u_{B}\right], \operatorname{sid}\left(\widetilde{y}\left[u_{B}\right]\right)\right)$ have been executed. So $k_{B}\left[u_{B}[u]\right]=k_{A}\left[u_{A 1}\right]$ and $k_{B}\left[u_{B}\right]=k_{A}\left[u_{A 2}\right]$ with $u_{A 1} \neq u_{A 2}$. Moreover, by the correspondence (7), since the events $\operatorname{full}_{B}\left(A, k_{B}\left[u_{B}[u]\right], \operatorname{sid}\left(\widetilde{y}\left[u_{B}\right]\right)\right)$ and $\operatorname{full}_{A}\left(B, k_{B}\left[u_{B}\right], \operatorname{sid}\left(\widetilde{y}\left[u_{B}\right]\right)\right)$ have been executed, $k_{B}\left[u_{B}[u]\right]=k_{B}\left[u_{B}\right]$, so $k_{A}\left[u_{A 1}\right]=$ $k_{A}\left[u_{A 2}\right]$ with $u_{A 1} \neq u_{A 2}$. This contradicts the exclusion of traces with $k_{A}[u]=k_{A}\left[u^{\prime}\right]$ for some $u \neq u^{\prime}$. So $u_{B}[u]=u_{B} .^{2}$ So the first find would have succeeded.

- If $u_{A}^{\prime}$ was previously sent on $c^{\prime}\left[i^{\prime}\right]$ by $Q_{S T A}$, then there would be an $u\left(u=i^{\prime}\right)$ such that $u_{A}[u]$ and $r_{A}[u]$ are defined and $u_{A}[u]=u_{A}^{\prime}$. Since the last find of $Q_{S T B}$ succeeds, $\operatorname{sid}\left(\widetilde{x^{\prime}}\left[u_{A}^{\prime}\right]\right)=$ $\operatorname{sid}\left(\widetilde{y^{\prime}}\left[u_{B}\right]\right), \operatorname{sos} \operatorname{sid}\left(\widetilde{x^{\prime}}\left[u_{A}[u]\right]\right)=\operatorname{sid}\left(\widetilde{y^{\prime}}\left[u_{B}\right]\right)$, so the second find would have succeeded.

So $Q_{k_{A}^{\prime}}^{\prime}$ replies by sending a fresh random number uniformly distributed in $I_{\eta}(T)$ on $c^{\prime}\left[i+n_{T}\right]$. $Q_{S T B}$ receives it, stores it in $r_{B}[i]$, and sends it on test $_{B}[i]$, as in the left-hand side.

\section{Discussion on Authentication and Key Ex- change}

We discuss here some choices made in our modeling of authentication and key exchange.

- We have assumed that $A$ plays only the role of the initiator and $B$ plays only the role of the responder. We could also model a situation in which $A$ and $B$ play both roles, by including a process $Q_{A}^{\prime}$ for $A$ playing the responder role and a process $Q_{B}^{\prime}$ for $B$ playing the initiator role. Which model is more appropriate depends on the protocol and its intended usage: the former model is appropriate for protocols that use distinct keys for the initiator and responder roles, such as SSH for instance.

- We could also extend the framework to protocols that use a trusted server, by including it into $Q_{S}$.

- For simplicity, we have assumed that the participants terminate immediately after accepting; we could obviously extend the framework to allow them to accept before the end of the protocol.

\footnotetext{
${ }^{2}$ More generally, if $\operatorname{sid}\left(\widetilde{y}\left[u_{B}^{\prime}\right]\right)=\operatorname{sid}\left(\widetilde{y}\left[u_{B}\right]\right)$, then $u_{B}^{\prime}=u_{B}$. So two sessions can have the same session identifiers only with negligible probability.
}

- [16] uses the notion of matching conversations instead of sessions identifiers. Matching conversations correspond to session identifiers when $\operatorname{sid}\left(x_{1}, \ldots\right.$, $\left.x_{r}\right)=\left(x_{1}, \ldots, x_{r}\right)$ and $\operatorname{sid}^{\prime}\left(x_{1}, \ldots, x_{r-1}\right)=\left(x_{1}\right.$, $\left.\ldots, x_{r-1}\right)$ with the additional requirement that the messages from $A$ to $B$ are received by $B$ after they are sent by $A$ and symmetrically. We do not consider this requirement here, because it would complicate the verification considerably. We partly compensate for this weaker definition by checking an injective correspondence, while [16] infers injectivity from the correct ordering of messages - see [16, Appendix C]. More recent formalizations $[7,15,27,40,42]$ use session identifiers as we do.

- It is often required that, with overwhelming probability, distinct sessions have distinct session identifiers. Here, we only require that $n$ sessions of $A$ with the same identifier correspond to $n$ sessions of $B$ with that identifier. For authenticated key exchange, the secrecy of the key combined with the correspondence (7) (which means that two sessions with same identifier have the same key) implies that, with overwhelming probability, distinct sessions have distinct session identifiers.

\section{E. Detailed Experimental Results}

In our tests, all protocols are in a configuration in which the honest participants are willing to run sessions with the adversary. Shared-key encryption is implemented as encrypt-then-MAC, where the encryption is IND-CPA (indistinguishability under chosen plaintext attacks) and the MAC is UF-CMA (unforgeability under chosen message attacks); public-key encryption is assumed to be IND-CCA2 (indistinguishability under adaptive chosen ciphertext attacks); signatures are assumed to be UF-CMA.

The session identifier is chosen to contain all messages of the protocol, except messages that are sent to or received from a server (that is, messages that are not between $A$ and $B$ ), messages that are just forwarded without checking (those can be changed by the adversary), and signatures when the security definition of signatures allows an adversary to forge a new signature for a message that has already been signed.

For the public key protocols, the prover needs to be given the main proof steps. We detail them below. For shared-key protocols, the proof is fully automatic.

Woo-Lam shared-key [36] This protocol is a one-way authentication protocol, so we prove only the correspondence (4). Our prover cannot prove this correspondence for 
the original version of the protocol, as there is a known attack against it, but proves it for the corrected version [36].

Woo-Lam public-key [62] The situation is similar to the Woo-Lam shared key protocol. Our prover cannot prove the correspondence (4) for the original version of the protocol, as there is an attack against it, but proves it for the corrected version [64].

In this protocol, the third message is a signature. The proof fails when the signature is included in the session identifier and the security definition of signatures allows an adversary to forge a new signature for a message that has already been signed. Indeed, the signature is not authenticated in this case. The proof succeeds both when the signature is not included in the session identifier and when the security definition of signatures prevents forgeries even for already signed messages, that is, signatures are SUF-CMA (strongly unforgeable under chosen-message attacks).

For both versions of this protocol, we give the following proof steps to the prover:

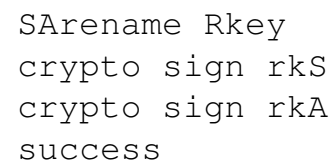

The variable Rkey defines a table of public keys, and is assigned at three places, corresponding to principals $A$ and $B$, and to other principals defined by the adversary. The transformation SArename Rkey renames the variable Rkey to three different names Rkey1, Rkey2, and Rkey 3 , one for each assignment to Rkey, and thus allows us to distinguish these three cases. The instruction crypto sign $r k S$ means that the prover should apply the definition of security of signatures (primitive sign), for the key generated from random number $r k S$. The instruction success means that prover should check whether the desired security properties are proved.

Needham-Schroeder public-key [53] This protocol is a mutual authentication protocol. Our prover shows the correspondence (3) but the proof fails for (4); indeed, there is a well-known attack against it [48]. The prover proves both (3) and (4) for the corrected version [48].

For both versions of this protocol, we give the following proof steps to the prover:

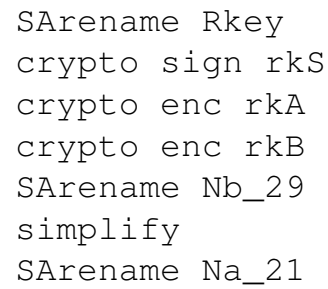

simplify

success

Denning-Sacco public-key [34] This protocol is a key exchange protocol, so we try to prove the hypothesis of Proposition 4. Since there is no message from $B$ to $A$ in this protocol, $B$ is not authenticated to $A$, so (5) clearly does not hold. (There is in fact no good place for putting the event $\left.\operatorname{part}_{B}.\right)$ For both the original and the corrected version of [5], this protocol is also subject to an obvious replay attack, so unsurprisingly our prover cannot show the injective correspondence (6). Our prover shows (7) for both the original and the corrected version. It shows the secrecy of $k_{A}^{\prime}$ and the non-injective correspondence event $\left(f u l l_{B}(A, k, x)\right) \Rightarrow \operatorname{event}\left(f u l l_{A}(B, k, x)\right)$ only for the corrected version. (There is a well-known attack [5] against them in the original version.)

For both versions of this protocol, we give the following proof steps to the prover:

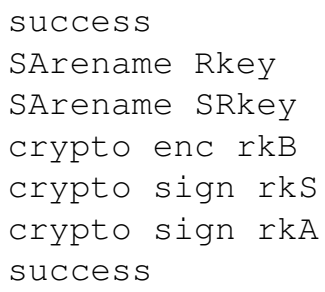

The first success instruction is useful in order to prove (7): this correspondence is obvious on the initial game, because the key $k$ or $k^{\prime}$ is computed from the protocol messages contained in the session identifier $x$. The relation between the key $k$ and the session identifier $x$ is hidden by the subsequent game transformations.

Needham-Schroeder shared-key [53] The proof of secrecy of the key fails for both the original and the corrected version [54]: the protocol contains a key confirmation round $B \rightarrow A:\left\{N_{B}\right\}_{K}, A \rightarrow B:\left\{N_{B}-1\right\}_{K}$ and these messages may reveal information on the key $K$. However, the prover shows (3) but fails to show (4) for the original version of the protocol. This failure comes from a limitation of our prover: it fails to prove that $N_{B}[i] \neq N_{B}\left[i^{\prime}\right]-1$ with overwhelming probability, where $N_{B}$ is a nonce. (Proving this property requires distinguishing two cases: when $i=i^{\prime}$, we have $N_{B}[i] \neq N_{B}[i]-1$; when $i \neq i^{\prime}$, both sides are independent random numbers, which have a negligible probability of being equal. ${ }^{3}$ ) The prover shows both (3) and (4) for the corrected version. When the key confirmation round is removed, the prover proves the secrecy of the key $k_{A}^{\prime}$, but fails to prove the authentication (which is indeed wrong).

\footnotetext{
${ }^{3}$ This issue has been solved in CryptoVerif 1.20 , so that CryptoVerif now succeeds in proving (4).
} 
Yahalom [23] The situation is similar to the NeedhamSchroeder shared-key protocol: the proof of secrecy of the key fails because of a key confirmation message $\left\{N_{B}\right\}_{K}$. The prover still shows (3) and (4). When the key confirmation message is removed, the prover shows (3) but fails to show (4) (which is indeed wrong).

Otway-Rees [55] The prover shows the secrecy of $k_{A}^{\prime}$, but does not show the correspondence properties (5), (6), and (7). These correspondences are indeed wrong: as noticed in [23], each participant may accept while the other participant fails to get the key, so (6) is wrong. The correspondences (5) and (7) are wrong due to replay attacks. 